\title{
Endogenous insurance and informal relationships
}

\author{
Xiao Yu Wang*
}

February 23, 2015

\begin{abstract}
Heterogeneously risk-averse individuals who lack access to formal insurance build and use relationships with each other to manage risk. I show that the composition of equilibrium relationships under pairwise matching and when group size is endogenous is determined by a mean-variance trade-off across differentially risky productive opportunities, though output distributions may have infinitely-many nonzero cumulants. This has important policy implications. For example, a policy which ignores the equilibrium response of informal institutions may exacerbate inequality and hurt most those it intended to help: a reduction in aggregate risk may lead to an increase in risk borne by the most risk-averse individuals, as the least risk-averse abandon their roles as informal insurers. The theory also sheds light on the channels through which endogenous insurance relationships influence informal firm structure and entrepreneurship.
\end{abstract}

\section{Introduction}

This paper develops a theory of the equilibrium formation and structure of the relationships which poor, risk-averse people build with each other, when they lack access to formal risk management tools. The risk-sharing literature has focused largely on analyzing the insurance agreement reached by a fixed, isolated group of individuals, or by individuals who match with a fixed probability, and has focused less on what groups might actually exist and coexist in the first place. Endogenizing the structure of informal insurance improves policy analysis by enabling a more thorough understanding of how people re-optimize in response to policies, and yields insights into the connections between informal insurance and income inequality, entrepreneurship, and the structure of informal firms.

\footnotetext{
*Department of Economics, Duke University, 213 Social Sciences, Box 90097, Durham NC 27708. Email: xy.wang@duke.edu. I am indebted to Abhijit Banerjee, Esther Duflo, and Rob Townsend for their insights, guidance, and support. I thank Attila Ambrus, Dan Bennett, Gabriel Carroll, Arun Chandrasekhar, Sebastian Di Tella, Erica Field, Ben Golub, Rachel Kranton, Andy Newman, Debraj Ray, Chris Walters, Juan Pablo Xandri, and seminar and conference participants at the CEAR Household and Risk Symposium, Chicago, Duke, NEUDC at Dartmouth, Notre Dame, Stanford, ThReD at Oslo, Yale, Warwick, and the World Bank for helpful comments. Any errors are my own. Support from the National Science Foundation is gratefully acknowledged.
} 
I develop a model inspired by a lively informal insurance literature. ${ }^{1}$ Risk imposes a serious burden on the poor, and the desire to manage risk evidently influences many dimensions of the lives of poor individuals. For example, Rosenzweig and Stark (1989) show that daughters of more riskaverse farmers are married to more distant villages, to minimize the correlation between farming incomes. Stiglitz (1974) shows that sharecropping arises when landowners layer insurance provision on top of incentives for tenant farmers, and Ackerberg and Botticini (2002) find evidence that heterogeneously risk-averse tenant farmers and landlords in medieval Tuscany strategically formed sharecropping relationships based on differing risk attitudes. Thus, the poor use complex relationships with each other in a variety of creative ways when they lack access to formal insurance and credit institutions.

The model has the following key elements. Risk-averse individuals with exponential utility work together to be productive. For example, in an agricultural village, some individuals own land but would prefer not to farm it themselves, while other, landless individuals have both the willingness and the skill to farm. Alternatively, an investor and an entrepreneur, or two entrepreneurs with different sets of skills and resources, must work together to launch a business. Matching is assumed to be pairwise in the benchmark model, but this is later relaxed to allow group size itself to be endogenous.

There are two key types of heterogeneity: heterogeneity of preferences, and heterogeneity of technology. Individuals vary in their degree of constant absolute risk aversion, and a matched group chooses a joint income distribution from a set of differentially risky options, or "projects". (This is substantively equivalent to the case where individuals each choose an income distribution and share the pooled realizations. ${ }^{2}$ ) Projects with higher expected return come at the cost of higher variance of return, and members of a group share the realized return of their chosen project according to a rule determined ex ante. For example, investors seeking to form joint ventures are presented with business proposals of differing riskiness, and a landowner and her tenant farmer face a spectrum of portfolios of crops, land plots, inputs, and farming methods, which each yield different profit distributions. I allow for a large class of symmetric and skewed return distributions. Importantly, distributions may have infinitely many higher order cumulants (as opposed to just two nonzero cumulants, as in the case of the normal distribution). ${ }^{3}$

The results reveal that accounting for the endogeneity of informal institutions can be essential for policymaking. I show that a policy which reduces aggregate risk is a strict Pareto improvement

\footnotetext{
${ }^{1}$ For background and institutional details, see, for example, Alderman and Paxson (1992), Dercon (2004), Fafchamps (2008, and Morduch (1995). Empirical risk-sharing papers include Dercon and Krishnan (2000), Fafchamps and Lund (2003), Mazzocco and Saini (2012), and Townsend (1994). Theoretical papers on informal insurance include Bloch et. al. (2008), Genicot and Ray (2003), and Ligon et. al. (2002). Theoretical and empirical papers on risk-sharing networks include Ambrus et al. (2013), Bramoulle and Kranton (2007), and Fafchamps and Gubert (2007).

${ }^{2} \mathrm{~A}$ simplified intuition for the equivalence is that even if individuals choose their own income distributions, the matching is driven by individuals' anticipation of what their joint income distribution would be with different partners. Thus, matched individuals are effectively jointly choosing a distribution of pooled income. Please see Appendix 1 for more details.

${ }^{3}$ Recall that the cumulant-generating function is the log of the moment-generating function.
} 
if informal insurance is assumed to stay fixed. However, accounting for the endogenous network response may change this analysis entirely. Those the policy is particularly intended to help, the most risk-averse, are in fact particularly harmed: the change in the risk environment incentivizes the less risk-averse agents to abandon their roles as informal insurers of the more risk-averse, in favor of partnerships with other less risk-averse agents. This forces the most risk-averse agents to match with each other instead, leaving them strictly worse off through two channels. First, they've lost insurance through weakened consumption-smoothing-each member wants her consumption to depend as little as possible on her income realization, but neither partner is willing to absorb the volatility to provide this. This is compounded for these more risk-averse agents by an additional channel: the loss of consumption-smoothing means that the group must rely on income-smoothing to manage risk. But this means selecting projects with particularly low expected return, so that the more risk-averse agents are unable to take advantage of a policy which reduces the variance of the higher expected return, entrepreneurial projects.

Notably, this is the case despite the ability of individuals to commit ex ante to a returncontingent sharing rule. This is an interesting addition to several papers which study the symbiosis of formal and informal insurance. Attanasio and Rios-Rull (2000) model informal insurance as being fundamentally about limited commitment, where members of an exogenously-given group punish those who renege by consigning them to autarky. They find that aggregate risk reduction resulting from the strengthening of formal insurance may decrease welfare by making autarky more appealing, thereby lessening the punishment for reneging. My results illustrate that accounting for the re-optimization of relationships may be just as important for understanding informal insurance as accounting for a weaker contracting environment. ${ }^{4}$ Mobarak and Rosenzweig (2012) study a different interaction between formal and informal insurance. They find that formal insurance, which comes with basis risk, acts as a complement to informal insurance when informal methods manage idiosyncratic risk well, and acts as a substitute when they don't. However, the strength of informal insurance is fixed exogenously. By endogenizing informal insurance, this paper raises a variety of interesting ways to build on these findings-for example, this paper ties an understanding of when informal insurance is relatively strong or weak to data-based conditions, suggesting an approach for policymakers to know when to expect formal insurance to act as a complement or a substitute.

The approach to deriving these results is summarized as follows. First, I find a transferable utility representation of the model. Since agents are heterogeneous in risk attitudes, a single unit of output generates a different level of utility for one agent than it does for another-thus, utility is non-transferable. However, I show that expected utility (which can be represented by the certaintyequivalent) is transferable, and seek conditions under which the total certainty-equivalent of a matched group exhibits supermodularity and submodularity in risk attitudes. This approach is inspired by Schulhofer-Wohl (2006) and Legros and Newman (2007), who both observe that models

\footnotetext{
${ }^{4}$ Although the intent of the model is to focus on the endogeneity of informal insurance and shut down commitment problems, I discuss the effect of introducing limited commitment in Appendix 12.
} 
with non-transferable utility may admit a transferable utility representation. ${ }^{5}$ Schulhofer-Wohl identifies conditions on preferences under which expected utility is transferable in a more basic risk-sharing problem, where agents face an exogenous risk and can commit ex ante to a returncontingent sharing rule, but are not able to choose what risk they face.

The key observation is that the total certainty-equivalent of a matched group is the product of the cumulant-generating function of the return distribution of the group's chosen project, and the group's representative risk tolerance. I show that, although the return distributions may have infinitely many nonzero cumulants, unique assortative matching in risk attitude is determined by the first two cumulants alone (the mean and the variance), and does not depend on any aspect of the distribution of risk attitudes in the population. Specifically, if the coefficient of variation of return (the ratio of the standard deviation to the mean) across all possible projects is decreasing in the mean (that is, projects with higher expected return have a smaller coefficient of variation), then unique positive assortative matching results in the pairwise equilibrium, while if the coefficient of variation is increasing in the mean, unique negative assortative matching results in the pairwise equilibrium. Note that the preference structure is not driving this result-for example, the entire sequence of cumulants matters when the set of return distributions does not satisfy the properties described in the model. ${ }^{6}$

But when is the coefficient of variation decreasing or increasing in expected return? Think of the function describing the variance of project return when the mean return is $p$ as the "cost" of obtaining an expected return $p$. Then the coefficient of variation is decreasing (increasing) in mean return if and only if the marginal cost function is concave (convex) in the mean $p$. Intuitively, equilibrium matching is driven by the trade-off between preference for a similar partner when choosing risk ex ante, and preference for a dissimilar partner when sharing risk ex post, and the curvature of the marginal cost function captures this trade-off. I show that when the ability to share risk ex post is shut down, e.g. because the government passes a wage law which effectively fixes sharing rules, positive assortative matching is always the unique equilibrium. This is supported by the experimental finding of Attanasio et al. (2012), who find that individuals match positive assortatively in risk type when they know each other's types, and when they are able to choose what risky gamble to face, but must equally share returns. By contrast, when the ability to choose risk ex ante is shut down, negative assortative matching is always the unique equilibrium. This coincides with the theoretical finding of Legros and Newman (2007), Schulhofer-Wohl (2006), and Chiappori and Reny (2006), and is supported by the empirical finding discussed earlier of Ackerberg and Botticini (2002) that landowners and farmers matched negative assortatively.

Finally, I allow group size itself to be endogenous. Using coalitional stability as the equilibrium criterion, I show that, under the condition yielding unique positive assortative matching in the pairwise case, whole-group matching (maximal connectedness) is the unique equilibrium, while

\footnotetext{
${ }^{5}$ Legros and Newman (2007) develop a more general method to characterize conditions for assortative matching in settings where utility is not transferable.

${ }^{6}$ See Appendix 9 for more detail.

Note that the reciprocal of the coefficient of variation is known as the Sharpe ratio (Sharpe (1966).
} 
under the condition yielding unique negative assortative matching in the pairwise case, negative assortative, pairwise matching (minimal connectedness) continues to be the unique equilibrium. Thus, extremal network shapes are tied to extremal within-group composition, which yields intriguing predictions for the structure of informal firms. The result provides an interesting contrast to Genicot and Ray (2003), who find that, under limited commitment, whole-group matching can never be sustained. In their model, because individuals cannot choose what risk they face and cannot commit to a sharing rule, the marginal benefit of an additional member is eventually negative: an individual's expected income is independent of group size, but she becomes increasingly likely to bear a partner's downside risk as the group grows. In my model, it is possible for the marginal benefit of an additional member to be positive even for arbitrarily large groups, since the trade-off is slightly different: adding a member allows the group to take on additional risk because of the increased ability to share risk ex post, which the group can leverage into taking up a higher mean, higher variance project. However, now the realized surplus must be divided across more people. I show that the curvature of the marginal variance cost function again determines which force outweighs.

In the next section, I set up the benchmark model with pairwise matching and provide the main matching results. I then study a hypothetical policy, show how it maps into the theoretical framework, and demonstrate that the evaluation of the welfare impact changes dramatically after accounting for the response of the network. Following this, I extend the model and allow group size itself to be endogenous. Then, I discuss falsifiability of the theory, and show support for the theory in the existing empirical literature. Finally, I conclude. Technical details are relegated to the Appendix (A).

\section{The Model}

In the benchmark model, matching is restricted to be pairwise. In Section 5, I relax the constraint of partnerships and analyze this model when group size itself is endogenous.

\subsection{Setup}

The population of agents: the economy is populated by two groups of agents, $G 1$ and $G 2$, where $|G 1|=|G 2|=Z, Z \in\{2,3,4, \ldots\} .^{7}$ (The case $|G 1| \neq|G 2|$ does not substantively change the results: the most risk-averse individuals of the larger group simply remain unmatched. See Appendix 3 for details.) Agents differ in their Arrow-Pratt degree of absolute risk aversion $r$, where an agent $i$ of type $r_{i}$ receives utility $u_{i}(x)=-e^{-r_{i} x}$ from consuming $x$ units of output. Let $r_{i}>0$ : each individual is risk-averse to some extent. Define agent $r_{i}$ 's degree of risk tolerance: $R_{i} \equiv \frac{1}{r_{i}}$.

\footnotetext{
${ }^{7}$ Matching is assumed to be across two groups rather than within a single group because assortative matching patterns are well-defined under the former, not because it is necessary for the results. To see this, suppose that matching is within a group of four people, $\left\{r_{1}, r_{2}, r_{3}, r_{4}\right\}$, ordered from least to most risk-averse. Then there are two possible positive assortative matchings: $\left\{\left(r_{1}, r_{2}\right),\left(r_{3}, r_{4}\right)\right\}$, and $\left\{\left(r_{1}, r_{3}\right),\left(r_{2}, r_{4}\right)\right\}$.
} 
No assumptions are imposed on the distributions of risk preferences within each group, or across groups.

The risky environment: a spectrum of risky projects is available, with return distributions parameterized by $p \in \Pi \subseteq \mathbb{R}_{0}^{+}$. A project $p$ returns $Y_{p}$, a random variable described by:

$$
Y_{p}=p+V(p)^{\frac{1}{2}} Y
$$

where $Y$ is a random variable with a well-defined cdf $F_{Y}: \mathbb{R} \rightarrow[0,1]$, and $E(Y)=0, V(Y)=1$. Note that this allows for a large class of possible distributions for project returns, which may be symmetric or skewed, including the Normal, Laplace, Logistic, Student, and generalized extreme value (e.g. Gumbel) distributions. ${ }^{8}$ (Appendix 10 re-expresses this portfolio choice problem as a decision between investing in a risky and a risk-free asset, for readers who find that formulation more intuitive. Section 4 provides a concrete example of how farmers choosing between crop portfolios with varying profit distributions can be represented in this way.)

Hence:

$$
\begin{aligned}
E\left(Y_{p}\right) & =p \\
V\left(Y_{p}\right) & =V(p)
\end{aligned}
$$

The function $V: \Pi \rightarrow \mathbb{R}_{0}^{+}$describes the variance of a project with expected return $p$. Assume that $\Pi=\mathbb{R}_{0}^{+}$, so that there exists a portfolio which is not strictly dominated achieving expected return $p$ for each $p \geq 0$.

Let $V(\cdot)$ be thrice-differentiable, and:

1. $V(0)=0, V(p)>0$ for $p>0$.

2. $V^{\prime}(0)=0, V^{\prime}(p)>0$ for $p>0$.

3. $V^{\prime \prime}(p)>0$.

The first property ensures that variance is nonnegative, and that an action which returns 0 with certainty exists (e.g. "do nothing") ${ }^{9}$. The second property ensures that projects with higher mean return also have higher variance of return (Appendix 10 discusses the portfolio choice problem in detail and describes conditions under which this arises). Finally, the third property ensures an interior solution for project choice for any agent $r$.

A subset of the risky projects available might therefore be represented like this:

\footnotetext{
${ }^{8} \mathrm{~A}$ family of distributions with this property is sometimes called a location-scale family.

${ }^{9}$ Imagine instead that the variance of a project with mean $p$ were given by $V(p)+c$, where $V(0)=0, c>0$. Then the level $c$ affects what an individual's project choice is, but does not affect the marginal variance cost across projects, which is what's crucial for matching. Thus, I simply assume $c=0$, which has the effect of a normalization.
} 


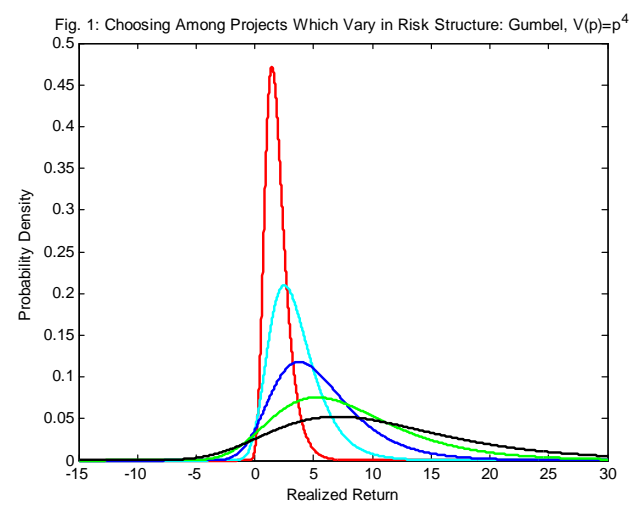

Production: Assume that any project $p$ requires the partnership of two agents, one from $G 1$ and one from G2. For example, a landowner and a tenant farmer must work together to choose inputs and grow crops, two adults marry to form a productive household, an investor funds an entrepreneur with a business proposal, two entrepreneurs with different skill sets and time constraints work together to form a successful joint venture, and so on. A matched pair $\left(r_{1}, r_{2}\right)$ jointly selects a project. (See Appendix 1 for a proof that this is equivalent to one where each partner individually chooses a project and both share the pooled returns.)

All matched pairs face the same spectrum of projects, each agent can be involved in at most one project, and there are no "project externalities". That is, one pair's project choice does not affect availability or returns of any other pair's project.

To focus on the impact on equilibrium matching of the trade-off in ex ante and ex post risk management across partnerships of different risk compositions, there is no moral hazard in this model, although it is straightforward to allow for observable, contractible actions. Please refer to Wang (2013b) for an explicit treatment of moral hazard and informal insurance in an endogenous matching problem.

Information and commitment: all agents know each other's risk types and the risk environment.

A given matched pair $\left(r_{1}, r_{2}\right)$ undertaking project $p_{12}$ observes the realized output $y_{p_{12}}$ of their partnership, and is able to commit ex ante to a feasible return-contingent sharing rule $s: \mathbb{R} \rightarrow \mathbb{R}$ (there is no limited liability). Denote $r_{2}$ 's share of realized output by $s\left(y_{p_{12}}\right)$. Feasibility implies that the income $r_{1}$ receives must be less than or equal to $y_{p_{12}}-s\left(y_{p_{12}}\right)$. Since all agents have monotonically increasing utility, $r_{1}$ 's share will be equal to $y_{p_{12}}-s\left(y_{p_{12}}\right)$.

The equilibrium: An equilibrium is ${ }^{10}$ :

1. The matching pattern: a match function $\mu: \mathbb{R}^{+} \rightarrow \mathbb{R}^{+}$, mapping each agent in group 1 to a single agent in group 2. Thus, $r_{1}$ 's partner is denoted by $\mu\left(r_{1}\right)$, and $\mu(\cdot)$ assigns distinct members of group 1 to distinct partners in group $2 .^{11}$

\footnotetext{
${ }^{10}$ Existence is assured by Kaneko (1982).

${ }^{11}$ In practice, characteristics other than risk aversion, such as kinship and friendship ties, surely factor into matching
} 
Moreover, the matching pattern described by $\mu(\cdot)$ must be stable. It must be that no agent is able to propose a feasible project and sharing rule to an agent not matched to her under $\mu$, such that both agents are happier when matched with each other in this proposed arrangement than they are with the partners assigned by $\mu$ ("no blocks"). ${ }^{12}$

2. The risky projects: a project choice for each matched pair, such that no pair can achieve weakly better outcomes for both partners (and a strictly better outcome for at least one partner) by choosing a different project. In other words, the project chosen by a matched pair must be optimal for that pair.

3. Individual payoffs and sharing rules: a sharing rule for each matched pair, describing the amount each partner receives given each possible return realization, where the sum of shares cannot exceed the total return (feasibility). The sharing rule must be such that no pair can achieve weakly better outcomes for both partners (and a strictly better outcome for at least one partner) by choosing a different sharing rule. In other words, the sharing rule chosen by a matched pair must be optimal for that pair.

Individual payoffs will not be unique in the equilibrium of this model. Instead, the stability conditions will determine a set of equilibrium surplus divisions. For a matched pair $\left(r_{i}, \mu\left(r_{i}\right)\right)$, let $v_{i}$ denote the expected utility of $\mu\left(r_{i}\right)$ in equilibrium, and $\phi\left(r_{i}, \mu\left(r_{i}\right), v_{i}\right)$ denote $r_{i}$ 's maximal expected utility given that $\mu\left(r_{i}\right)$ 's expected utility is $v_{i}$. Then a vector of individual payoffs described by $\left(v_{1}, \ldots, v_{N}\right)$ can be supported in equilibrium if and only if for each $r_{i}$ :

$$
\phi\left(r_{i}, \mu\left(r_{i}\right), v_{i}\right) \geq \phi\left(r_{i}, \mu\left(r_{j}\right), v_{i}\right) \forall j \neq i
$$

That is, given the divison of surplus described by $\left(v_{1}, \ldots, v_{N}\right)$, no two individuals who are unmatched are able to match with each other instead, and split the surplus they generate in such a way that they are both weakly better off, and at least one of them is strictly better off.

Matching patterns: Let $G_{j}=\left\{r_{j}^{1}, r_{j}^{2}, \ldots, r_{j}^{Z}\right\}, j \in\{1,2\}$, ordered from least to most riskaverse. Under "positive assortative matching" (PAM), the $i^{\text {th }}$ least risk-averse person in $G 1$ is matched with the $i^{\text {th }}$ least risk-averse person in $G 2: \mu\left(r_{1}^{i}\right)=r_{2}^{i}, i \in\{1, \ldots, Z\}$. For example, $\left(r_{1}^{1}, r_{2}^{1}\right)$ are matched under PAM.

On the other hand, under "negative assortative matching" (NAM), the $i^{\text {th }}$ least risk-averse person in $G 1$ is matched with the $i^{t h}$ most risk-averse person in $G 2: \mu\left(r_{1}^{i}\right)=r_{2}^{Z-i+1}, i \in\{1, \ldots, Z\}$. For example, $\left(r_{1}^{1}, r_{2}^{Z}\right)$ are matched under NAM.

decisions. However, the model is still key for understanding the equilibrium match. For example, friends and family are more likely to know each other's risk types, and are more likely to trust each other, and to monitor and discipline each other. Hence, an individual might first identify a pool of feasible risk-sharing partners, where this pool would be shaped by kinship and friendship ties, due to good information and commitment properties. Individuals would then choose their risk-sharing partners from these pools based on risk attitude.

${ }^{12}$ Individual rationality holds, as individuals cannot produce on their own. 
The unique equilibrium matching pattern is PAM (NAM) iff the only $\mu$ which is stable under optimal within-pair sharing rules and projects is the match function which assigns agents to each other positive (negative) assortatively in risk attitudes.

The next section discusses the results for this model, with technical details relegated to the Appendix.

\section{Results}

The first step is to identify a transferable utility representation for this model. (Schulhofer-Wohl (2006) identifies conditions on preferences under which expected utility is transferable in a more basic risk-sharing problem, where agents face an exogenous risk and can commit ex ante to a returncontingent sharing rule. That is, they are not able to choose what risk they face.) The heterogeneity of risk-aversion makes this a model of matching under nontransferable utility: one unit of output yields utility $u_{1}(1)=-e^{-r_{1}}$ for an agent with risk aversion $r_{1}$, but utility $u_{2}(1)=-e^{-r_{2}} \neq u_{1}(1)$ for an agent with risk aversion $r_{2}$. Thus, an individual evaluates potential partners based not only on how much output they can produce together, as in the standard case, but also on how happy the partner is with a given level of output-a partner who produces a lot but then demands a large transfer might be less desirable than a partner who produces less but is satisfied with little.

Lemma 1 Expected utility is transferable in this model. Denote a matched pair by $\left(r_{1}, r_{2}\right)$ and their chosen project by $p^{*}\left(r_{1}, r_{2}\right)$. Let $C E\left(r_{1}, r_{2}, p^{*}\left(r_{1}, r_{2}\right)\right)$ describe the certainty-equivalent of a matched pair in equilibrium; it is twice continuously differentiable in each argument. Then:

$$
\begin{aligned}
& \frac{\partial C E\left(r_{1}, r_{2}, p^{*}\left(r_{1}, r_{2}\right)\right)}{\partial r_{1} r_{2}}>0 \Leftrightarrow \text { unique PAM } \\
& \frac{\partial C E\left(r_{1}, r_{2}, p^{*}\left(r_{1}, r_{2}\right)\right)}{\partial r_{1} r_{2}}<0 \Leftrightarrow \text { unique } N A M
\end{aligned}
$$

The equilibrium matching maximizes the sum of certainty-equivalents, and is Pareto efficient.

A quick sketch of the proof of Lemma 1 provides a useful understanding of the matching problem, but all technical details for the proof of the lemma are relegated to Appendix 2.

First, we characterize the optimal project and sharing rule chosen by a matched pair $\left(r_{1}, r_{2}\right)$. Suppose $r_{1}$ and $r_{2}$ have already selected a project $p$. Let $v \in \mathbb{R}$ parameterize the division of surplus between the two partners. Then the program below characterizes the equilibrium sharing rule, given $v$. (Symmetry implies that the program could also have been set up fixing $r_{1}$ 's expected utility.)

$$
\begin{gathered}
\max _{s\left(y_{p}\right)} \int_{-\infty}^{\infty}-e^{-r_{1}\left[y_{p}-s\left(y_{p}\right)\right]} f\left(y_{p} \mid p\right) d y_{p} \quad \text { s.t. } \\
\int_{-\infty}^{\infty}-e^{-r_{2} s\left(y_{p}\right)} f\left(y_{p} \mid p\right) d y_{p} \geq-e^{-v}
\end{gathered}
$$


Solving this program shows that the optimal sharing rule is linear, where the more risk-averse partner's transfer is less dependent on realized output $y_{p}$. That is, if $r_{1}<r_{2}$ (so $r_{2}$ is more riskaverse), then $r_{2}$ receives a share $\frac{r_{1}}{r_{1}+r_{2}}<\frac{1}{2}$ of realized output, plus a constant. The division of surplus $v$ affects only the constant part of the total transfer.

Since $r_{2}$ 's expected utility is fixed at $v$, it's clear that, for each $v$, both members "agree" on project choice-they want to maximize surplus, given the division. ${ }^{13}$ The optimal project can thus be characterized by maximizing $r_{1}$ 's objective function under $s^{*}\left(y_{p} ; v\right)$. Crucially, this shows that there exists a unique optimal project for the pair, which depends only on their risk tolerances and not on the division $v$.

We can now express the certainty-equivalent for each member of the pair, given $v$ :

$$
\begin{aligned}
C E_{r_{1}}(v) & =-\left(\frac{1}{r_{1}}+\frac{1}{r_{2}}\right) \log \int_{-\infty}^{\infty} e^{-\frac{r_{1} r_{2}}{r_{1}+r_{2}} y_{p}} f\left(y_{p} \mid p^{*}\left(r_{1}, r_{2}\right)\right) d y_{p}-\frac{1}{r_{2}} v \\
C E_{r_{2}}(v) & =\frac{1}{r_{2}} v
\end{aligned}
$$

This makes it clear that $r_{2}$ gains one unit of certainty-equivalent at the expense of exactly one unit of certainty-equivalent for $r_{1}$. Since the certainty-equivalent is just a monotonic transformation of expected utility, this shows the transferability of expected utility. Hence, conditions under which $C E: G 1 \times G 2 \rightarrow \mathbb{R}$ exhibits increasing and decreasing differences in risk types are necessary and sufficient for unique PAM and NAM, respectively. Proposition 1 provides these conditions.

Proposition 1 Recall that $V(p)$ describes the variance cost of a project with mean return $p$. Then $M(p) \equiv V^{\prime}(p)$ describes the marginal variance cost of a project with mean return $p$.

(a) A sufficient condition for PAM to be the unique equilibrium match is $M^{\prime \prime}(p)<0$ for $p>0$ (concavity).

(b) A sufficient condition for NAM to be the unique equilibrium match is $M^{\prime \prime}(p)>0$ for $p>0$ (convexity).

(c) A sufficient condition for any match to be sustainable as an equilibrium is $M^{\prime \prime}(p)=0$ for $p>0$ (linearity).

Note that while the curvature of marginal variance cost is sufficient for assortative matching in a known population of individuals, it is both necessary and sufficient to guarantee assortative matching for any possible population $G 1 \times G 2 \subset \mathbb{R}^{+} \times \mathbb{R}^{+}$(for the matching conditions to be independent of what risk types are in the population).

Note that Proposition 1 tells us that equilibrium matching depends only on the relationship between the mean and the variance of the risky project returns, even though the return distributions may have infinitely many nonzero higher order cumulants (Appendix 9 shows why this is not a direct consequence of CARA preferences).

\footnotetext{
${ }^{13}$ Wilson (1968) showed that, as a consequence of CARA utility, any pair acts as a syndicate and "agrees" on project choice.
} 
The following corollary provides intuition for this result by relating the curvature of the marginal variance function to properties of the risk environment.

Corollary 1 Let $C V(p)=\frac{V(p)^{\frac{1}{2}}}{p}$ represent the coefficient of variation of a project $p$. Then:

(a) $C V^{\prime}(p)<0 \forall p>0$ iff $M^{\prime \prime}(p)<0 \forall p>0(P A M)$

(b) $C V^{\prime}(p)>0 \forall p>0$ iff $M^{\prime \prime}(p)>0 \forall p>0(N A M)$

Recall that the coefficient of variation is unitless (dimensionless), and thus a convenient tool for comparing portfolios.

As the proof of Proposition 1 involves a novel approach to portfolio problems with higher order cumulants, I sketch it here. Details for the proofs of the proposition and the corollary can be found in Appendix 4.

Define the representative risk tolerance of a matched pair $\left(r_{1}, r_{2}\right)$ :

$$
\begin{aligned}
R & \equiv R_{1}+R_{2} \\
& \equiv \frac{1}{r_{1}}+\frac{1}{r_{2}}
\end{aligned}
$$

Then observe that the sum of certainty-equivalents of the pair, $C E\left(r_{1}, r_{2}\right)$, depends only on each individual's risk tolerance through the representative risk tolerance of the pair:

$$
\begin{aligned}
C E\left(r_{1}, r_{2}\right) & =C E\left(R\left(r_{1}, r_{2}\right)\right) \\
& =-R \log \int_{-\infty}^{\infty} e^{-\frac{1}{R} y_{p}} f\left(y_{p} \mid p^{*}(R)\right) d y_{p}
\end{aligned}
$$

Moreover, $C E\left(r_{1}, r_{2}\right)$ exhibits supermodularity (submodularity) in $r_{1}, r_{2}$ precisely when $C E(R)$ exhibits convexity (concavity) in $R$.

The key is to observe that $C E(R)$ is the product of $-R$ and the cumulant-generating function $K_{Y_{p}}(t)$ of the distribution of project returns $Y_{p}$. The cumulant-generating function (cgf) is the log of the moment-generating function (mgf), and the first two cumulants of any distribution are the mean and the variance. Since $Y_{p}=p+V(p)^{\frac{1}{2}} Y$, the sequence of cumulants for each $Y_{p}$ can be expressed as a function of the sequence of cumulants for $Y$.

Using the series expansion of the cgf, where $k_{n}(y)$ denotes the $n^{\text {th }}$ cumulant of $Y$, we see that:

$$
C E(p, R)=p-\sum_{n=2}^{\infty} \frac{(-1)^{n}}{n ! R^{n-1}} V(p)^{\frac{n}{2}} k_{n}(y)
$$

A pair with representative risk tolerance $R$ chooses $p$ to maximize $C E(p, R)$. Thus, the optimal project balances the marginal benefit of increased mean with the marginal cost of higher "generalized variance" (the aggregated higher-order cumulants which form a polynomial in $V(p)$ ), where a given level of "generalized variance" is less costly for more risk-tolerant pairs. Hence, the curvature of the marginal variance cost $M(p)$ is at the heart of the curvature of $C E(R)$ and of assortative matching. 
Corollary 1 sheds light on how the curvature of $M(p)$ shapes the set of portfolios available to a population, providing further intuition for the connection between $M(p)$ and equilibrium matching. A less risk-averse person enjoys the premium a more risk-averse partner is willing to pay her to smooth his consumption, but acting as the informal insurer and bearing her partner's risk forces the pair to choose a safer project with lower expected return. If she instead matches with a less risk-averse partner, she forgoes the premium from providing insurance, but she and her partner are able to undertake a riskier project with higher expected return. Whether a less risk-averse individual prefers to be an informal insurer or an entrepreneur, and thus whether negative or positive assortative matching results, depends on whether partnerships generate the most value through insurance or production. When the ratio of standard deviation to expected return is higher for projects with higher expected return, the less risk-averse will prefer to be informal insurers; when the ratio is lower, the less risk-averse will prefer to be entrepreneurs and choose high risk, high return projects.

Thus, the key trade-off is between sharing a given risk, and choosing what risk to share. Propositions 2 and 3 make this clear by shutting down each channel in turn and characterizing the equilibrium match. I show that, when individuals face a fixed risk and partner choice affects only how that risk is shared, negative assortative matching is the unique equilibrium, and the less riskaverse act as informal insurers. When individuals face a fixed sharing rule and partner choice affects only what risk is faced, positive assortative matching is the unique equilibrium, and the less risk-averse are entrepreneurs.

Proposition 2 Suppose that all agents in $G 1$ draw income iid from a distribution $M$, and all agents in $G 2$ draw income iid from a distribution $W$, so that all possible pairs face the same joint income distribution. Once matched, agents can commit ex ante to a return-contingent sharing rule. Then the unique equilibrium matching is NAM.

See Appendix 5 for the proof.

This coincides with the theoretical finding of Legros and Newman (2007), Schulhofer-Wohl (2006), and Chiappori et al. (2006), and with the empirical finding of Ackerberg and Botticini (2002), who find that heterogenously risk-averse landlords and tenant farmers who chose sharing rules but who couldn't choose which crop portfolios to grow matched negative assortatively in medieval Tuscany.

Proposition 3 Suppose that the slope of the sharing rule $s\left(R_{p}\right)=a+b R_{p}$ is fixed. For example, $a$ wage law requires a 50-50 split of output. However, a matched pair is able to choose a risky project $p$, where $Y_{p}=p+V(p)^{\frac{1}{2}} Y$ as in the benchmark model. Then the unique equilibrium matching is PAM.

See Appendix 6 for the proof.

When the dependence of each agent's consumption on realized return is fixed exogenously, less and more risk-averse agents can no longer capitalize on gains from trade: more risk-averse agents 
are unable to offer the less risk-averse higher premiums to bear their risk. Hence, partnerships are motivated by production (project choice), and positive assortative matching is the unique equilibrium. This aligns with the experimental finding of Attanasio et al. (2012), who find that agents match positive assortatively in risk preference when they can choose the riskiness of the gamble they face, but the sharing rule is fixed at equal division.

Finally, Proposition 4 tells us that positive (negative) assortative matching corresponds to a convex (concave) relationship between the mean joint incomes of matched pairs and the representative risk tolerances of those matched pairs.

Proposition 4 Let $p^{*}\left(r_{i}, \mu\left(r_{i}\right)\right)$ denote the mean return of the project chosen by a matched pair $\left(r_{i}, \mu\left(r_{i}\right)\right)$. Then:

(a) If $M^{\prime \prime}(p)<0$ for $p>0$, then $p^{*}\left(r_{i}, \mu\left(r_{i}\right)\right)$ is convex in representative risk tolerance $R\left(r_{i}, \mu\left(r_{i}\right)\right)$.

(b) If $M^{\prime \prime}(p)>0$ for $p>0$, then $p^{*}\left(r_{i}, \mu\left(r_{i}\right)\right)$ is concave in representative risk tolerance $R\left(r_{i}, \mu\left(r_{i}\right)\right)$.

See Appendix 7 for the proof.

This result suggests a falsifiability test for the theory which requires observing only the actual matching and mean incomes (e.g. average household income). Importantly, the test would not require any data on higher-order moments of income distributions (such as the variance), or any counterfactual matching data (such as how agents would have behaved had they been matched with other partners). Falsifiability is discussed in detail in Section 6.

\section{Policy}

Using the paper's theoretical framework and main results, this section demonstrates concretely how the evaluation of a policy's welfare impact may change substantially depending on whether the endogenous response of informal institutions is accounted for. An important and salient example of such a policy is crop price stabilization, which directly affects the risk environment farmers face. Many of the world's poor depend on agriculture for a livelihood, and a common governmental measure to reduce the risk burden for poor farmers is to stabilize crop prices by imposing price floors and ceilings. Notable examples include the stabilization of maize, sorghum, and rice prices

in Venezuela, the Andean Price Band system between Colombia, Ecuador, and Venezuela, and bananas and grains in Ethiopia (Knudsen and Nash (1990), Minot (2010)).

In this section, I study a hypothetical policy which places price bands on crops (maintaining the mean profitability of each crop but reducing each variance), and show that the policy is a strict Pareto improvement when informal relationships are static, but that in fact the most risk-averse agents are made worse off, potentially significantly, once the re-formation of informal relationships is accounted for. Price stabilization has a clear and direct impact on the spectrum of risky portfolio choices, which makes it an appealing policy to study as an example. This exercise is not meant to 
be a thorough analysis of crop price stabilization, which can take many different forms, but rather is meant to provide a clear illustration of the channels by which using the theory to account for endogeneity of an informal institution can alter policy evaluation.

Suppose a benevolent government contemplates solutions for its many poor farmers, who face an unforgiving risk environment, and who lack access to formal insurance. ${ }^{14}$ Because a slight increase in mean profitability of crop portfolio comes at the cost of extremely high variance, farmers grow crops that are safer but not very profitable-they forgo innovations for less profitable, traditional seeds and methods. To encourage the take up of seeds and methods with higher expected return, the government places price bands of the form $\left[p_{L}, p_{H}\right]$ on each crop's price. If the world price of a crop happens to fall within this band, that is the price the farmer faces. However, if the world price falls below the price floor, the farmer is guaranteed to receive $p_{L}$, and if the world price is above the price ceiling, the farmer faces $p_{H}$. The marginal impact of stabilization is largest for crops with the most volatile prices: the variance of every crop falls, but the variance of the riskiest crops falls by the largest amount. Thus, the policy may lead to a change in the curvature of the marginal variance cost $M(p)$ across different crop portfolios.

To see this more concretely, let $Q_{i}$ represent the yield of crop $i$, and $P_{i}$ the net price of crop $i$. Assume that an individual farmer's production does not affect the world price, so that $P_{i}$ is independent of $Q_{i}$. Then, for a given production level $Q_{i}$ (letting $\pi(i)$ denote the profit of $\operatorname{crop} i$ ):

$$
\begin{aligned}
E(\pi(i)) & =E\left(P(i) Q_{i}\right) \\
& =Q_{i} E(P(i)) \\
V(\pi(i)) & =V\left(P(i) Q_{i}\right) \\
& =Q_{i}^{2} V(P(i))
\end{aligned}
$$

Now, suppose $P_{i} \sim$ uni $[a(i), b(i)]$, where $0 \leq a(i) \leq b(i)$ for each crop $i$. Then:

$$
\begin{aligned}
E(\pi(i)) & =Q_{i} \frac{a(i)+b(i)}{2} \\
V(\pi(i)) & =\frac{Q_{i}^{2}}{12}(b(i)-a(i))^{2}
\end{aligned}
$$

Under what conditions do crops with higher mean have higher variance? Suppose that crops are ordered from lowest to highest expected return: that is, if $i<i^{\prime}$, then $E(\pi(i))<E\left(\pi\left(i^{\prime}\right)\right)$. Then:

$$
\begin{aligned}
& \frac{\partial E(\pi(i))}{\partial i}>0 \Leftrightarrow a^{\prime}(i)+b^{\prime}(i)>0 \\
& \frac{\partial V(\pi(i))}{\partial i}>0 \Leftrightarrow b^{\prime}(i)>a^{\prime}(i)
\end{aligned}
$$

\footnotetext{
${ }^{14}$ I abstract from possible governmental and other frictions in policy implementation, in order to study how accounting for the equilibrium response of informal institutions might substantially alter policy analysis even in a "politically ideal" world.
} 
For example, this is satisfied for $a(i), b(i)$ where $b^{\prime}(i)>a^{\prime}(i) \geq 0$.

By Corollary 1, we know that a change in the curvature of the marginal variance cost across the different crops is equivalent to a change in the monotonicity of the coefficient of variation across crops. For example, if pre-policy, the coefficient of variation is lower for projects with lower expected return, and post-policy, the coefficient of variation is lower for projects with higher expected return, then the unique matching pre-policy is negative-assortative, while post-policy, it is positive-assortative.

So, when is the coefficient of variation lower for projects with lower expected return, and when is it lower for projects with higher expected return? Assuming $b^{\prime}(i)>a^{\prime}(i) \geq 0$ (so that crop $i^{\prime}$ has higher mean and variance than $i$ if $i^{\prime}>i$ ):

$$
\begin{aligned}
C V(i) & =\frac{V(\pi(i))^{\frac{1}{2}}}{E(\pi(i))} \\
& =\frac{(b(i)-a(i))}{\sqrt{3}(a(i)+b(i))} \\
\frac{\partial C V(i)}{\partial i} & >0 \Leftrightarrow a^{\prime}(i) b(i)<a(i) b^{\prime}(i) \\
\frac{\partial C V(i)}{\partial i} & <0 \Leftrightarrow a^{\prime}(i) b(i)>a(i) b^{\prime}(i)
\end{aligned}
$$

Natural choices for $a(i), b(i)$ which satisfy these conditions exist. Suppose that pre-policy, the price of each crop $i$ is distributed:

$$
P_{i}^{\text {pre }} \sim[1, \bar{P}(i)], \bar{P}(i) \geq 1, \bar{P}^{\prime}(i)>0
$$

The government imposes a price floor and a price ceiling, to reduce price volatility, so that post-policy:

$$
P_{i}^{p o s t} \sim\left[\frac{\bar{P}(i)+1}{2}-c, \frac{\bar{P}(i)+1}{2}+c\right], c \in\left[0, \frac{\bar{P}(i)-1}{4}\right)
$$

Note that this policy maintains the expected profitability of each crop $\left(E\left(P_{i}\right)=\frac{\bar{P}(i)+1}{2}\right.$ in both cases), but reduces each variance $\left(c<\frac{\bar{P}(i)-1}{4}\right)$. Further, note that pre-policy, the coefficient of variation is smaller for crops with lower expected return, but post-policy, the coefficient of variation is smaller for crops with higher expected return. ${ }^{15}$

Thus, by Corollary 1, the unique matching pattern pre-policy is negative-assortative, and postpolicy, it is positive-assortative: informal insurance relationships endogenously re-form in response to the policy.

Note that we can express the spectrum of risky crop portfolios in the "language" of the theo-

\footnotetext{
${ }^{15} \mathrm{It}$ is straightforward to check that the conditions hold:

$$
\begin{aligned}
& 0<\bar{P}^{\prime}(i) \Leftrightarrow a_{\text {pre }}^{\prime}(i) b_{\text {pre }}(i)<a_{\text {pre }}(i) b_{\text {pre }}^{\prime}(i) \\
& \frac{\bar{P}^{\prime}(i)}{2}\left(\frac{\bar{P}(i)+1}{2}+c\right)>\frac{\bar{P}^{\prime}(i)}{2}\left(\frac{\bar{P}(i)+1}{2}-c\right) \Leftrightarrow a_{\text {post }}^{\prime}(i) b_{\text {post }}(i)>a_{\text {post }}(i) b_{\text {post }}^{\prime}(i)
\end{aligned}
$$
}


retical framework: $Y_{i}=E\left(Y_{i}\right)+V\left(Y_{i}\right)^{\frac{1}{2}} Y_{0}$, where $Y_{i}$ represents the profit of crop $i$, and $Y_{0}$ is the uniform random variable with $E\left(Y_{0}\right)=0, V\left(Y_{0}\right)=1 .{ }^{16}$ Then, pre-policy, the spectrum of risky projects can be described by:

$$
Y_{i}=Q_{i} \frac{\bar{P}(i)+1}{2}+Q_{i} \frac{(\bar{P}(i)-1)}{\sqrt{12}} Y_{0}
$$

And post-policy, the spectrum of risky projects can be described by:

$$
Y_{i}=Q_{i} \frac{\bar{P}(i)+1}{2}+Q_{i} \frac{c}{\sqrt{3}} Y_{0}
$$

Now that we've seen how crop portfolio choice maps into the theoretical framework, and how crop price stabilization may generate an important change in the set of risky portfolios, let us compare the welfare impacts of the risk-reduction policy in a more general environment, ignoring and then accounting for the endogeneity of informal insurance institutions.

Assume that the returns of different crop portfolios are distributed normally. ${ }^{17}$ In particular, suppose that pre-policy, the profits of a crop with mean $p$ are described by $\pi_{p}^{p r e} \sim N\left(p, p^{N_{1}}\right)$, $N_{1}>2$. Thus, the marginal variance cost function is $M_{\text {pre }}(p)=N_{1} p^{N_{1}-1}$, which is convex in $p$. The government then implements a stabilization policy to differentially reduce the risk of higher mean portfolios. Post-policy, profits are described by $\pi_{p}^{\text {post }} \sim N\left(p, p^{N_{2}}\right)$, where $N_{2} \in(1,2)$, so that $M_{\text {post }}(p)$ is now concave in $p \cdot{ }^{18}$ According to Proposition 1 , the unique equilibrium match is negative assortative pre-policy, and positive assortative post-policy ( $N=2$ is the "tipping point", at which every matching pattern is stable).

Importantly, the risk-reduction policy affects level as well as curvature of risk. Reducing the variance of every portfolio to 0 would clearly make everyone happier: the positive impact of the change in levels outweighs any impact the change in curvature might have. However, policy is generally only able to achieve small reductions in risk levels before returns are affected. Moreover, we know from Proposition 1 that the composition of partnerships is driven by curvature, not levels. Thus, setting $N_{1}=2+\varepsilon$ and $N_{2}=2-\varepsilon, \varepsilon$ small, maximizes the change in curvature resulting from the policy relative to the change in levels, and enables us to isolate the impact stemming from the endogenous response of the informal insurance network. For this example, let $\varepsilon=0.1$.

\footnotetext{
${ }^{16}$ That is, $Y_{0} \sim$ uni $\left[-\frac{\sqrt{12}}{2}, \frac{\sqrt{12}}{2}\right]$.

${ }^{17}$ This simplifies the form of the joint certainty-equivalent of a matched pair, since the normal distribution is the only absolutely continuous distribution such that the only nonzero cumulants are the mean and the variance.

${ }^{18}$ Note that modeling $V(p)$ as a power function has one small drawback. It's a natural choice, since we want to analyze a policy that reduces the variance of every project, and particularly reduces the variance of the riskiest projects, which is captured by "decreasing $N^{\prime}$. However, when $N$ falls, $p^{N}$ for $p \in(0,1)$ actually increases. Hence, assume that $G 1$ and $G 2$ are such that no possible pair ever wishes to undertake a project $p \in(0,1)$ pre-policy:

$$
\frac{1}{\max \left(r_{1}\right)}+\frac{1}{\max \left(r_{2}\right)} \geq \frac{N_{1}}{2}
$$

This is for convenience, and does not substantively influence the intuition or the policy analysis, since the matching results are free of any assumption on the distribution of risk types. If the above condition did not hold, the policy would be a bad idea without considering informal institutions, since individuals choosing $p<1$ would be worse off.
} 
Suppose $G 1=\{0.6,0.7,0.8,0.9\}$, and $G 2=\{1,1.1,2,2.1\}$. For example, $G 1$ may be the less risk-averse landowners in a region, and $G 2$, the more risk-averse tenant farmers. (However, the matching results and intuition about the policy do not depend on which risk types are chosen). Then the effect of the policy without accounting for the response of informal institutions is described by the lemma below.

Lemma 2 A policy which reduces the variance of every available project is a strict Pareto improvement if the composition of partnerships does not change.

The proof is intuitive: each matched partnership can stay on the same project post-policy, or can choose a different project. If a partnership retains its original project, it is strictly better off, since the project has the same mean as it did before the policy, but a lower variance. If a partnership switches to a different project, then by revealed preference, they must be even better off facing the new project than facing the old project with decreased variance. But this means that each partnership is strictly better off.

Now, suppose individuals re-form partnerships in response to the policy. How does this affect the evaluation of the policy impacts?

First, consider the effect on individual payoffs. Note that, while Proposition 1 provides conditions under which the equilibrium matching and joint payoffs are unique, individual payoffs are never unique. Instead, the stability conditions (that is, the requirement that no two unmatched agents prefer to defy the match and partner with each other instead) determine the sets of individual payoffs which support the equilibrium matching. Thus, once the payoff of one agent is fixed, the equilibrium payoffs of the $(2 N-1)$ other agents in the population are determined.

To be perfectly clear about individual payoffs in this setting, let $C E_{i j}^{\text {pre }}$ and $C E_{i j}^{\text {post }}$ denote the certainty-equivalent generated by a partnership between the $i^{\text {th }}$ agent in $G 1$ and the $j^{\text {th }}$ agent in $G 2$ in the pre- and post-policy cases, respectively (note that agents are ordered from least to most riskaverse. The certainty-equivalent generated by a matched partnership $(i, j)$ differs pre-policy and post-policy because the policy alters the spectrum of portfolio choices by altering the mean-variance tradeoff across risky projects). Then the pre-policy individual payoffs of the eight individuals in this example (who are matched negative-assortatively, by Proposition 1) can be described by:

$$
\begin{aligned}
& \left\{\begin{array}{l}
u_{G 1,1}=C E_{14}^{p r e}-v_{4} \\
u_{G 1,2}=C E_{23}^{\text {pre }}-\left(C E_{13}^{\text {pre }}-C E_{14}^{\text {pre }}\right)-v_{4} \\
u_{G 1,3}=C E_{32}^{\text {pre }}-\left(C E_{22}^{\text {pre }}-C E_{23}^{\text {pre }}\right)-\left(C E_{13}^{\text {pre }}-C E_{14}^{\text {pre }}\right)-v_{4} \\
u_{G 1,4}=C E_{41}^{\text {pre }}-\left(C E_{31}^{\text {pre }}-C E_{32}^{\text {pre }}\right)-\left(C E_{22}^{\text {pre }}-C E_{23}^{\text {pre }}\right)-\left(C E_{13}^{\text {pre }}-C E_{14}^{\text {pre }}\right)-v_{4}
\end{array}\right. \\
& \left\{\begin{array}{l}
u_{G 2,1}=\left(C E_{31}^{\text {pre }}-C E_{32}^{\text {pre }}\right)+\left(C E_{22}^{\text {pre }}-C E_{23}^{\text {pre }}\right)+\left(C E_{13}^{\text {pre }}-C E_{14}^{\text {pre }}\right)+v_{4} \\
u_{G 2,2}=\left(C E_{22}^{\text {pre }}-C E_{23}^{\text {pre }}\right)+\left(C E_{13}^{\text {rre }}-C E_{14}^{\text {pre }}\right)+v_{4} \\
u_{G 2,3}=\left(C E_{13}^{\text {pre }}-C E_{14}^{\text {pre }}\right)+v_{4} \\
u_{G 2,4}=v_{4}
\end{array}\right.
\end{aligned}
$$


Recall that $C E_{i j}>C E_{i j^{\prime}}$ if $j$ is less risk-averse than $j^{\prime}\left(j<j^{\prime}\right)$. The structure of the individual payoffs is nicely intuitive: for example, consider the agents in $G 2$. The payoff of the most riskaverse agent $(G 2,4)$ is fixed at $v_{4}$. Then the next most risk-averse agent, $(G 2,3)$, receives the most risk-averse agent's payoff, plus an extra wedge to make $(G 1,1)$, the least risk-averse person in $G 1$ (who is partnered with the most risk-averse person in $G 2$, in the equilibrium negative-assortative match), indifferent between partnering with $(G 2,4)$ and $(G 2,3)$ : the most $(G 1,1)$ could gain from switching to $(G 2,3)$ is $C E_{13}-C E_{14}$. Similarly, $(G 2,2)$ receives $(G 2,3)$ 's payoff, with an extra wedge to prevent deviation, and so on.

Post-policy, the equilibrium match is positive-assortative, and individual payoffs are described by:

$$
\begin{aligned}
& \left\{\begin{array}{l}
u_{G 1,1}=C E_{11}^{\text {post }}-\left(C E_{21}^{\text {post }}-C E_{22}^{\text {post }}\right)-\left(C E_{32}^{\text {post }}-C E_{33}^{\text {post }}\right)-\left(C E_{43}^{\text {post }}-C E_{44}^{\text {post }}\right)-v_{4} \\
u_{G 1,2}=C E_{22}^{\text {post }}-\left(C E_{32}^{\text {post }}-C E_{33}^{\text {post }}\right)-\left(C E_{43}^{\text {post }}-C E_{44}^{\text {post }}\right)-v_{4} \\
u_{G 1,3}=C E_{33}^{\text {post }}-\left(C E_{43}^{\text {post }}-C E_{44}^{\text {post }}\right)-v_{4} \\
u_{G 1,4}=C E_{44}^{\text {post }}-v_{4}
\end{array}\right. \\
& \left\{\begin{array}{l}
u_{G 2,1}=\left(C E_{21}^{\text {post }}-C E_{22}^{\text {post }}\right)+\left(C E_{32}^{\text {post }}-C E_{33}^{\text {post }}\right)+\left(C E_{43}^{\text {post }}-C E_{44}^{\text {post }}\right)+v_{4} \\
u_{G 2,2}=\left(C E_{32}^{\text {post }}-C E_{33}^{\text {post }}\right)+\left(C E_{43}^{\text {post }}-C E_{44}^{\text {post }}\right)+v_{4} \\
u_{G 2,3}=\left(C E_{43}^{\text {post }}-C E_{44}^{\text {post }}\right)+v_{4} \\
u_{G 2,4}=v_{4}
\end{array}\right.
\end{aligned}
$$

When equilibrium individual payoffs are nonunique, the convention is to focus on specific cases. Below, I consider two standard cases: the "best case scenario for the most risk-averse agent" (Case 1), and the "worst case scenario for the most risk-averse agent" (Case 2). All other cases are intermediate. The "best case scenario for the most risk-averse agent" is the vector of payoffs in which the most-risk averse agent (agent 4 in $G 2$ ) receives a payoff that is larger than the payoff she receives in any of the other possible vectors of equilibrium individual payoffs. More specifically, in Case 1, the "best case scenario for the most risk-averse agent":

$$
\begin{aligned}
v_{4}^{\text {pre }} & =C E_{14}^{\text {pre }} \\
v_{4}^{\text {post }} & =C E_{44}^{\text {post }}
\end{aligned}
$$

Figures 2a and 2b plot the payoffs of each individual in $G 1$ and $G 2$ against each individual's coefficient of absolute risk aversion. The red line with circular markers depicts pre-policy payoffs, while the blue line with square markers depicts post-policy payoffs. Recall that the less risk-averse agents are in $G 1$, and the more risk-averse agents are in $G 2$. 

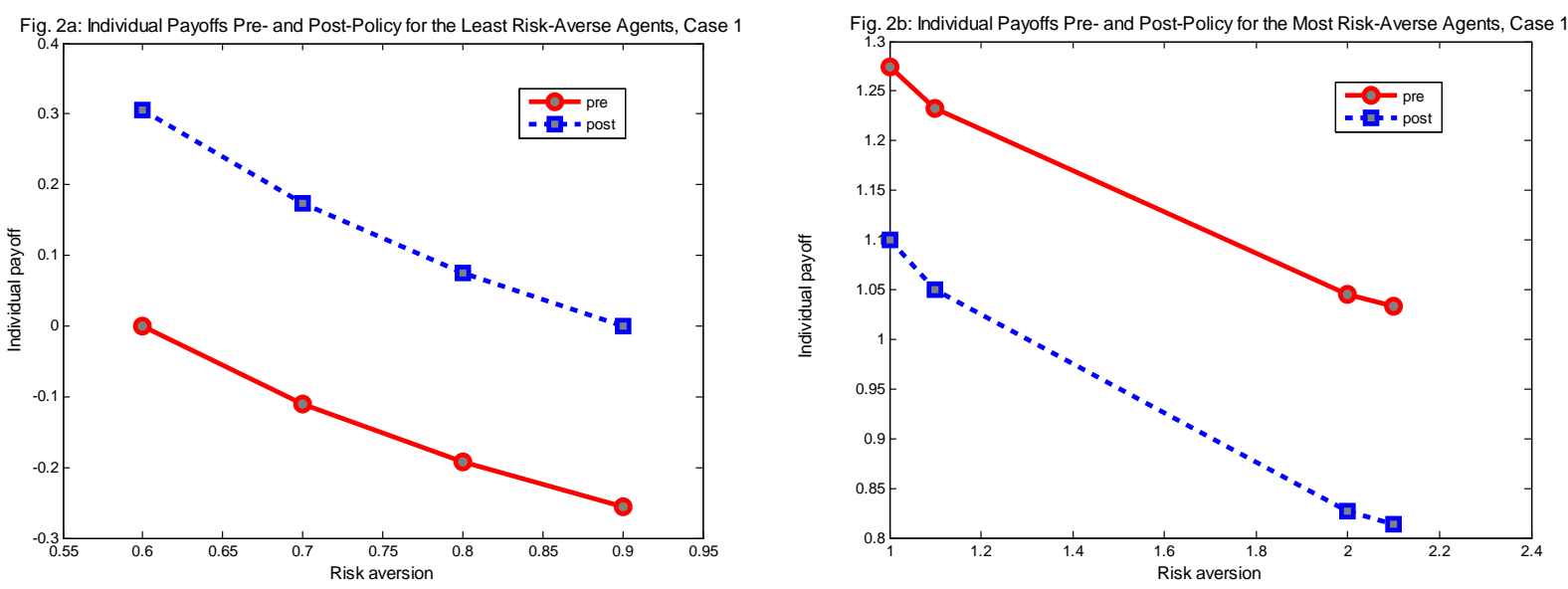

What happens in Case 2, the "worst case scenario for the most risk-averse agent"? The "worst case scenario for the most risk-averse agent" is the vector of payoffs in which the most-risk averse agent (agent 4 in $G 2$ ) receives a payoff that is smaller than the payoff she receives in any of the other possible vectors of equilibrium individual payoffs. More specifically, in the "worst case scenario for the most risk-averse agent":

$$
\begin{aligned}
v_{4}^{\text {pre }} & =-\left(C E_{31}^{\text {pre }}-C E_{32}^{\text {pre }}\right)-\left(C E_{22}^{\text {pre }}-C E_{23}^{\text {pre }}\right)-\left(C E_{13}^{\text {pre }}-C E_{14}^{\text {pre }}\right) \\
v_{4}^{\text {post }} & =-\left(C E_{21}^{\text {post }}-C E_{22}^{\text {post }}\right)-\left(C E_{32}^{\text {post }}-C E_{33}^{\text {post }}\right)-\left(C E_{43}^{\text {post }}-C E_{44}^{\text {post }}\right)
\end{aligned}
$$

Figures 3a and 3b plot the payoffs of each individual in $G 1$ and $G 2$ against each individual's coefficient of absolute risk aversion. The red line with circular markers depicts pre-policy payoffs, while the blue line with square markers depicts post-policy payoffs.
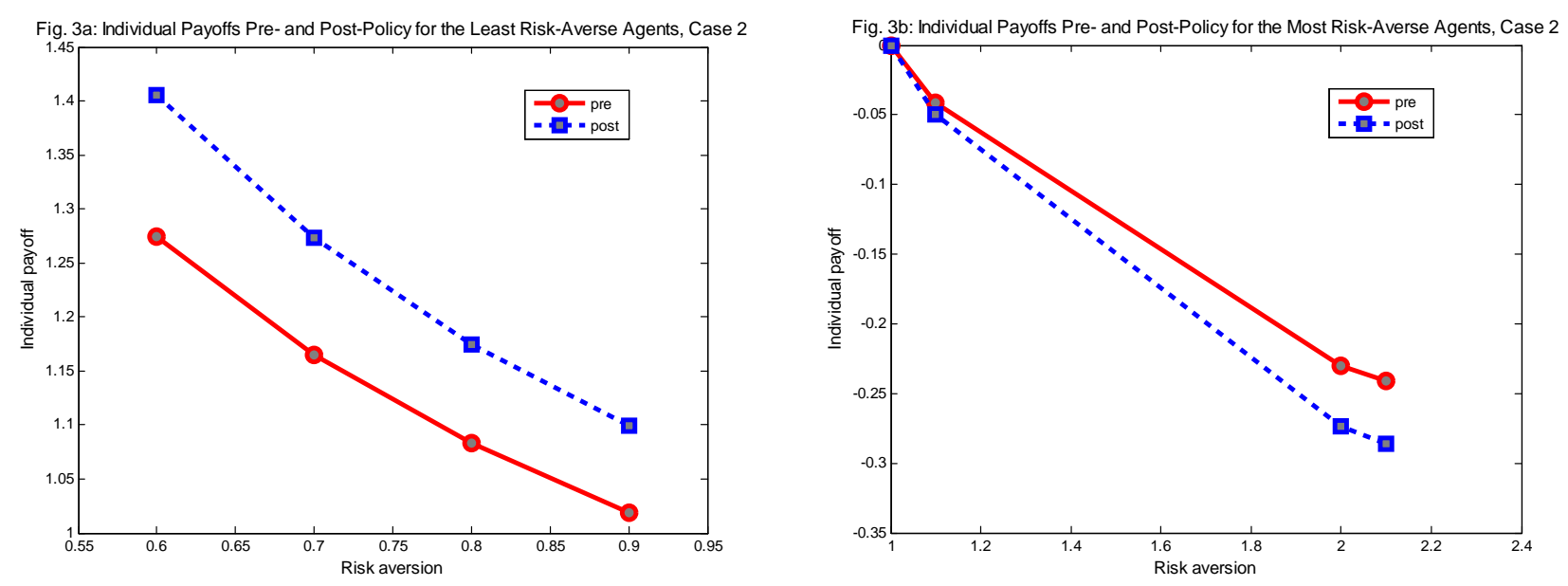
Clearly, this risk-reduction policy is not a Pareto improvement. ${ }^{19}$ We see that the more riskaverse agents are worse off after implementation of the policy, purely as a result of the endogenous network response: the policy causes the least risk-averse agents to abandon their roles as informal insurers of the most risk-averse agents, in favor of entrepreneurial partnerships with fellow less risk-averse agents. The poorest, most risk-averse agents are harmed via two channels: first, they've lost their informal insurers, and this weakens their capacity to smooth consumption, which reduces their welfare. To make matters worse, because the most risk-averse agents, who are now paired with each other, have very little capacity to smooth a given risk (as neither is willing to bear the volatility to smooth her partner's consumption), they must instead manage risk by choosing projects with low variance, which traps them into growing crop portfolios with low mean returns.

On the other hand, the least risk-averse agents, who are now paired with each other, no longer play the role of informal insurer, and this enables them to take advantage of the decreased aggregate risk and undertake the higher mean, entrepreneurial projects (e.g. adopt a new technology). They are better off post-policy. Thus, we see that the emergence of enterpreneurship corresponds to gains for the less risk-averse and losses for the more risk-averse.

How do the risky projects (in this case, crop portfolios) chosen in the economy differ pre- and post-policy? The change is depicted in Figure 4:

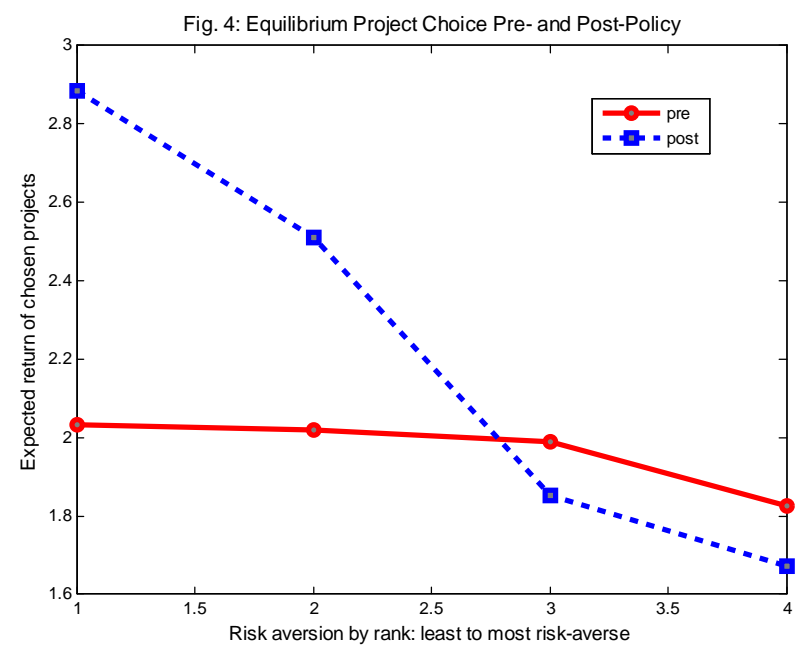

There is much more variation in the projects which are undertaken post-policy: the least riskaverse pairs choose riskier, more entrepreneurial projects post-policy, while the most risk-averse pairs choose safer projects.

These insights provide an interesting complement to existing literature. Attanasio and Rios-Rull (2000) model the introduction of formal insurance as a policy which reduces the aggregate riskiness of the environment. They also find that such a policy may hurt the welfare of the most risk-averse agents. However, their model, which builds off Ligon, Thomas, Worrall (2001), considers a fixed

\footnotetext{
${ }^{19}$ See Appendix 11 for a discussion of the general conditions under which a policy that triggers a change in partnership composition improves the welfare of the less risk-averse at the expense of the more risk-averse.
} 
group of risk-sharing members whose informal insurance arrangement is constrained by limited commitment. Two agents sustain informal risk-sharing by threatening credibly to cut off all future ties if someone reneges, that is, does not honor the risk-sharing agreement (e.g. a member keeps her own income realization instead of transferring some of it to an unlucky partner). Thus, anything that lowers the cost of autarky (the state of being alone and unable to share risk with somebody else) will decrease the level of informal insurance that can be sustained, because the punishment has become less costly. Since the introduction of formal insurance reduces aggregate risk, such a policy reduces the cost of autarky, and as a consequence informal insurance is weakened.

However, if commitment were perfect in Attanasio and Rios-Rull (2000), the introduction of formal insurance would strictly improve welfare, because lowering the cost of autarky matters only through the punishment of cutting off future ties, which would no longer be relevant. One contribution of this example, then, is to show that, even when commitment is perfect, introducing formal insurance might still reduce the welfare of the most risk-averse agents, because the composition of the informal risk-sharing network changes in response. Reducing the riskiness of the environment does increase the value of autarky, but it also increases the value of being in a relationship, and increases it heterogeneously across partnerships of different risk compositions.

This example also contrasts with Chiappori et al. (2011), who estimate that the least riskaverse individuals are the ones left worse off after the introduction of formal insurance, since they have been displaced as informal insurers. However, this illuminates the need for a model of the equilibrium network of relationships-I show that the least risk-averse agents do leave their roles as informal insurers, but only because they prefer to undertake entrepreneurial pursuits instead. It would be interesting to see how their estimation of the welfare impacts changes when they account for this endogenous response.

\section{Endogenous Group Size}

The focus thus far has been on conditions under which assortative matching emerges as the unique equilibrium when groups are constrained to be of size two. However, we know that a matched pair behaves like a single agent with representative risk tolerance $R$. In fact, the proof of Lemma 1 shows that this is a property of a matched group of any size (echoing Wilson (1968)). This suggests defining the representative risk tolerance of a group of $N$ matched people:

$$
R=\sum_{i=1}^{N} \frac{1}{r_{i}}
$$

In order to think about equilibrium matching when group size itself is endogenous, we need to make a few adjustments to the benchmark model. Instead of matching across two distinct groups, individuals all belong to one group, $G$, and match within this group (this simply allows for group size to be an odd number). As before, production requires at least two collaborators (discussed in Appendix 1), and a matched group of agents jointly chooses a risky project $p \geq 0$ from a spectrum 
of risky projects, where a project $p$ 's returns are described by $Y_{p}=p+V(p)^{\frac{1}{2}} Y, Y$ a random variable with well-defined cdf and $E(Y)=0, V(Y)=1 .{ }^{20}$ Individuals in an $N$-person group commit to a feasible return-contingent sharing rule $s_{2}\left(y_{p}\right), s_{3}\left(y_{p}\right), \ldots, s_{N}\left(y_{p}\right)$, which describes the share of realized output each member receives, for each possible level of output.

Define an equilibrium matching to be one that is stable to all coalitional deviations. That is, a group is stable if no strict subset of the group is able to break away and choose a project and sharing rule such that every member of the deviating coalition is weakly better off, and at least one member is strictly better off.

Proposition 5 relates familiar conditions on the risk environment to the size and composition of matched groups in equilibrium in this more general model.

Proposition 5 Let $M(p) \equiv V^{\prime}(p)$ describe the marginal variance cost of each project $p$. Then:

(a) If $M^{\prime \prime}(p)<0$, the unique equilibrium is maximal-connectedness: the whole group, $G$, is matched.

(b) If $M^{\prime \prime}(p)>0$, the unique equilibrium is minimal-connectedness. Individuals match in negative assortative pairs: the $i^{\text {th }}$ least risk-averse person in $G$ is matched with the $i^{\text {th }}$ most risk-averse. (If $|G|$ is odd, then the most risk-averse individual in the population remains unmatched.)

See Appendix 8 for the details of the proof.

Proposition 5 shows that when the marginal variance cost is concave in expected return, the unique equilibrium matching coincides with the matching when group size is restricted to two. That is, pairwise matching is actually the optimal matching structure in this case, and the network is minimally-connected. On the other hand, when the marginal variance cost is convex in expected return, all individuals match in one big group, and the network is maximally-connected. Thus, the curvature of the marginal variance cost is tied both to extremal match compositions, as well as to extremal structures of the network of matches.

This result reveals an interesting relationship between the strength of formal insurance institutions and the structure of firms in developing economies. Because poor entrepreneurs lack access to formal insurance, their need to share risk informally influences the nature of the firms they build. When achieving a higher expected return comes at an increasingly steep escalation in risk cost, in the sense that the coefficient of variation (ratio of the standard deviation of returns to the mean) is larger for projects with higher expected return, a less risk-averse individual provides more value by informally insuring a more risk-averse individual. However, this informal insurance provision, which causes the less risk-averse person to bear most of the risk, comes at the cost of investing in higher mean, higher variance projects. Thus, the economy is characterized by a minimally-connected net-

\footnotetext{
${ }^{20}$ The key assumption is that the spectrum of projects available to a group does not depend on its size. Of course, this is more realistic in some settings than others. This can be interpreted several ways. For example, in the context of agriculture, the robustness of different seeds to rainfall and planting time differentiates the riskiness of different crops, and does not depend on the number of people in a group. Alternatively, there may be constraints to diveresification, which is particularly plausible in a developing environment. Thus, adding more people to a group may not affect the spectrum of portfolios available to the group.
} 
work of many small, heterogeneously-composed firms, each choosing safe, non-innovative projects with low mean and low variance of return.

On the other hand, when the coefficient of variation is smaller for projects with higher expected return, the less risk-averse individuals can bear some risk and still be able to choose entrepreneurial projects, since the increase in risk is outweighed by the increase in expected return. In particular, accepting a less risk-averse partner enables the take up of a project with much higher mean return, and this benefit outweighs the cost of having to share good outcomes. Moreover, the less risk-averse are also happy to insure the more risk-averse, since projects with higher mean return no longer come at such a steep escalation in risk cost. Thus, the economy is characterized by a maximally-connected network: one large firm choosing a risky, entrepreneurial project.

This result contrasts interestingly with Genicot and Ray (2003), who find that, under limited commitment, whole-group matching can never be sustained as a coalitionally-stable equilibrium in a model where individuals are homogeneously risk-averse and draw their income realization independently from the same exogenously-specified income distribution. In their framework, because individuals cannot choose what risk to face, the marginal benefit of an additional member is inevitably eventually negative: an individual's expected income is independent of group size, but she becomes increasingly likely to bear a partner's downside risk as the group grows, and limited commitment implies that individuals cannot be compelled to bail partners out after some point. Thus, a subgroup will always profitably deviate if the whole group tries to match, when the number of people in the population is large.

\section{Falsifiability and Empirical Support for the Theory}

While the previous sections are devoted to exploring interesting implications of the theory, this section provides a variety of empirical approaches for testing the theory.

One such approach is to check directly the conditions of Proposition 1 or Corollary 1. That is, the interested researcher could elicit the risk attitudes of individuals in a population (e.g. using Binswanger (1980) gambles, or a host of other techniques in the literature), as well as record network connections between individuals. The remaining component is the mean and variance of return for each of the risky projects individuals are able to undertake. For example, Appendix 13 contains tables showing the mean and variance of yield of a variety of crops available to farmers in different regions-this data has been collected extensively for agriculture. Alternatively, the coefficient of variation of each project could be calculated. This approach also suits a lab experiment, since the researcher is able to design the set of projects offered to different pools of subjects (and thus determine the coeffcients of variation at the outset). Hence, a variety of different methods enable the researcher to construct $V(p)$ (the variance of return associated with a mean return $p$ ), or $C V(p)$ (the coefficient of variation associated with mean return $p$ ), and verify or falsify the theoretical prediction regarding the relationship of the curvature of the marginal variance cost, the monotonicity of the coefficient of variation in expected return, and equilibrium matching patterns. 
However, the researcher may not always be able to reliably construct $V(p)$ or $C V(p)$. Proposition 4 suggests another approach. Instead of constructing $V(p)$, it is only necessary to collect the mean incomes of matched groups, in addition to the data on which individuals are matched and their risk attitudes. Then, the theory predicts that positive assortative matching corresponds with convexity of mean incomes in the representative risk tolerances of matched groups, while negative assortative matching corresponds with concavity. Importantly, this approach does not require knowing anything about the set of risky production opportunities available in the economy, does not require knowing any counterfactuals, such as what projects individuals would have chosen had they matched with different partners, and does not require knowing any moments of the distributions of returns beyond the mean. (Note that another interesting application of Proposition 4 is that it enables policymakers to identify when an economy is near a "tipping point" of the kind discussed in the policy section-when mean incomes are close to being linear in risk tolerances, we can infer that even a small change in the risk environment could "tip" the matching from one extreme to the other, and could lead to the sorts of adverse welfare consequences illustrated by the crop price stabilization example.)

To fix ideas, consider the following example. Suppose $G 1=\left\{r_{1}^{A}, r_{2}^{A}, r_{3}^{A}\right\}$ and $G 2=\left\{r_{1}^{B}, r_{2}^{B}, r_{3}^{B}\right\}$, where $r_{1}^{A, B}<r_{2}^{A, B}<r_{3}^{A, B}$.

Then, suppose the underlying (unobservable) marginal variance cost function is concave: $M^{\prime \prime}(p)<$ 0. Suppose we observe agents matching positive assortatively in risk attitude, as Proposition 1 predicts. Suppose we also observe the mean incomes of each pair, $p\left(r_{1}^{A}, r_{1}^{B}\right), p\left(r_{2}^{A}, r_{2}^{B}\right)$, and $p\left(r_{3}^{A}, r_{3}^{B}\right)$, as well as the risk types of each agent. Note that we aren't able to force the agents to match in different ways, so we cannot observe what they would have chosen with different partners. Furthermore, we do not observe higher-order moments of the income distributions, including the variance.

Hence, we follow the approach suggested by Proposition 4, and use the observed matchings $\left(r_{1}^{A}, r_{1}^{B}\right),\left(r_{2}^{A}, r_{2}^{B}\right)$, and $\left(r_{3}^{A}, r_{3}^{B}\right)$ to calculate the representative risk tolerance $R_{i}=\frac{1}{r_{i}^{A}}+\frac{1}{r_{i}^{B}}$ of each matched pair. Then, to check for concavity or convexity of mean incomes in representative risk tolerances, regress $p_{i} \equiv p\left(r_{i}^{A}, r_{i}^{B}\right)$ on a constant, as well as $R_{i}$ and $R_{i}^{2}$ (to use a crude second-order polynomial approximation):

$$
p_{i}=\beta_{1}+\beta_{2} R_{i}+\beta_{3} R_{i}^{2}+\varepsilon_{i}
$$

If $\hat{\beta}_{3}>0$, this suggests that $p_{i}$ is convex in $R_{i}$, and this is evidence supportive of the theory, since we have established the following: (a) individuals are matched positive assortatively in risk preferences, and (b) the mean incomes of the matched pairs are convex in the representative risk tolerances of those pairs. On the other hand, $\hat{\beta}_{3}<0$ would be evidence against the theory, since individuals are matched positive assortatively, but the mean incomes of the matched pairs are concave in the representative risk tolerances. (Of course, more sophisticated techniques may be used to test for concavity or convexity.)

I apply a combination of these approaches to the dataset from Attanasio et al. (2012) to seek preliminary support or falsification of the theory in the existing literature. Attanasio et al. (2012) 
run a unique experiment with 70 Colombian communities, where they invite individuals to match in risk-sharing groups in a lab setting. Individuals are able to choose what gamble they face from a set of gambles offered by the experimenters, where higher mean gambles come at the cost of higher variance. However, the sharing rule for each risk-sharing group is fixed at equal division. Thus, a group is able to choose what risk to face, but cannot control how to share a given risk. Risk attitudes are elicited by privately asking each subject to select a gamble before the risk-sharing round is played. In addition, data on pre-existing kinship and friendship networks are gathered. Kinship and friendship ties matter for two important reasons: first, individuals are likely to know the risk attitudes of family and friends, and unlikely to know the risk attitudes of strangers. Second, individuals are likely to trust and therefore commit to family and friends over strangers. Indeed, Attanasio et al. find experimentally that family and friends strongly prefer to match with each other rather than with strangers, and that they match positive assortatively in risk attitude.

To provide some theoretical backbone for this finding, Attanasio et al. study a simplified model of their experimental setting. They assume that individuals have CARA utility and either low or high risk aversion (type is binary). Group size is restricted to pairs. Individuals choose which risky project to undertake from a continuous spectrum of projects, but the sharing rule is fixed at equal division. They show that under the assumptions of this model, individuals match positive assortatively, that is, high types match with other high types while low types match with other low types.

Because Attanasio et al. fix the sharing rule at equal division, the data cannot be used for a full test of the model and theoretical predictions of this paper, which studies risk-sharing groups when individuals choose how to share a given risk as well as what risk to face. However, an interesting partial test is still possible: Propositions 2 and 3 are exactly focused on the "corners" of the model, and describe equilibrium matching when the project choice and sharing rule choice channels are each shut down in turn. Additionally, we can test Proposition 4, which describes the relationship between the curvature of mean incomes in the representative risk tolerances of matched groups, and the curvature of marginal variance cost.

Like the model in Attanasio et al., Proposition 3 predicts unique positive assortative matching when the sharing rule channel is shut down. However, Proposition 3 is proved under more general conditions, including arbitrarily many risk types rather than binary type, and this generality is useful for interpreting the experimental results. In particular, Attanasio et al. assume that the sharing rule is exactly equal division, that is, $s\left(Y_{p}\right)=\frac{1}{2} Y_{p}$, and provide a variety of reasons why they don't think side transfers were a concern in practice. Proposition 3 shows that, even with side transfers $c$, as long as the sharing rule is of the form $s\left(Y_{p}\right)=\frac{1}{2} Y_{p}+c$, the unique equilibrium matching should still be positive assortative, as it is in the experimental findings.

While it is encouraging that the matching pattern predicted by Proposition 3 bears out in the experiment, the predictions of Proposition 4 provide a more rigorous test of the theory. For this, we need to characterize $V(p)$ or $C V(p)$, based on the set of gambles designed by the experimenters. The discrete nature of the gambles suggests drawing upon Corollary 1 and checking for one of 
the following relationships in the data: either the coefficient of variation is increasing in expected return and mean incomes are concave in risk tolerance, or the coefficient of variation is decreasing in expected return and mean incomes are convex in risk tolerance.

The set of gambles in Attanasio et al. is described in the table below (payoffs are in Colombian pesos) ${ }^{21}$. Each gamble had an equally-likely bad and good payoff, where riskier gambles had worse bad payoffs but better good payoffs.

\begin{tabular}{|l|l|l|l|}
\hline Gamble & Mean & Standard Deviation & Coeff. of Variation \\
\hline 1 & 3000 & 0 & 0 \\
\hline 2 & 4200 & 1500 & 0.36 \\
\hline 3 & 4800 & 2400 & 0.5 \\
\hline 4 & 5400 & 3600 & 0.67 \\
\hline 5 & 6000 & 5000 & 0.83 \\
\hline
\end{tabular}

It's clear that the coefficient of variation is larger for gambles with higher expected return. Hence, finding that mean incomes are concave in risk tolerance would be evidence for the theory, while finding that mean incomes are convex in risk tolerance would be evidence against the theory.

I regress the mean returns of the chosen gambles on risk tolerance and squared risk tolerance. A positive coefficient $\beta_{3}$ indicates convexity, while a negative $\beta_{3}$ indicates concavity:

$$
p_{i}=\beta_{1}+\beta_{2}\left(\frac{1}{r_{i}}\right)+\beta_{3}\left(\frac{1}{r_{i}}\right)^{2}+\varepsilon_{i}
$$

The OLS estimates are reported in Table 1:

\begin{tabular}{cc} 
Table 1: Testing for Concavity of Mean Project Returns in Risk Tolerance \\
\hline & OLS Estimates \\
\hline Regressor & $-0.001^{* * *}$ \\
& $(-60.73)$ \\
& \\
Sq. risk tolerance & $2.39^{* * *}$ \\
& $(2.75)$ \\
Risk tolerance & $5169.18^{* * *}$ \\
& $(180.91)$ \\
\hline
\end{tabular}

Notes: This table reports the OLS estimates from a regression of mean project returns on squared risk tolerance, risk tolerance, and a constant. Data from Attanasio et. al. (2012).

*significant at $10 \%$; ** significant at $5 \%$; *** significant at $1 \%$

\footnotetext{
${ }^{21}$ The standard deviations reported in the table in Attanasio et al. (2012) are the experimental, not analytical, standard deviations. The discrepancy results from altruism on the part of the experimenters, who were sometimes found to give the subject the high payoff even when the subject lost the gamble. Here, I consider the analytical variance, since Attanasio et al. state that subjects were unaware of the bias in probabilities. Hence, subjects should have chosen gambles based on the analytical variance.
} 
In line with the theoretical prediction, we see that mean project returns are concave in risk tolerance. Moreover, individuals with a higher risk tolerance (less risk-averse individuals) choose riskier (higher mean, higher variance) gambles. Hence, the experimental results lend preliminary support to the theory.

\section{Conclusion}

This paper enriches our understanding of informal insurance by developing and studying a theory of endogenous relationship formation between heterogeneously risk-averse people who lack access to formal insurance and credit markets and choose both what risk to face as well as how to share a given risk. The strength of informal insurance is thus how well-insured a population of riskaverse individuals is when they must rely only on interactions with fellow risk-averse individuals to manage risk, rather than how well-insured individuals are when the formal contracting environment is weak. Importantly, this approach reveals that what we can learn from endogenizing informal insurance isn't limited to insurance: the need to manage risk informally in an economy influences the strength of entrepreneurship, the correspondence of entrepreneurship with income inequality, and the optimal structure of informal firms.

Existing literature has focused largely on analyzing the insurance agreement reached by a fixed group of individuals, isolated outside of the equilibrium network, or by individuals who match with a fixed probability, at some cost of understanding what groups would actually exist and coexist in the first place. By contrast, this paper studies how the network shape that emerges in equilibrium is determined by the possibilities of other shapes the network could have assumed, and identifies important connections between the equilibrium network and the risk environment. In particular, while income distributions may have infinitely many nonzero higher-order cumulants, equilibrium matching is found to be determined by only the first two: a mean-variance trade-off across portfolio choices, which can be captured by the coefficient of variation.

This paper highlights the especial importance of accounting for, rather than abstracting away from, heterogeneity in developing economies. The absence of formal institutions causes individuals to address these unmet needs with interpersonal relationships. Thus, we see that a natural constraint on informal insurance, apart from any aspect of the formal contracting environment, is

simply that all individuals are risk-averse. However, some individuals are more risk-averse than others, and this enables informal insurance and determines its strength. Yet, just like the formal economy, the informal economy too is endogenous: individuals switch between and assume different informal roles in the economy as circumstances change.

Accounting for this endogeneity may substantially alter our design and evaluation of a variety of policies. While crop price stabilization was explicitly discussed in the paper, the framework is also useful for thinking about land reform. For example, Banerjee (2000) points out that the effects of land redistribution cannot be estimated without first understanding the reasons behind the distribution of landholdings in the status quo. If the allocation served a risk-sharing purpose (for 
example, large landowners provided informal insurance to the landless), then land redistribution could actually decrease welfare.

The model also provides an interesting lens through which to view regulation of wages. We know that employment contracts (such as the sharecrop contract) often balance multiple needs, such as insurance and incentive provision. The results of this paper show that legislation which places restrictions on the set of permissible rental contracts diminishes the power of individuals to share a given risk, which may cause the less risk-averse to cease acting as informal insurers.

Finally, while informal insurance served as the matching motivation in this paper, the ideas of the model are more general and can be applied to other important questions. For example, understanding the equilibrium matching between individuals with different risk attitudes and the risky health behaviors they choose may help us design more effective approaches to encouraging vaccination, boosting health investments and sanitation practices, and preventing and treating HIV.

Many challenges remain. This model can be enriched in a variety of ways-by generalizing preferences, by introducing limited commitment, by adding dynamic re-matching and dynamic investment. I leave these tasks for future research. Developing economies are distinguished by uniquely complex environments and uniquely lofty stakes. That is why they are formidable, and that is why they are important. 


\section{References}

1. "2012 National Trade Estimate Report: Foreign Trade Barriers." 2012. U.S. Wheat Associates.

2. Abebe, T., K.F. Wiersum, and F. Bongers. 2010. "Spatial and Temporal Variation in Crop Diversity in Agroforestry Homegardens of Southern Ethiopia." Agroforestry Systems 78: 309322.

3. Ackerberg, D. and M. Botticini. 2002. "Endogenous Matching and the Empirical Determinants of Contract Form." Journal of Political Economy 110: 564-590.

4. Akanda, M., H. Isoda, and S. Ito. 2008. "Problem of Sharecrop Tenancy System in Rice Farming in Bangladesh: A Case Study on Alinapara Village in Sherpur District." Journal of International Farm Management 4: 23-30.

5. Alderman, H. and C. Paxson. 1992. "Do the Poor Insure? A Synthesis of the Literature on Risk and Consumption in Developing Countries." Economics in a Changing World 4: 49-78.

6. Ambrus, A., M. Mobius, and A. Szeidl. 2014. "Consumption Risk-sharing in Social Networks." American Economic Review 104(1): 149-182.

7. Antle, J. 1987. "Econometric Estimation of Producers' Risk Attitudes." American Journal of Agricultural Economics 69: 509-522.

8. Attanasio, O., A. Barr, J. Cardenas, G. Genicot, and C. Meghir. 2012. "Risk Pooling, Risk Preferences, and Social Networks." American Economic Journal: Applied Economics 4: 132-167.

9. Attanasio, O. and J. Rios-Rull. 2000. "Consumption Smoothing in Island Economies: Can Public Insurance Reduce Welfare?" European Economic Review 44: 1225-1258.

10. Banerjee, A. 1999. "Prospects and Strategies for Land Reforms." Annual World Bank Conference on Development Economics 1999, eds. B. Pleskovic and J. Stiglitz. 253-284.

11. Bardhan, P. 1980. "Interlocking Factor Markets and Agrarian Development: A Review of Issues." Oxford Econ. Papers 32: 82-98.

12. Basu, K. 1992. "Limited Liability and the Existence of Share Tenancy." Journal of Development Economics 38(1): 203-220.

13. Becker, G.S. 1973. "A Theory of Marriage: Part I." Journal of Political Economy 81: 813-846.

14. Bellemare, M. 2009. "Sharecropping, Insecure Land Rights and Land Titling Policies: A Case Study of Lac Alaotra, Madagascar." Development Policy Review 27: 87-106. 
15. Besley, T. 1995. "Savings, Credit, and Insurance." Handbook of Development Economics 3: 2123-2207.

16. Binswanger, H.P. 1980. "Attitudes towards Risk: Experimental Measurement in Rural India." American Journal of Agricultural Economics 62: 395-407.

17. Bloch, F., G. Genicot, and D. Ray. 2008. "Informal Insurance in Social Networks." Journal of Economic Theory 143(1): 36-58.

18. Bold, T. 2009. "Implications of Endogenous Group Formation for Efficient Risk-Sharing." The Economic Journal 119: 562-591.

19. Bramoulle, Y. and R. Kranton. 2007. "Risk Sharing Networks." J. Econ. Behavior and Organization 64: 275-294.

20. Cervantes-Godoy, D. and J. Dewbre. 2010. "Economic Importance of Agriculture for Poverty Reduction." OECD Food, Agriculture, and Fisheries Working Papers No. 23.

21. Chambers, C. and F. Echenique. "When Does Aggregation Reduce Risk Aversion?" Games and Economic Behavior (76) 2012 582-595.

22. Chiappori, P-A., K. Samphantharak, S. Schulhofer-Wohl, and R. Townsend. 2011. "Heterogeneity and Risk-Sharing in Village Economies." Working Paper.

23. Chiappori, P-A. and P. Reny. 2006. "Matching to share Risk." Working Paper.

24. Dawe, D. 2001. "How far down the path to free trade? The importance of rice price stabilization in developing Asia." Food Policy 26: 163-175.

25. Dercon, S. 1996. "Risk, Crop Choice, and Savings: Evidence from Tanzania." Economic Development and Cultural Change 44: 483-513.

26. Dercon, S. and P. Krishnan. 2000. "In Sickness and in Health: Risk Sharing within Households in Rural Ethiopia." Journal of Political Economy 108: 688-727.

27. Eeckhout, J. and K. Munshi. 2010. "Matching in Informal Financial Institutions." Journal of the European Economic Association 8: 947-988.

28. Fafchamps, M. 2008. "Risk-sharing Between Households." Handbook of Social Economics.

29. Fafchamps, M. and A. Lund. 2003. "Risk-sharing Networks in Rural Phillippines." Journal of Development Economics 71: 261-287.

30. Fafchamps, M. and F. Gubert. 2007. "The Formation of Risk-Sharing Networks." Journal of Development Economic 83: 326-350. 
31. Genicot, G. and D. Ray. 2003. "Group Formation in Risk-Sharing Arrangements." Review of Economic Studies 70: 87-113.

32. Gine, X., P. Jakiela, D. Karlan, and J. Morduch. 2010. "Microfinance Games." American Economic Journal: Applied Economics 2(3): 60-95.

33. Grinstead, C. and J. Snell. 1997. "Introduction to Probability." American Mathematical Society.

34. Harrison, G., S. Humphrey, and A. Verschoor. 2010. "Choice Under Uncertainty: Evidence from Ethiopia, India, and Uganda." The Economic Journal 120: 80-104.

35. Kaneko, M. 1982. "The Central Assignment Game and the Assignment Markets." Journal of Mathematical Economics 10: 205-232.

36. Knudsen, O. and J. Nash. 1990. "Domestic Price Stabilization Schemes in Developing Countries." Economic Development and Cultural Change 38: 539-558.

37. Kurosaki, T. 1999. "Risk-Sharing Arrangements and the Structure of Risk and Time Preferences: Theory and Evidence from Village India." Working Paper.

38. Legros, P. and A. Newman. 2007. "Beauty is a Beast, Frog is a Prince: Assortative Matching with Nontrasferabilities." Econometrica 75(4): 1073-1102.

39. Li, Sunxi, H. Sun, and P. Chen. 2012. "Assortative Matching of Risk-Averse Agents with Endogenous Risk." Journal of Economics (online only).

40. Ligon, E., J. Thomas, and T. Worrall. 2002. "Informal Insurance Arrangements with Limited Commitment: Theory and Evidence from Village Economies." Review of Economic Studies 69: 209-244.

41. Mazzocco, M. 2007. "Household intertemporal behavior: a collective characterization and a test of commitment." Review of Economic Studies 74: 857-895.

42. Meyer, J. 1987. "Two-Moment Decision Models and Expected Utility Maximization." American Economic Review 77(3):421-430.

43. Mobarak, A. M. and M. Rosenzweig. 2012. "Selling Formal Insurance to the Informally Insured." Working Paper.

44. Minot, N. 2010. "Food Price Stabilization: Lessons for Eastern and Southern Africa." Risk Management in African Agriculture Seminar Presentation.

45. Morduch, J. 1995. "Income Smoothing and Consumption Smoothing." J. Econ. Perspectives 9: 103-114. 
46. Rathore, V.S., J.P. Singh, and R.K. Beniwal. 2009. "Rainfed Farming Systems of Hyper Arid Northwestern Rajasthan: an Analysis." Annals of the Arid Zone 48: 125-131.

47. Rosenzweig, M. 1988. "Risk, Implicit Contracts, and the Family in Rural Areas of LowIncome Countries." Economic Journal 98: 1148-1170.

48. Rosenzweig, M. and H.P. Binswanger. 1993. "Wealth, Weather Risk, and the Composition and Profitability of Agricultural Investments." The Economic Journal 103: 56-78.

49. Rosenzweig, M. and O. Stark. 1989. "Consumption Smoothing, Migration, and Marriage: Evidence from Rural India." Journal of Political Economy 97: 905-926.

50. Schulhofer-Wohl, S. 2006. "Negative Assortative Matching of Risk-Averse Agents with Transferable Expected Utility." Economic Letters 92: 383-388.

51. Shapley, L. and M. Shubik. 1971. "The assignment game I: The Core." International Journal of Game Theory 1: 111-130.

52. Sharpe, William F. 1966. "Mutual Fund Performance". Journal of Business 39: 119-138.

53. Singh, H., G. Singh, J. Samuel, and R. Pathak. 2003. "Precision Farming in Horticulture." Proceedings of the National Seminar Workshop on Hi-Tech Horticulture and Precision Farming.

54. Stiglitz 1974. 1974. "Incentives and Risk Sharing in Sharecropping." Review of Economic Studies 41: 219-255.

55. Townsend, R. 1994. "Risk and Insurance in Village India." Econometrica 62(3): 539-591.

56. Townsend, R. and R. Mueller. 1998. "Mechanism Design and Village Economies: From Credit to Tenancy to Cropping Groups." Review of Economic Dyanmics 1: 119-172.

57. Wang, X. 2013a. "A Note on Moral Hazard and Linear Compensation Schemes." Working Paper.

58. Wang, X. 2013b. "Risk, Incentives, and Contracting Relationships." Working Paper.

59. Wang, X. 2015 (forthcoming). "Policymaking and the Adaptability of Informal Institutions." World Bank Economic Review.

60. Wilson, R. 1968. "The Theory of Syndicates." Econometrica 36: 119-132.

61. Wright, B. 2001. "Storage and Price Stabilization." Handbook of Agricultural Economics 1: 818-861. 


\section{Appendix}

\subsection{A.1: Equivalence of matching when choosing joint income distribution and when choosing individual income distribution and sharing the pooled re- turns}

This discussion clarifies the relationship between a model where matched individuals jointly choose which income distribution to face, and then share the realized return, versus a model where individuals choose their own income distribution, then match and share the pooled realized returns.

Suppose that, as in the benchmark model, a spectrum of projects $p \geq 0$ is available, where $Y_{p}=p+V(p)^{\frac{1}{2}} Y, E(Y)=0, V(Y)=1$, and $V(p)>0, V^{\prime}(p)>0$, and $V^{\prime \prime}(p)>0$. Each individual chooses a personal income distribution from the spectrum $Y_{p}$, and pools realized returns with her partner. An individual can always choose autarky if she prefers this to matching with any possible partner. A matched pair commits ex ante to a sharing rule contingent on pooled income, $s\left(Y_{p_{1}}+Y_{p_{2}}\right)$. (For ease of notation, let $Y_{k} \equiv Y_{p_{k}}$.)

Assume that incomes are correlated. Recall that $\operatorname{Var}\left(Y_{1}+Y_{2}\right)=\operatorname{Var}\left(Y_{1}\right)+\operatorname{Var}\left(Y_{2}\right)+$ $2 \operatorname{Cov}\left(Y_{1}, Y_{2}\right)$, where $\operatorname{Cov}\left(Y_{1}, Y_{2}\right)=E Y_{1} Y_{2}-E Y_{1} E Y_{2}=E Y_{1} Y_{2}-p_{1} p_{2}$.

Since $Y_{1}=p_{1}+V\left(p_{1}\right)^{\frac{1}{2}} Y$ and $Y_{2}=p_{2}+V\left(p_{2}\right)^{\frac{1}{2}} Y$ :

$$
\begin{aligned}
E\left(Y_{1} Y_{2}\right) & =E\left(p_{1} p_{2}+p_{1} V\left(p_{2}\right)^{\frac{1}{2}} Y+p_{2} V\left(p_{1}\right)^{\frac{1}{2}} Y+V\left(p_{1}\right)^{\frac{1}{2}} V\left(p_{2}\right)^{\frac{1}{2}} Y^{2}\right) \\
& =p_{1} p_{2}+E\left[V\left(p_{1}\right)^{\frac{1}{2}} V\left(p_{2}\right)^{\frac{1}{2}} Y^{2}\right]
\end{aligned}
$$

And:

$$
\begin{aligned}
E\left(Y^{2}\right) & =V(Y)-(E Y)^{2} \\
& =1
\end{aligned}
$$

So:

$$
\begin{aligned}
E\left(Y_{1} Y_{2}\right) & =p_{1} p_{2}+V\left(p_{1}\right)^{\frac{1}{2}} V\left(p_{2}\right)^{\frac{1}{2}} \\
\operatorname{Cov}\left(Y_{1}, Y_{2}\right) & =p_{1} p_{2}+V\left(p_{1}\right)^{\frac{1}{2}} V\left(p_{2}\right)^{\frac{1}{2}}-p_{1} p_{2} \\
\operatorname{Var}\left(Y_{1}+Y_{2}\right) & =V\left(p_{1}\right)+V\left(p_{2}\right)+2 V\left(p_{1}\right)^{\frac{1}{2}} V\left(p_{2}\right)^{\frac{1}{2}}
\end{aligned}
$$

Thus, members of a matched pair can choose individual income distributions $p_{1}$ and $p_{2}$ in a number of ways to achieve mean pooled income $p=p_{1}+p_{2}$ for any $p>0$. The optimal strategy for the partners in this model, given that they want expected joint income to be $p$, is to choose $p_{1}$ and $p_{2}$ to minimize the variance of joint income, given $p_{1}+p_{2}=p$ (Appendix 4 shows why this is the case). 
Solving:

$$
\begin{gathered}
\min _{p_{1}, p_{2}} V\left(p_{1}\right)+V\left(p_{2}\right)+2 V\left(p_{1}\right)^{\frac{1}{2}} V\left(p_{2}\right)^{\frac{1}{2}} \text { s.t. } \\
p_{1}+p_{2}=p
\end{gathered}
$$

it is straightforward to see that $p_{1}^{*}=p_{2}^{*}=\frac{p}{2}$, so that $V\left(p \mid p_{1}^{*}, p_{2}^{*}\right)=2 V\left(\frac{p}{2}\right)+4 V\left(\frac{p}{2}\right)^{\frac{1}{2}}$.

Hence, matched pairs choose from a frontier of joint income distributions described by: $Y_{p}=$ $p+\left[2 V\left(\frac{p}{2}\right)+4 V\left(\frac{p}{2}\right)^{\frac{1}{2}}\right]^{\frac{1}{2}} Y$, where $E(Y)=0, V(Y)=1$.

Thus, although individuals ostensibly choose own income in this model, they are actually choosing a distribution of pooled income as a matched pair, and each prefers her equilibrium outcome as a matched pair to her equilibrium outcome in autarky. Hence, this is substantively equivalent to the benchmark model, where matched pairs choose their joint income distribution, and each person is assumed to prefer being matched to being unmatched. I work with the joint choice model in this paper because it illustrates the dependence of the equilibrium matching on the trade-off between production and insurance more cleanly.

\subsection{A.2: Proof of Lemma 1 (NTU problem has TU representation)}

Lemma 1: Expected utility is transferable in this model. Denote a matched pair by $\left(r_{1}, r_{2}\right)$ and their chosen project by $p^{*}\left(r_{1}, r_{2}\right)$. Let $C E\left(r_{1}, r_{2}, p^{*}\left(r_{1}, r_{2}\right)\right)$ describe the certainty-equivalent of a matched pair in equilibrium; it is twice continuously differentiable in each argument. Then:

$$
\begin{aligned}
& \frac{\partial C E\left(r_{1}, r_{2}, p^{*}\left(r_{1}, r_{2}\right)\right)}{\partial r_{1} r_{2}}>0 \Leftrightarrow \text { unique PAM } \\
& \frac{\partial C E\left(r_{1}, r_{2}, p^{*}\left(r_{1}, r_{2}\right)\right)}{\partial r_{1} r_{2}}<0 \Leftrightarrow \text { unique } N A M
\end{aligned}
$$

The equilibrium matching maximizes the sum of certainty-equivalents, and is Pareto efficient.

Proof: The following program characterizes the optimal contract and project choice for a matched pair $\left(r_{1}, r_{2}\right)$ :

$$
\begin{gathered}
\max _{s\left(y_{p}\right)} \int_{-\infty}^{\infty}-e^{-r_{1}\left[y_{p}-s\left(y_{p}\right)\right]} f\left(y_{p} \mid p\right) d y_{p} \quad \text { s.t. } \\
\int_{-\infty}^{\infty}-e^{-r_{2} s\left(y_{p}\right)} f\left(y_{p} \mid p\right) d y_{p} \geq-e^{-v}
\end{gathered}
$$

An increase in $r_{2}$ 's expected utility strictly corresponds to a decrease in $r_{1}$ 's expected utility, since more output for $r_{2}$ means less output for $r_{1}$, so the constraint binds.

Solving shows that $r_{2}$ 's share of realized output $y_{p}$ is:

$$
s^{*}\left(y_{p} \mid p\right)=\frac{r_{1}}{r_{1}+r_{2}} y_{p}+\frac{1}{r_{2}} \log \int_{-\infty}^{\infty} e^{-\frac{r_{1} r_{2}}{r_{1}+r_{2}} y_{p}} f\left(y_{p} \mid p\right) d y_{p}+\frac{1}{r_{2}} v
$$


Plug this expression into $r_{1}$ 's objective function. Then the optimal project solves

$$
\max _{p \in \Pi}-e^{\frac{r_{1}}{r_{2}} v}\left[\int_{-\infty}^{\infty} e^{-\frac{r_{1} r_{2}}{r_{1}+r_{2}} y_{p}} f\left(y_{p} \mid p\right) d y_{p}\right]^{1+\frac{r_{1}}{r_{2}}}
$$

where $-e^{\frac{r_{1}}{r_{2}} v}$ is just a scaling factor.

So, we can write the indirect utility of an agent $r_{1}$ who ensures his partner, $r_{2}$, a level of expected utility $-e^{-v}$ :

$$
\phi\left(r_{1}, r_{2}, v\right)=-e^{\frac{r_{1}}{r_{2}} v}\left[\int_{-\infty}^{\infty} e^{-\frac{r_{1} r_{2}}{r_{1}+r_{2}} y_{p}} f\left(y_{p} \mid p\right) d y_{p}\right]^{1+\frac{r_{1}}{r_{2}}}
$$

Partner $r_{2}$ receives expected utility:

$$
\begin{aligned}
E U_{r_{2}}(v) & =-e^{-v} \\
& =-e^{-r_{2} \frac{v}{r_{2}}}
\end{aligned}
$$

The certainty-equivalent $C E_{i}$ for an individual $r_{i}$ facing risky income stream $Y_{i}$ is:

$$
\begin{aligned}
-e^{-r_{i} C E_{i}} & =E\left[-e^{-r_{i} Y_{i}}\right] \Rightarrow \\
C E_{i} & =-\frac{1}{r_{i}} \log E\left[e^{-r_{i} Y_{i}}\right]
\end{aligned}
$$

Clearly, the certainty-equivalent is a monotonic transformation of expected utility. Hence, showing that the slope of the Pareto frontier of certainty-equivalents is -1 shows expected utility is transferable.

The certainty-equivalent of each member is:

$$
\begin{aligned}
C E_{r_{1}}(v) & =-\left(\frac{1}{r_{1}}+\frac{1}{r_{2}}\right) \log \left[\int_{-\infty}^{\infty} e^{-\frac{r_{1} r_{2}}{r_{1}+r_{2}} Y_{p}} f\left(y_{p} \mid p^{*}\left(r_{1}, r_{2}\right)\right) d y_{p}\right]-\frac{v}{r_{2}} \\
C E_{r_{2}}(v) & =\frac{v}{r_{2}}
\end{aligned}
$$

There is clearly a one-to-one trade-off between the certainty-equivalent of $r_{1}$ and the certaintyequivalent of $r_{2}$. In other words, the slope of the Pareto frontier of expected utility (modulo a monotonic transformation) is -1 . Hence, expected utility is transferable.

The proof that the equilibrium matching maximizes the sum of certainty-equivalents and the sum of expected project returns, and is Pareto efficient, is straightforward. First, if a matching where each pair is engaged in their optimal project and sharing rule does not maximize the sum of certainty-equivalents, there would be at least one profitable blocking. Second, the sum of certaintyequivalents is a social welfare function, so as the matching maximizes this, it must be Pareto efficient. (Another way to see this is by recalling the no-blocking condition of the equilibrium.) 


\subsection{A.3: Differently-sized groups}

We know (see Appendix 2) that the sum of certainty-equivalents for a given pair $\left(r_{1}, r_{2}\right)$ with representative risk tolerance $R=\frac{1}{r_{1}}+\frac{1}{r_{2}}$ in the benchmark case is given by:

$$
\begin{aligned}
C E(R) & =-R \log K_{Y_{p^{*}(R)}} \\
& =p^{*}-\sum_{n=2}^{\infty} \frac{(-1)^{n}}{n ! R^{n-1}} V\left(p^{*}\right)^{\frac{n}{2}} k_{n}(y)
\end{aligned}
$$

Then:

$$
\begin{aligned}
\frac{\partial C E}{\partial R} & =\sum_{n=2}^{\infty} \frac{(-1)^{n}}{n !} \frac{(n-1)}{R^{n}} V\left(p^{*}\right)^{\frac{n}{2}} k_{n}(y) \\
\frac{\partial R}{\partial r_{2}} & =-\frac{1}{r_{2}^{2}}
\end{aligned}
$$

But we know that the risk premium for any risk-averse individual facing any risky project $p$ is positive:

$$
\begin{aligned}
R P(p) & =\sum_{n=2}^{\infty} \frac{(-1)^{n}}{n ! R^{n-1}} V(p)^{\frac{n}{2}} k_{n}(y)>0 \Leftrightarrow \\
\frac{1}{R} R P(p) & =\frac{1}{R} \sum_{n=2}^{\infty} \frac{(-1)^{n}}{n ! R^{n-1}} V(p)^{\frac{n}{2}} k_{n}(y)>0
\end{aligned}
$$

Hence:

$$
\begin{aligned}
\frac{\partial C E}{\partial R} & =\sum_{n=2}^{\infty} \frac{(-1)^{n}}{n !} \frac{(n-1)}{R^{n}} V\left(p^{*}\right)^{\frac{n}{2}} k_{n}(y) \\
& >\frac{1}{R} \sum_{n=2}^{\infty} \frac{(-1)^{n}}{n ! R^{n-1}} V(p)^{\frac{n}{2}} k_{n}(y) \\
& >0
\end{aligned}
$$

But:

$$
\begin{aligned}
\frac{\partial C E}{\partial r_{2}} & =\frac{\partial C E}{\partial R} \frac{\partial R}{\partial r_{2}} \\
& <0
\end{aligned}
$$

Hence, $C E\left(r_{1}, r_{2}\right)$ is decreasing in the risk aversion of any partner, since $C E(\cdot, \cdot)$ is symmetric in each of its arguments.

Thus, if $|G 1|<|G 2|$, for example, it is the most risk-averse excess agents of $G 2$ who will 
remain unmatched in equilibrium. Why? The following example best illustrates the intuition: let $G 1=\left\{r_{1}^{A}, r_{2}^{A}\right\}$ and $G 2=\left\{r_{1}^{B}, r_{2}^{B}, r_{3}^{B}\right\}$, where agents are ordered from least to most risk-averse. Suppose that it is not the most risk-averse agent who remains unmatched in equilibrium-suppose

$r_{2}^{B}$ is unmatched (which is the least favored option for all agents), while $\left(r_{1}^{A}, r_{1}^{B}\right)$ and $\left(r_{2}^{A}, r_{3}^{B}\right)$ are matched, and individual payoffs for the matched pairs are $\left(C E_{11}-v_{1}, v_{1}\right)$ and $\left(C E_{23}-v_{3}, v_{3}\right)$. But then $r_{2}^{B}$ can offer to match with $r_{2}^{A}$ and receive individual payoff $v_{3}$, which she prefers to being unmatched, and this leaves $r_{2}^{A}$ with $C E_{22}-v_{3}>C E_{23}-v_{3}$, since $C E\left(r_{1}, r_{2}\right)$ is decreasing in risk aversion as proved above. Thus, $r_{2}^{A}$ and $r_{2}^{B}$ will deviate and match with each other instead, meaning that $\left\{\left(r_{1}^{A}, r_{1}^{B}\right),\left(r_{2}^{A}, r_{3}^{B}\right)\right\}$ is not stable. The general point is that, because less risk-averse partners can always generate more surplus together, they can always find a division of the surplus which beats the best offer the most risk-averse agent can make. (Remember, the equilibrium matching pattern itself is determined by whether it's the less risk-averse agent or the more risk-averse agent who experiences a larger difference between matching with a less risk-averse versus a more riskaverse partner.)

Thus, in this simple example, it is $r_{3}^{B}$ who will be unmatched in equilibrium. Then the main matching results apply to the remaining agents, $G 1=\left\{r_{1}^{A}, r_{2}^{A}\right\}$ and $G 2=\left\{r_{1}^{B}, r_{2}^{B}\right\}$.

\subsection{A.4: Proof of Proposition 1 (main matching result) and Corollary 1 (coef- ficient of variation result)}

Proposition 1: Recall that $V(p)$ describes the variance cost of a project with mean return $p$. Then $M(p) \equiv V^{\prime}(p)$ describes the marginal variance cost of a project with mean return $p$.

(a) A sufficient condition for PAM to be the unique equilibrium match is $M^{\prime \prime}(p)<0$ for $p>0$ (concavity).

(b) A sufficient condition for $N A M$ to be the unique equilibrium match is $M^{\prime \prime}(p)>0$ for $p>0$ (convexity).

(c) A sufficient condition for any match to be sustainable as an equilibrium is $M^{\prime \prime}(p)=0$ for $p>0$ (linearity).

Proof: Suppose a matched pair $\left(r_{1}, r_{2}\right)$ has to choose from a family of income distributions defined by $Y_{p}=p+V(p)^{\frac{1}{2}} Y$, where $Y$ is a random variable with well-defined cumulants: $k_{1}(y)=$ $E(Y)=0, k_{2}(y)=V(Y)=1$, infinite support. (The cumulant-generating function of a random variable is the $\log$ of the moment-generating function; the $n^{\text {th }}$ order cumulant is the $n^{\text {th }}$ derivative of the cumulant-generating function evaluated at 0 .)

Recall that the representative risk tolerance of a matched group is the sum of the risk tolerances of the members. So, the representative risk tolerance of $\left(r_{1}, r_{2}\right)$ is $R=\frac{1}{r_{1}}+\frac{1}{r_{2}}$.

We know from Appendix 2 that the expected utility of a group with representative risk aversion $r$ (reciprocal of representative risk tolerance $R$ ) which has chosen a project $p$ with risky stream of returns $Y_{p}$ is: 


$$
\begin{aligned}
E\left[-e^{-r\left[p+V(p)^{\frac{1}{2}} Y\right]}\right] & =-e^{-r p} \int e^{-r V(p)^{\frac{1}{2}} y} f(y) d y \\
& =-\int e^{-\frac{1}{R}\left[p+V(p)^{\frac{1}{2}} y\right]} f(y) d y
\end{aligned}
$$

Divide by $(-1)$, transform by log, and multiply by $(-R)$ for the certainty-equivalent. Since we want to maximize expected utility, this implies we want to choose $p$ to maximize the certaintyequivalent:

$$
\begin{aligned}
C E(p ; R) & =-R \log E\left[e^{-\frac{1}{R}\left[p+V(p)^{\frac{1}{2}} Y\right]}\right] \\
& =-R\left[-\frac{1}{R} p+\log E\left[e^{-\frac{1}{R} V(p)^{\frac{1}{2}} Y}\right]\right] \\
& =p-R\left[\sum_{n=2}^{\infty} \frac{(-1)^{n}}{n ! R^{n}} V(p)^{\frac{n}{2}} k_{n}(y)\right] \\
& =p-\frac{1}{2 R} V(p)-\sum_{n=3}^{\infty} \frac{(-1)^{n}}{n ! R^{n-1}} V(p)^{\frac{n}{2}} k_{n}(y)
\end{aligned}
$$

Then:

$$
\frac{\partial C E}{\partial R}=\frac{1}{2 R^{2}} V\left(p^{*}\right)+\sum_{n=3} \frac{(-1)^{n}}{n !} \frac{(n-1)}{R^{n}} V\left(p^{*}\right)^{\frac{n}{2}} k_{n}(y)
$$

And:

$$
\begin{aligned}
\frac{\partial^{2} C E}{\partial R^{2}}= & -\frac{1}{R^{3}} V\left(p^{*}\right)+\frac{1}{2 R^{2}} V^{\prime}\left(p^{*}\right) \frac{\partial p^{*}}{\partial R}-\sum_{n=3} \frac{(-1)^{n}}{n !} \frac{n(n-1)}{R^{n+1}} V\left(p^{*}\right)^{\frac{n}{2}} k_{n}(y) \\
& +\sum_{n=3} \frac{(-1)^{n}}{n !} \frac{(n-1)}{R^{n}} \frac{n}{2} V\left(p^{*}\right)^{\frac{n}{2}-1} V^{\prime}\left(p^{*}\right) \frac{\partial p^{*}}{\partial R} k_{n}(y) \\
= & {\left[-\frac{2}{R} \frac{V\left(p^{*}\right)}{V^{\prime}\left(p^{*}\right)}+\frac{\partial p^{*}}{\partial R}\right]\left[\sum_{n=3} \frac{(-1)^{n}}{(n-2) !} \frac{1}{2 R^{n}} V\left(p^{*}\right)^{\frac{n}{2}-1} V^{\prime}\left(p^{*}\right) k_{n}(y)+\frac{1}{2 R^{2}} V^{\prime}\left(p^{*}\right)\right] }
\end{aligned}
$$

where we know this second bracketed term is positive because it is bounded below by $\frac{1}{R}>0$. We establish this bound by recalling the first-order condition of the optimization problem:

$$
F O C_{p}=0:-\frac{1}{R}+\frac{1}{2 R^{2}} V\left(p^{*}\right)+\sum_{n=3} \frac{(-1)^{n}}{(n-1) !} \frac{1}{2 R^{n}} V\left(p^{*}\right)^{\frac{n}{2}-1} V^{\prime}\left(p^{*}\right) k_{n}(y)=0
$$

This implies:

$$
\frac{1}{2 R^{2}} V\left(p^{*}\right)+\sum_{n=3} \frac{(-1)^{n}}{(n-1) !} \frac{1}{2 R^{n}} V\left(p^{*}\right)^{\frac{n}{2}-1} V^{\prime}\left(p^{*}\right) k_{n}(y)=\frac{1}{R}
$$


And the first term of the second bracketed expression:

$$
\sum_{n=3} \frac{(-1)^{n}}{(n-2) !} \frac{1}{2 R^{n}} V\left(p^{*}\right)^{\frac{n}{2}-1} V^{\prime}\left(p^{*}\right) k_{n}(y)>\sum_{n=3} \frac{(-1)^{n}}{(n-1) !} \frac{1}{2 R^{n}} V\left(p^{*}\right)^{\frac{n}{2}-1} V^{\prime}\left(p^{*}\right) k_{n}(y)
$$

Now, we seek conditions such that:

$$
\frac{\partial^{2} C E}{\partial R^{2}}>,<0
$$

Using the expression for $\frac{d^{2} C E}{d R^{2}}$, and the fact that the second bracketed expression is a positive constant, we know that:

$$
\begin{aligned}
\frac{\partial^{2} C E}{\partial R^{2}} & >,<0 \Leftrightarrow \\
\frac{\partial p^{*}}{\partial R} & >,<\frac{2}{R} \frac{V\left(p^{*}\right)}{V^{\prime}\left(p^{*}\right)}
\end{aligned}
$$

Now, find $\frac{\partial p^{*}}{\partial R}$ by implicitly differentiating $F O C_{p}=0$ :

$$
\begin{gathered}
\text { FOC }_{p}=0: \frac{1}{2 R^{2}} V\left(p^{*}\right)+\sum_{n=3} \frac{(-1)^{n}}{(n-1) !} \frac{1}{2 R^{n}} V\left(p^{*}\right)^{\frac{n}{2}-1} V^{\prime}\left(p^{*}\right) k_{n}(y)=\frac{1}{R} \\
\frac{\partial p^{*}}{\partial R}:-\frac{1}{R^{3}} V^{\prime}\left(p^{*}\right)+\frac{1}{2 R^{2}} V^{\prime \prime}\left(p^{*}\right) \frac{\partial p^{*}}{\partial R}-\sum_{n=3} \frac{(-1)^{n}}{(n-1) !} \frac{n}{R^{n+1}} \frac{1}{2} V\left(p^{*}\right)^{\frac{n}{2}-1} V^{\prime}\left(p^{*}\right) k_{n}(y) \\
+\sum_{n=3} \frac{(-1)^{n}}{(n-1) !} \frac{1}{R^{n}} \frac{1}{2}\left(\frac{n}{2}-1\right) V\left(p^{*}\right)^{\frac{n}{2}-2} V^{\prime}\left(p^{*}\right)^{2} k_{n} \frac{\partial p^{*}}{\partial R}+\sum_{n=3} \frac{(-1)^{n}}{(n-1) !} \frac{1}{R^{n}} \frac{1}{2} V\left(p^{*}\right)^{\frac{n}{2}-1} V^{\prime \prime}\left(p^{*}\right) k_{n}(y) \frac{\partial p^{*}}{\partial R}=-\frac{1}{R^{2}}
\end{gathered}
$$

Hence:

$$
\begin{gathered}
-V^{\prime}\left(p^{*}\right)+\frac{R}{2} V^{\prime \prime}\left(p^{*}\right) \frac{\partial p^{*}}{\partial R}+R^{2} \sum_{n=3} \frac{(-1)^{n}}{(n-1) !} \frac{1}{R^{n}} \frac{n}{2} V\left(p^{*}\right)^{\frac{n}{2}-2} V^{\prime}\left(p^{*}\right)^{2} k_{n}(y)\left[\frac{R}{2} \frac{\partial p^{*}}{\partial R}-\frac{V\left(p^{*}\right)}{V^{\prime}\left(p^{*}\right)}\right] \\
+R^{3} \sum_{n=3} \frac{(-1)^{n}}{(n-1) !} \frac{1}{R^{n}} \frac{1}{2} V\left(p^{*}\right)^{\frac{n}{2}-2} V^{\prime}\left(p^{*}\right) k_{n}(y) \frac{\partial p^{*}}{\partial R}\left[\frac{V\left(p^{*}\right) V^{\prime \prime}\left(p^{*}\right)}{V^{\prime}\left(p^{*}\right)}-V^{\prime}\left(p^{*}\right)\right]=-R
\end{gathered}
$$

This expression can be rearranged using the first-order condition:

$$
\begin{gathered}
-\frac{V^{\prime}\left(p^{*}\right)}{R}+\frac{\partial p^{*}}{\partial R}\left(\left[R-\frac{V^{\prime}\left(p^{*}\right)}{2}\right]\left[\frac{V^{\prime \prime}\left(p^{*}\right)}{V^{\prime}\left(p^{*}\right)}-\frac{V^{\prime}\left(p^{*}\right)}{V\left(p^{*}\right)}\right]+\frac{1}{2} V^{\prime \prime}\left(p^{*}\right)\right) \\
+\frac{R^{2}}{2} \frac{\partial p^{*}}{\partial R} \sum_{n=3} \frac{(-1)^{n}}{(n-1) !} \frac{1}{R^{n}} \frac{n}{2} V\left(p^{*}\right)^{\frac{n}{2}-2} V^{\prime}\left(p^{*}\right)^{2} k_{n}(y)-R \sum_{n=3} \frac{(-1)^{n}}{(n-1) !} \frac{1}{R^{n}} \frac{n}{2} V\left(p^{*}\right)^{\frac{n}{2}-1} V^{\prime}\left(p^{*}\right) k_{n}(y)=-1
\end{gathered}
$$


So we see that:

$$
\frac{\partial p^{*}}{\partial R}=\frac{\frac{V^{\prime}\left(p^{*}\right)}{R}-1+R \sum_{n=3} \frac{(-1)^{n}}{(n-1) !} \frac{1}{R^{n}} \frac{n}{2} V\left(p^{*}\right)^{\frac{n}{2}-1} V^{\prime}\left(p^{*}\right) k_{n}(y)}{R \frac{V^{\prime \prime}\left(p^{*}\right)}{V\left(p^{*}\right)}-R \frac{V^{\prime}\left(p^{*}\right)}{V\left(p^{*}\right)}+\frac{V^{\prime}\left(p^{*}\right)^{2}}{2 V\left(p^{*}\right)}+\frac{R^{2}}{2} \sum_{n=3} \frac{(-1)^{n}}{(n-1) !} \frac{1}{R^{n}} \frac{n}{2} V\left(p^{*}\right)^{\frac{n}{2}-2} V^{\prime}\left(p^{*}\right)^{2} k_{n}(y)}
$$

Hence:

$$
\begin{aligned}
\frac{\partial p^{*}}{\partial R} & >,<\frac{2}{R} \frac{V\left(p^{*}\right)}{V^{\prime}\left(p^{*}\right)} \Leftrightarrow \\
V^{\prime}\left(p^{*}\right)^{2} & >,<2 V\left(p^{*}\right) V^{\prime \prime}\left(p^{*}\right)
\end{aligned}
$$

Call the left-hand side of this inequality $L H S$, and the right-hand side, RHS. Observe that at $R=0$ (zero risk tolerance), $L H S=R H S=0$, since $p=0$ is the safest project available, so $p^{*}(0)=0$, and it is assumed that $V(0)=V^{\prime}(0)=0$.

So, compare $\frac{\partial L H S}{\partial R}$ and $\frac{\partial R H S}{\partial R}$ : both $L H S$ and $R H S$ are 0 at $R=0$. As $R$ increases, if $L H S$ either increases faster in $R$ or decreases slower than $R$ at every point $R$ in comparison to $R H S$, that is, $\frac{\partial L H S}{\partial R}>\frac{\partial R H S}{\partial R}$ for all $R>0$, then $\frac{\partial^{2} C E}{\partial R^{2}}>0$ since $L H S>R H S$ in the above inequality. And, vice versa, if $\frac{\partial L H S}{\partial R}<\frac{\partial R H S}{\partial R}$.

Thus:

$$
\begin{aligned}
V^{\prime}\left(p^{*}\right)^{2} & >,<2 V\left(p^{*}\right) V^{\prime \prime}\left(p^{*}\right) \Leftrightarrow \\
0 & >,<2 V\left(p^{*}\right) V^{\prime \prime \prime}\left(p^{*}\right) \frac{\partial p^{*}}{\partial R} \Leftrightarrow \\
0 & >,<V^{\prime \prime \prime}\left(p^{*}\right)
\end{aligned}
$$

where the last equivalency holds because $2 V\left(p^{*}\right) \frac{\partial p^{*}}{\partial R}>0$.

Hence, Proposition 1 is proven:

$$
\begin{aligned}
& V^{\prime \prime \prime}(p)<0 \forall p \geq 0 \Leftrightarrow \frac{\partial^{2} C E}{\partial R^{2}}>0 \forall R \geq 0 \Rightarrow \text { unique PAM } \\
& V^{\prime \prime \prime}(p)>0 \forall p \geq 0 \Leftrightarrow \frac{\partial^{2} C E}{\partial R^{2}}<0 \forall R \geq 0 \Rightarrow \text { unique } N A M
\end{aligned}
$$

Note that if our focus were not on identifying conditions for assortative matching that are independent of risk type distributions, we could easily write a necessary and sufficient condition for PAM and NAM. Instead of seeking conditions for global convexity and concavity of $C E(R)$ in $R$, the condition simply has to hold for each set of representative risk tolerances for every possible matching in the given population of risk types. 
A quick final note: we need $C E(p ; R)$ to be concave in $p$ for a well-defined maximum:

$$
\begin{aligned}
\frac{\partial^{2} C E}{\partial p^{2}} & =-\frac{1}{2 R} V^{\prime \prime}(p)-\sum_{n=3}^{\infty} \frac{(-1)^{n}}{n ! R^{n-1}} \frac{n}{2}\left[\left(\frac{n}{2}-1\right) V(p)^{\frac{n}{2}-2} V^{\prime}(p)^{2}+V(p)^{\frac{n}{2}-1} V^{\prime \prime}(p)\right] k_{n}(y) \\
& =-\frac{1}{2}\left[\frac{1}{R} V^{\prime \prime}(p)+\sum_{n=3}^{\infty} \frac{(-1)^{n-1}}{(n-1) ! R^{n-1}}\left[\frac{n}{2} V(p)^{\frac{n}{2}-2} V^{\prime}(p)^{2}+V(p)^{\frac{n}{2}-2}\left(V(p) V^{\prime \prime}(p)-V^{\prime}(p)^{2}\right)\right]\right] \\
& <0
\end{aligned}
$$

Corollary 1: Let $C V(p)=\frac{V(p)^{\frac{1}{2}}}{p}$ denote the ratio of a project's mean return to its standard deviation for $p>0$. Then:

(a) $C V^{\prime}(p)<0 \forall p>0$ iff $M^{\prime \prime}(p)<0 \forall p>0(P A M)$

(b) $C V^{\prime}(p)>0 \forall p>0$ iff $M^{\prime \prime}(p)>0 \forall p>0(N A M)$

Proof: Recall that the Sharpe ratio is the reciprocal of the coefficient of variation: $S R(p) \equiv$ $\frac{1}{C V(p)}$. Thus, proving that the Sharpe ratio monotonically increases (decreases) in mean return iff the marginal variance cost is concave (convex) in mean return proves the corollary.

$$
\begin{aligned}
S R(p) & =\frac{p}{V(p)^{\frac{1}{2}}} \\
S R^{\prime}(p) & =\frac{V(p)^{\frac{1}{2}}-p \frac{1}{2} V(p)^{-\frac{1}{2}} V^{\prime}(p)}{V(p)}
\end{aligned}
$$

Then:

$$
\begin{aligned}
S R^{\prime}(p) & >\quad,<0 \Leftrightarrow V(p)^{\frac{1}{2}}>,<p \frac{1}{2} V(p)^{-\frac{1}{2}} V^{\prime}(p)[\text { since } V(p)>0 \forall p] \\
& \Leftrightarrow V(p)>,<\frac{1}{2} p V^{\prime}(p)
\end{aligned}
$$

Then note that $\operatorname{LHS}(p=0)=V(0)=0=\frac{1}{2} * 0 * V^{\prime}(0)=R H S(p=0)$. So, look at derivatives of LHS and RHS, since the domain is $p \geq 0$.

$$
V^{\prime}(p)>,<p V^{\prime \prime}(p)
$$

But we don't know under what conditions this inequality holds. So we again note that $L H S(p=$ $0)=V^{\prime}(0)=0=0 * V^{\prime \prime}(0)=R H S(p=0)$, and differentiate LHS, RHS again:

$$
\begin{aligned}
V^{\prime \prime}(p) & >,<V^{\prime \prime}(p)+p V^{\prime \prime \prime}(p) \Leftrightarrow \\
0 & >,<p V^{\prime \prime \prime}(p)
\end{aligned}
$$


Since the domain is $p \geq 0$, this means that:

$$
\begin{aligned}
V^{\prime \prime \prime}(p) & >0 \Leftrightarrow S R^{\prime}(p)<0 \forall p \geq 0 \\
& \Leftrightarrow ! N A M \\
& \Leftrightarrow C V^{\prime}(p)>0 \\
& \Leftrightarrow \frac{\partial^{2} p^{*}}{\partial R^{2}}<0
\end{aligned}
$$

and:

$$
\begin{aligned}
V^{\prime \prime \prime}(p) & <0 \Leftrightarrow S R^{\prime}(p)>0 \forall p \geq 0 \\
& \Leftrightarrow ! P A M \\
& \Leftrightarrow C V^{\prime}(p)<0 \\
& \Leftrightarrow \frac{\partial^{2} p^{*}}{\partial R^{2}}>0
\end{aligned}
$$

\subsection{A.5: Proof of Proposition 2 (NAM when income-smoothing is shut down)}

Proposition 2: Suppose that all agents in $G 1$ draw income iid from a distribution $M$, and all agents in $G 2$ draw income iid from a distribution $W$, so that all possible pairs face the same joint income distribution. Once matched, agents can commit ex ante to a return-contingent sharing rule. Then the unique equilibrium matching is NAM.

Proof: Suppose that all pairs must undertake the same project, $p$. For instance, the government mandates that all farmers must grow rice. This effectively shuts down the income-smoothing channel.

Differentiate $C E\left(r_{1}, r_{2}\right)$ with respect to $r_{1}$ and $r_{2}$ when there is no project choice, so that all pairs $\left(r_{1}, r_{2}\right)$ face the same risky income stream $f\left(Y_{p} \mid p\right)$. The cross-partial $\frac{d^{2} C E\left(r_{1}, r_{2}\right)}{d r_{1} d r_{2}}$ is:

$$
-\frac{r_{1} r_{2}}{\left(r_{1}+r_{2}\right)^{3}} \frac{\int e^{-\frac{r_{1} r_{2}}{r_{1}+r_{2}} y_{p}} f\left(y_{p} \mid p\right) d y_{p} \int y_{p}^{2} e^{-\frac{r_{1} r_{2}}{r_{1}+r_{2}} y_{p}} f\left(y_{p} \mid p\right) d y_{p}-\left[\int y_{p} e^{-\frac{r_{1} r_{2}}{r_{1}+r_{2}} y_{p}} f\left(y_{p} \mid p\right) d y_{p}\right]^{2}}{\left[\int e^{-\frac{r_{1} r_{2}}{r_{1}+r_{2}} y_{p}} f\left(y_{p} \mid p\right) d y_{p}\right]^{2}}
$$

But we know that:

$$
\int e^{-\frac{r_{1} r_{2}}{r_{1}+r_{2}} y_{p}} f\left(y_{p} \mid p\right) d y_{p} \int y_{p}^{2} e^{-\frac{r_{1} r_{2}}{r_{1}+r_{2}} y_{p}} f\left(y_{p} \mid p\right) d y_{p}>\left[\int y_{p} e^{-\frac{r_{1} r_{2}}{r_{1}+r_{2}} y_{p}} f\left(y_{p} \mid p\right) d y_{p}\right]^{2}
$$

since we know variance is always positive. Therefore:

$$
\int f\left(y_{p} \mid p\right) d y_{p} \int y_{p}^{2} f\left(y_{p} \mid p\right) d y_{p}>\left[\int y_{p} f\left(y_{p} \mid p\right) d y_{p}\right]^{2}
$$

and $g\left(Y_{p}\right)=e^{-\frac{r_{1} r_{2}}{r_{1}+r_{2}} Y_{p}}$ is a convex function. 
Hence:

$$
\frac{\partial^{2} C E\left(r_{1}, r_{2}\right)}{\partial r_{1} \partial r_{2}}<0
$$

and negative assortative matching therefore results as the unique equilibrium.

Chiappori and Reny (2006) and Schulhofer-Wohl (2006) also find that the unique matching pattern is negative-assortative when heterogenously risk-averse agents who are matched can commit ex ante to a return-contingent sharing rule of a given risk, but cannot choose what risk to face.

\subsection{A.6: Proof of Proposition 3 (PAM when consumption-smoothing is shut down)}

Proposition 3: Suppose that the slope of the sharing rule $s\left(R_{p}\right)=a+b R_{p}$ is fixed. For example, a wage law requires a 50-50 split of output. However, a matched pair is able to choose a risky project $p$, where $Y_{p}=p+V(p)^{\frac{1}{2}} Y$ as in the benchmark model. Then the unique equilibrium matching is PAM.

Proof: We know from the main matching result that the optimal sharing rule is linear. Suppose the slope of the sharing rule $s\left(Y_{p}\right)=a+b Y_{p}$ is fixed at $b$ for all possible pairs of risk types. (For example, the government mandates an equal split of the output, but is unable to prevent partners from making fixed transfers to one another.) Recall from the set up that $Y_{p}=p+V(p)^{\frac{1}{2}} Y$, $E(Y)=0, V(Y)=1$.

Fixing $b$ removes consumption-smoothing as an effective risk management tool (since transfers can no longer be conditioned on the realized return), leaving only income-smoothing (project choice). What happens to equilibrium risk-sharing relationships?

A matched pair $\left(r_{1}, r_{2}\right)$ chooses the relationship-specific transfer $a$ and project $p$ :

$$
\begin{gathered}
\max _{a, p} \int-e^{-r_{1}\left[-a+(1-b) y_{p}\right]} f\left(y_{p} \mid p\right) d y_{p} \text { s.t. } \\
\int-e^{-r_{2}\left[a+b y_{p}\right]} f\left(y_{p} \mid p\right) d y_{p} \geq-e^{-v}
\end{gathered}
$$

Using the structure on $Y_{p}$ :

$$
\begin{gathered}
\max _{a, p} \int-e^{-r_{1}\left[-a+(1-b)\left(p+V(p)^{\frac{1}{2}} y\right)\right]} f(y) d y \text { s.t. } \\
\int-e^{-r_{2}\left[a+b\left(p+V(p)^{\frac{1}{2}} y\right)\right]} f(y) d y \geq-e^{-v}
\end{gathered}
$$

The transfer $a$ is chosen to satisfy the constraint (which clearly binds in equilibrium), since the division of output is fixed at $b$ by the government. Basically, the transfer $a$ that $r_{1}$ must make to a partner $r_{2}$ to ensure her each level of expected utility $v$ measures the value of that relationship. 
Therefore:

$$
\begin{aligned}
\int e^{-r_{2}\left[a+b\left(p+V(p)^{\frac{1}{2}} y\right)\right]} f(y) d y & =e^{-v} \Leftrightarrow \\
e^{-r_{2} a} \int e^{-r_{2} b\left(p+V(p)^{\frac{1}{2}} y\right)} f(y) d y & =e^{-v} \Leftrightarrow \\
-\frac{1}{r_{2}}\left[-v+r_{2} b p-\log \int e^{-r_{2} b V(p)^{\frac{1}{2}} y} f(y) d y\right] & =a
\end{aligned}
$$

Then the equilibrium project selected is:

$$
\begin{aligned}
& p^{*}\left(r_{1}, r_{2}\right)=\arg \max _{\tilde{p} \in \Pi} \int-e^{-r_{1}\left[\frac{1}{r_{2}}\left[-v+r_{2} b \tilde{p}-\log \int e^{-r_{2} b V(\tilde{p})^{\frac{1}{2}} y} f(y) d y\right]+(1-b)\left(\tilde{p}+V(\tilde{p})^{\frac{1}{2}} y\right]\right.} f(y) d y \\
& =\arg \max _{\tilde{p} \in \Pi}-e^{\frac{r_{1}}{r_{2}} v-r_{1} \tilde{p}+\frac{r_{1}}{r_{2}} \log \int e^{-r_{2} b V(\tilde{p})^{\frac{1}{2}}} y} f(y) d y \int e^{-r_{1}(1-b) V(\tilde{p})^{\frac{1}{2}} y} f(y) d y
\end{aligned}
$$

Transform the objective function by dividing by -1 and taking logs:

$$
\max _{\tilde{p} \in \Pi} \frac{r_{1}}{r_{2}} v-r_{1} \tilde{p}+\frac{r_{1}}{r_{2}} \log \int e^{-r_{2} b V(\tilde{p})^{\frac{1}{2}} y} f(y) d y+\log \int e^{-r_{1}(1-b) V(\tilde{p})^{\frac{1}{2}} y} f(y) d y
$$

Then the first-order condition characterizing equilibrium project choice is:

$$
-1-\frac{b \frac{1}{2} V\left(p^{*}\right)^{\frac{1}{2}} V^{\prime}\left(p^{*}\right) \int y e^{-r_{2} b V(\tilde{p})^{\frac{1}{2}} y} f(y) d y}{\int e^{-r_{2} b V(\tilde{p})^{\frac{1}{2}}} y(y) d y}-\frac{(1-b) \frac{1}{2} V\left(p^{*}\right)^{\frac{1}{2}} V^{\prime}\left(p^{*}\right) \int y e^{-r_{1}(1-b) V(\tilde{p})^{\frac{1}{2}} y} f(y) d y}{\int e^{-r_{1}(1-b) V(\tilde{p})^{\frac{1}{2}} y} f(y) d y}=0
$$

Then the certainty-equivalent of $r_{1}$ is:

$$
\begin{aligned}
-e^{-r_{1} C E} & =-e^{\frac{r_{1}}{r_{2}} v-r_{1} p^{*}+\frac{r_{1}}{r_{2}} \log \int e^{-r_{2} b V\left(p^{*}\right)^{\frac{1}{2}} y} f(y) d y} \int e^{-r_{1}(1-b) V\left(p^{*}\right)^{\frac{1}{2}} y} f(y) d y \Leftrightarrow \\
C E_{r_{1}} & =-\frac{1}{r_{2}} v+p^{*}-\frac{1}{r_{2}} \log \int e^{-r_{2} b V\left(p^{*}\right)^{\frac{1}{2}} y} f(y) d y-\frac{1}{r_{1}} \log \int e^{-r_{1}(1-b) V\left(p^{*}\right)^{\frac{1}{2}} y} f(y) d y
\end{aligned}
$$

Then the sum of certainty-equivalents for $\left(r_{1}, r_{2}\right)$ is:

$$
C E\left(r_{1}, r_{2}\right)=p^{*}-\frac{1}{r_{2}} \log \int e^{-r_{2} b V\left(p^{*}\right)^{\frac{1}{2}} y} f(y) d y-\frac{1}{r_{1}} \log \int e^{-r_{1}(1-b) V\left(p^{*}\right)^{\frac{1}{2}} y} f(y) d y
$$


Using the first-order condition:

$$
\begin{aligned}
\frac{\partial C E}{\partial r_{1}}= & \frac{\partial p^{*}}{\partial r_{1}}-\frac{1}{r_{2}} \frac{-r_{2} b \frac{1}{2} V\left(p^{*}\right)^{\frac{1}{2}} V^{\prime}\left(p^{*}\right) \frac{\partial p^{*}}{\partial r_{1}} \int y e^{-r_{2} b V\left(p^{*}\right)^{\frac{1}{2}} y} f(y) d y}{\int e^{-r_{2} b V\left(p^{*}\right)^{\frac{1}{2}} y} f(y) d y}+\frac{1}{r_{1}^{2}} \log \int e^{-r_{1}(1-b) V\left(p^{*}\right)^{\frac{1}{2}} y} f(y) d y \\
& -\frac{1}{r_{1}} \frac{\left[-(1-b) V\left(p^{*}\right)^{\frac{1}{2}}-r_{1}(1-b) \frac{1}{2} V\left(p^{*}\right)^{\frac{1}{2}} V^{\prime}\left(p^{*}\right) \frac{\partial p^{*}}{\partial r_{1}}\right] \int y e^{-r_{1}(1-b) V\left(p^{*}\right)^{\frac{1}{2}} y} f(y) d y}{\int e^{-r_{1}(1-b) V\left(p^{*}\right)^{\frac{1}{2}} y} f(y) d y} \\
= & \frac{1}{r_{1}^{2}} \log \int e^{-r_{1}(1-b) V\left(p^{*}\right)^{\frac{1}{2}} y} f(y) d y+\frac{(1-b) V\left(p^{*}\right)^{\frac{1}{2}}}{r_{1}} \frac{\int y e^{-r_{1}(1-b) V\left(p^{*}\right)^{\frac{1}{2}} y} f(y) d y}{\int e^{-r_{1}(1-b) V\left(p^{*}\right)^{\frac{1}{2}} y} f(y) d y}
\end{aligned}
$$

And:

$$
\begin{gathered}
\frac{\partial C E}{\partial r_{2} \partial r_{1}}=-\frac{(1-b)}{r_{1}} \frac{1}{2} V\left(p^{*}\right)^{-\frac{1}{2}} V^{\prime}\left(p^{*}\right) \frac{\partial p^{*}}{\partial r_{2}} \frac{\int y e^{-r_{1}(1-b) V\left(p^{*}\right)^{\frac{1}{2}} y} f(y) d y}{\int e^{-r_{1}(1-b) V\left(p^{*}\right)^{\frac{1}{2}} y} f(y) d y} \\
+\frac{(1-b)}{r_{1}} \frac{1}{2} V\left(p^{*}\right)^{-\frac{1}{2}} V^{\prime}\left(p^{*}\right) \frac{\partial p^{*}}{\partial r_{2}} \frac{\int y e^{-r_{1}(1-b) V\left(p^{*}\right)^{\frac{1}{2}} y} f(y) d y}{\int e^{-r_{1}(1-b) V\left(p^{*}\right)^{\frac{1}{2}} y} f(y) d y} \\
-\frac{1}{2}(1-b)^{2} V^{\prime}\left(p^{*}\right) \frac{\partial p^{*}}{\partial r_{2}} \frac{\int e^{-r_{1}(1-b) V\left(p^{*}\right)^{\frac{1}{2}} y} f(y) d y \int y^{2} e^{-r_{1}(1-b) V\left(p^{*}\right)^{\frac{1}{2}} y} f(y) d y-\left[\int y e^{-r_{1}(1-b) V\left(p^{*}\right)^{\frac{1}{2}} y} f(y) d y\right]^{2}}{\left[\int e^{-r_{1}(1-b) V\left(p^{*}\right)^{\frac{1}{2}} y} f(y) d y\right]^{2}} \\
=-\frac{1}{2}(1-b)^{2} V^{\prime}\left(p^{*}\right) \frac{\partial p^{*}}{\partial r_{2}} \frac{\int e^{-r_{1}(1-b) V\left(p^{*}\right)^{\frac{1}{2}} y} f(y) d y \int y^{2} e^{-r_{1}(1-b) V\left(p^{*}\right)^{\frac{1}{2}} y} f(y) d y-\left[\int y e^{-r_{1}(1-b) V\left(p^{*}\right)^{\frac{1}{2}} y} f(y) d y\right]^{2}}{\left[\int e^{-r_{1}(1-b) V\left(p^{*}\right)^{\frac{1}{2}} y} f(y) d y\right]^{2}} \\
>0
\end{gathered}
$$

since $\frac{\partial p^{*}}{\partial r_{2}}<0$ and the fraction term is positive. Since the variance of $Y$ is positive:

$$
\int f(y) d y \int y^{2} f(y) d y>\left[\int y f(y) d y\right]^{2}
$$

and $g(y)=e^{-r_{1}(1-b) V\left(p^{*}\right)^{\frac{1}{2}} y}$ is a convex function.

Hence, positive assortative matching arises as the unique equilibrium.

So, if the government regulates wages by fixing the slope of the sharing rule at some $b \in[0,1]$, where pairs can still make within-pair state-independent transfers, the unique equilibrium matching pattern is always positive assortative, verifying our intuition that, because consumption-smoothing is held fixed, the "similarity of decisionmaking framework" dominates and people match with people who are like them because they will agree about project choice. This can be thought of as the counterpoint to shutting down the income-smoothing channel.

What are some implications of this understanding? The government may be motivated by equality concerns to specify an equal division of output in every relationship, but this may actually 
generate even more inequality by weakening the informal risk-management toolkit available to individuals, which then triggers endogenous change in risk-sharing networks. Specifically, if agents had been matched negative assortatively in the status quo (because the "cost function" of project mean is quite convex, say), then this imposition of wage equality leads to positive assortative matching, which may actually exacerbate inequality: there is a bigger spread in projects, with less risk-averse agents on projects with much higher expected returns while more risk-averse agents are on projects with much smaller expected returns, and less risk-averse agents abandon their roles as informal insurers, and more risk-averse agents wind up bearing more risk.

\subsection{A.7: Proof of Proposition 4 (mean incomes and risk tolerance)}

Proposition 4: Let $p^{*}\left(r_{i}, \mu\left(r_{i}\right)\right)$ denote the mean return of the project chosen by a matched pair $\left(r_{i}, \mu\left(r_{i}\right)\right)$. Then:

(a) If $M^{\prime \prime}(p)<0$ for $p>0$, then $p^{*}\left(r_{i}, \mu\left(r_{i}\right)\right)$ is convex in representative risk tolerance $R\left(r_{i}, \mu\left(r_{i}\right)\right)$.

(b) If $M^{\prime \prime}(p)>0$ for $p>0$, then $p^{*}\left(r_{i}, \mu\left(r_{i}\right)\right)$ is concave in representative risk tolerance $R\left(r_{i}, \mu\left(r_{i}\right)\right)$.

Proof: We want to show that the condition for global convexity and concavity of the certaintyequivalent of a group with representative risk tolerance $R$ in $R$ is equivalent to global convexity and concavity of the mean returns of projects chosen by matched pairs in equilibrium in the representative risk tolerances of those matched pairs.

We know from Appendix 4 that:

$$
\begin{aligned}
\frac{\partial^{2} C E}{\partial R^{2}} & >,<0 \forall R>0 \Leftrightarrow \\
\frac{\partial p^{*}}{\partial R} & >,<\frac{2}{R} \frac{V\left(p^{*}\right)}{V^{\prime}\left(p^{*}\right)} \forall R>0
\end{aligned}
$$

At $R=0, L H S=R H S=0$. So, find conditions under which $\frac{\partial L H S}{\partial R}>\frac{\partial R H S}{\partial R}$, and vice versa. Then:

$$
\begin{array}{r}
\frac{\partial L H S}{\partial R}-\frac{\partial R H S}{\partial R}>,<0 \Leftrightarrow \\
\frac{R}{2} V^{\prime}\left(p^{*}\right) \frac{\partial^{2} p^{*}}{\partial R^{2}}+\frac{\partial p^{*}}{\partial R}\left[\frac{R}{2} V^{\prime \prime}\left(p^{*}\right) \frac{\partial p^{*}}{\partial R}-\frac{1}{2} V^{\prime}\left(p^{*}\right)\right]>,<0
\end{array}
$$

The second term is positive or negative depending on whether the bracketed term is positive or negative:

$$
\begin{aligned}
\frac{\partial p^{*}}{\partial R}\left[\frac{R}{2} V^{\prime \prime}\left(p^{*}\right) \frac{\partial p^{*}}{\partial R}-\frac{1}{2} V^{\prime}\left(p^{*}\right)\right] & >,<0 \Leftrightarrow \\
\frac{\partial p^{*}}{\partial R} & >,<\frac{1}{R} \frac{V^{\prime}\left(p^{*}\right)}{V^{\prime \prime}\left(p^{*}\right)}
\end{aligned}
$$


Using the expression for $\frac{\partial p^{*}}{\partial R}$ we characterized by implicitly differentiating the first-order optimality condition in Appendix 4, we see that this inequality is equivalent to:

$$
2 R^{2}\left[\frac{1}{2} \sum_{n=3} \frac{(-1)^{n}}{(n-1) !} \frac{1}{R^{n}} \frac{n}{2} V\left(p^{*}\right)^{\frac{n}{2}-1} V^{\prime}\left(p^{*}\right) k_{n}(y)+\frac{1}{2 R^{2}} V^{\prime}\left(p^{*}\right)-\frac{1}{R}\right]\left[V^{\prime \prime}\left(p^{*}\right)-\frac{1}{2} \frac{V^{\prime}\left(p^{*}\right)^{2}}{V\left(p^{*}\right)}\right]>,<0
$$

But we know that the first bracketed term is positive, since:

$$
\begin{aligned}
\frac{1}{2} \sum_{n=3} \frac{(-1)^{n}}{(n-1) !} \frac{1}{R^{n}} \frac{n}{2} V\left(p^{*}\right)^{\frac{n}{2}-1} V^{\prime}\left(p^{*}\right) k_{n}(y) & >\frac{1}{2} \sum_{n=3} \frac{(-1)^{n}}{(n-1) !} \frac{1}{R^{n}} V\left(p^{*}\right)^{\frac{n}{2}-1} V^{\prime}\left(p^{*}\right) k_{n}(y) \\
& =\frac{1}{2 R^{2}} V^{\prime}\left(p^{*}\right)-\frac{1}{R}
\end{aligned}
$$

where the equality holds from $F O C_{p}=0$.

Hence:

$$
\begin{gathered}
\frac{\partial p^{*}}{\partial R}>,<\frac{1}{R} \frac{V^{\prime}\left(p^{*}\right)}{V^{\prime \prime}\left(p^{*}\right)} \Leftrightarrow \\
V^{\prime \prime}\left(p^{*}\right)-\frac{1}{2} \frac{V^{\prime}\left(p^{*}\right)^{2}}{V\left(p^{*}\right)}>,<0 \Leftrightarrow \\
2 V\left(p^{*}\right) V^{\prime \prime}\left(p^{*}\right)>,<V^{\prime}\left(p^{*}\right)^{2}
\end{gathered}
$$

But we know from Appendix 4 that:

$$
\begin{gathered}
2 V\left(p^{*}\right) V^{\prime \prime}\left(p^{*}\right)>,<V^{\prime}\left(p^{*}\right)^{2} \Leftrightarrow \\
V^{\prime \prime \prime}\left(p^{*}\right)>,<0 \Leftrightarrow \\
\frac{\partial^{2} C E}{\partial R^{2}}<,>0 \Leftrightarrow \\
\frac{R}{2} V^{\prime}\left(p^{*}\right) \frac{\partial^{2} p^{*}}{\partial R^{2}}+\frac{\partial p^{*}}{\partial R}\left[\frac{R}{2} V^{\prime \prime}\left(p^{*}\right) \frac{\partial p^{*}}{\partial R}-\frac{1}{2} V^{\prime}\left(p^{*}\right)\right]>,<0
\end{gathered}
$$

Hence:

$$
\begin{aligned}
V^{\prime \prime \prime}\left(p^{*}\right) & >,<0 \forall p>0 \Leftrightarrow \\
\frac{\partial p^{*}}{\partial R}\left[\frac{R}{2} V^{\prime \prime}\left(p^{*}\right) \frac{\partial p^{*}}{\partial R}-\frac{1}{2} V^{\prime}\left(p^{*}\right)\right] & >,<0 \text { and } \frac{\partial^{2} C E}{\partial R^{2}}<,>0 \forall R>0 \Leftrightarrow \\
\frac{R}{2} V^{\prime}\left(p^{*}\right) \frac{\partial^{2} p^{*}}{\partial R^{2}} & <,>0 \forall R>0 \Leftrightarrow \\
\frac{\partial^{2} p^{*}}{\partial R^{2}} & <,>0 \forall R>0
\end{aligned}
$$

Thus, while $V^{\prime \prime \prime}(p)$ (or any higher-order characteristics of the distributions of income streams) might be difficult to measure or estimate in practice, this result suggests a more empirically feasible approach: the mean incomes of matched groups in equilibrium are convex or concave in their 
representative risk tolerance if and only if $V^{\prime \prime \prime}(p)$ is negative or positive.

\subsection{A.8: Proof of Proposition 5 (endogenous group size)}

Proposition 5: Let $M(p) \equiv V^{\prime}(p)$ describe the marginal variance cost of each project $p$. Then:

(a) If $M^{\prime \prime}(p)<0$, the unique equilibrium is maximal-connectedness: the whole group, $G$, is matched.

(b) If $M^{\prime \prime}(p)>0$, the unique equilibrium is minimal-connectedness. Individuals match in negative assortative pairs: the $i^{\text {th }}$ least risk-averse person in $G$ is matched with the $i^{\text {th }}$ most risk-averse. (If $|G|$ is odd, then the most risk-averse individual in the population remains unmatched.)

Proof: We know from the proof of Proposition 1 that:

$$
\begin{aligned}
& M^{\prime \prime}(p)>0 \Leftrightarrow \frac{\partial^{2} C E}{\partial R^{2}}<0 \\
& M^{\prime \prime}(p)<0 \Leftrightarrow \frac{\partial^{2} C E}{\partial R^{2}}>0
\end{aligned}
$$

Recall the following property of a function $f: \mathbb{R}_{0}^{+} \rightarrow \mathbb{R}$ :

$$
\begin{aligned}
& f(0) \geq 0 \text { and } f(\cdot) \text { concave } \Rightarrow \forall x, y \in \mathbb{R}_{0}^{+}, f(x+y)<f(x)+f(y) \\
& f(0) \leq 0 \text { and } f(\cdot) \text { convex } \Rightarrow \forall x, y \in \mathbb{R}_{0}^{+}, f(x+y)>f(x)+f(y)
\end{aligned}
$$

That is, given $f(0) \geq 0$, a concave function is subadditive, and given $f(0) \leq 0$, a convex function is superadditive.

Recall that $C E(\cdot)$ is a function of the risk tolerance $R$ of a matched group. Since $C E(0)=0$, we know that:

$$
\begin{aligned}
& M^{\prime \prime}(p)>0 \forall p \geq 0 \Leftrightarrow C E\left(R_{1}+R_{2}\right)<C E\left(R_{1}\right)+C E\left(R_{2}\right) \forall R_{1}, R_{2} \in \mathbb{R}_{0}^{+} \\
& M^{\prime \prime}(p)<0 \forall p \geq 0 \Leftrightarrow C E\left(R_{1}+R_{2}\right)>C E\left(R_{1}\right)+C E\left(R_{2}\right) \forall R_{1}, R_{2} \in \mathbb{R}_{0}^{+}
\end{aligned}
$$

But representative risk tolerance is additive: the representative risk tolerance of a matched group is the sum of the individual risk tolerances. This implies that, when $M^{\prime \prime}(p)>0$ and $C E(\cdot)$ is concave in risk tolerance $R$, the matching that maximizes the total sum of certainty-equivalents is unique and is the minimal matching-that is, individuals match in partnerships (since production requires at least two collaborators), and we know from Proposition 1 that these partnerships will be negative assortative (and, from Appendix 3, that if there is an odd number of people in the population, the most risk-averse individual will remain unmatched). And, when $M^{\prime \prime}(p)<0$ and $C E(\cdot)$ is convex in risk tolerance $R$, the matching that maximizes the total sum of certaintyequivalents is unique and is the maximal matching-that is, all individuals match in one big group. 


\subsection{A.9: CARA Preferences and the Two Moment Result}

The fact that equilibrium assortative matching is determined by the first two moments is not a consequence of CARA preferences; rather, it is a consequence of the location-scale property of the return distributions (see Meyer (1987) for a detailed discussion of the location-scale family). CARA preferences are associated with reduction to two moments because they are commonly paired with the assumption of a normal distribution: that is, if $u(x)=-e^{-r x}$, and $X \sim N\left(\mu, \sigma^{2}\right)$, then it is well-known that $-e^{-r X}$ is a lognormal distribution with mean $-e^{-r\left(\mu-\frac{r}{2} \sigma^{2}\right)}$, which clearly depends only on the mean and variance.

However, when CARA preferences are paired with a different distribution, this is no longer necessarily the case, which it should be if the preference structure is indeed driving the result. For example, consider a family of distributions $F_{Y_{p}}$ with $E\left(Y_{p}\right)=p$ and $V\left(Y_{p}\right)=V(p)$ which does not have the location-scale property. Then, the joint certainty-equivalent for a matched pair $\left(r_{1}, r_{2}\right)$ is (see Appendix 2 for the detailed derivation):

$$
\begin{aligned}
C E(p ; R) & =-R \log E\left[e^{-\frac{1}{R}\left[Y_{p}\right]}\right] \\
& =p-\frac{1}{2 R} V(p)-\sum_{n=3}^{\infty} \frac{(-1)^{n}}{n ! R^{n-1}} k_{n}\left(y_{p}\right)
\end{aligned}
$$

instead of the following, as it would be for a location-scale family of distributions:

$$
C E(p ; R)=p-\frac{1}{2 R} V(p)-\sum_{n=3}^{\infty} \frac{(-1)^{n}}{n ! R^{n-1}} V(p)^{\frac{n}{2}} k_{n}(y)
$$

The critical difference is that, with a location-scale family, each distribution $Y_{p}$ can be written as a linear function of the sequence of cumulants of the "root" random variable, $Y$. In particular, the coefficients can be expressed as a function of the variance and the risk tolerance.

This is not the case outside of the location-scale family, where each project $p$ 's returns follow a distribution described by a distinct sequence of cumulants $\left\{k_{1}(p), k_{2}(p), \ldots, k_{n}(p), \ldots\right\}$, and there is no structure on the particular relationship between the cumulants of $Y_{p}$ and $Y_{p^{\prime}}$. Hence, conditions for convexity and concavity of $C E\left(p^{*}(R) ; R\right)$ in $R$ will depend on the entire sequence $\left\{k_{1}(p), k_{2}(p), \ldots, k_{n}(p), \ldots\right\}$ for all available projects $p$, and we do not obtain the two-moment result.

\subsection{A.10: The Portfolio Choice Problem}

The model in this paper is framed to best capture the trade off between sharing a given risk versus what risk to share in the first place, and it is therefore logical to parameterize the spectrum

of risky projects with $p$, where $Y_{p}=p+V(p)^{\frac{1}{2}} Y, E(Y)=0, V(Y)=1, V^{\prime}(p)>0, V^{\prime \prime}(p)>0$. The use of $p$ and $V(p)$ makes the mean-variance trade off across projects clear.

However, one may wonder how this formulation maps into other commonly-studied portfolio choice problems, such as the classic problem of an individual who must decide how much of her 
wealth to invest in a risky versus a risk-free asset. It turns out that there is an interesting link between completeness of markets/diversification frictions and $V^{\prime \prime}(p), V^{\prime \prime \prime}(p)$. More specifically, when the market is complete and there are no frictions to diversification, $V(p)$ is quadratic and all matchings are stable (basically, CAPM). When frictions to diversification are moderate (e.g. there are moderate intermediary costs for investment), it is the case that $V^{\prime \prime \prime}(p)<0$ and PAM is the unique matching pattern. Finally, when frictions to diversification are substantial, it is the case that $V^{\prime \prime \prime}(p)>0$ and NAM is the unique matching pattern.

Suppose an individual has wealth 1 (normalized). The risky asset has returns represented by $R \sim F_{R}$, where $E(R)=\mu_{R}, V(R)=\sigma_{R}^{2}$, and the risk-free rate of return is $r_{f} \geq 0$.

Then, given that the individual invests $q \in[0,1]$ dollars in the risky asset (which can be thought of as the fraction invested in the risky asset) and $1-q$ in the risk-free asset, let the total return be described by:

$$
\tilde{R}(q)=(1-q) r_{f}+t(q) R
$$

where $0 \leq t(q) \leq q$ at each $q$, and $t^{\prime}(q)>0$. That is, it may be costly to invest: if the individual spends $q$ dollars investing in the risky asset, $q-t(q)$ dollars go to an intermediary, and $t(q)$ dollars are actually invested in the risky asset. Alternatively, instead of being a literal intermediary cost, this cost may also be interpreted as behavioral-putting aside $q$ dollars to invest may result in only $t(q)$ dollars actually being invested (e.g. for commitment reasons). If these costs are not present, then $t(q)=q$. (Of course, it may also be costly to put aside $w_{0}-q$ dollars into the risk-free asset, and this is straightforward to add to the model. However, the goal here is to show the simplest mapping of the paper's project spectrum $(p, V(p))$ where $V^{\prime \prime \prime}(p)$ may be 0 , positive, or negative, into this "risky versus risk-free asset" investment decision. For clarity, and since there are only two assets, it makes sense to "embed" the intermediary costs in one of the assets.)

Observe that the family of distributions of total return, $\{\tilde{R}(q): q \in[0,1]\}$, is a location-scale family. And:

$$
\begin{aligned}
E(\tilde{R}(q)) & =(1-q) r_{f}+t(q) \mu_{R} \\
V(\tilde{R}(q)) & =t(q)^{2} \sigma_{R}^{2}
\end{aligned}
$$

Then we can re-write:

$$
\tilde{R}(q)=\left[(1-q) r_{f}+t(q) \mu_{R}\right]+t(q) \sigma_{R} R_{0}
$$

where $R_{0}=\frac{R-\mu_{R}}{\sigma_{R}}$, so $E\left(R_{0}\right)=0, V\left(R_{0}\right)=1$.

The question is, how does this map into $Y(p)=p+V(p)^{\frac{1}{2}} Y, E(Y)=0, V(Y)=1$, where $E(Y(p))=p$ and $V(Y(p))=V(p) ?$

$$
\begin{aligned}
V(p)^{\frac{1}{2}} & \equiv t(q) \sigma_{R} \\
p & \equiv(1-q) r_{f}+t(q) \mu_{R}
\end{aligned}
$$


Rearranging yields:

$$
p-r_{f}=\frac{V(p)^{\frac{1}{2}} \mu_{R}}{\sigma_{R}}-t^{-1}\left(\frac{V(p)^{\frac{1}{2}}}{\sigma_{R}}\right) r_{f}
$$

Assume the following holds, so that there exists some positive investment amount in the risky asset which beats investing only in the risk-free asset:

$$
\begin{aligned}
p-r_{f} & >0 \Leftrightarrow \mu_{R}>\frac{t^{-1}\left(\frac{V(p)^{\frac{1}{2}}}{\sigma_{R}}\right)}{\frac{V(p)^{\frac{1}{2}}}{\sigma_{R}}} r_{f} \\
& \Leftrightarrow t(q) \mu_{R}>q r_{f} \text { for some } q
\end{aligned}
$$

For example, if $t(q)=q$, so that investing $q$ dollars in the risky asset costs exactly $q$ dollars, then this condition is simply $\mu_{R}>r_{f}$ (and investing any positive amount in the risky asset beats investing only in the risk-free asset).

First, characterize $V^{\prime}(p)$ :

$$
\begin{aligned}
1 & =\frac{\mu_{R}}{\sigma_{R}} \frac{1}{2} V(p)^{-\frac{1}{2}} V^{\prime}(p)-t^{-1 \prime}\left(\frac{V(p)^{\frac{1}{2}}}{\sigma_{R}}\right) \frac{1}{\sigma_{R}} \frac{1}{2} V(p)^{-\frac{1}{2}} V^{\prime}(p) r_{f} \Leftrightarrow \\
V^{\prime}(p) & =\frac{2 \sigma_{R}^{2} t(q)}{\mu_{R}-t^{-1 \prime}(t(q)) r_{f}}
\end{aligned}
$$

Then $V^{\prime}(p)>0$ iff:

$$
\mu_{R}>t^{-1 \prime}(t(q)) r_{f}
$$

Note that if $t(q)=q$, this just boils down to: $\mu_{R}>r_{f}$.

Second, characterize $V^{\prime \prime}(p)$ :

$$
\begin{aligned}
0= & -\frac{\mu_{R}}{\sigma_{R}} \frac{1}{4} V(p)^{-\frac{3}{2}} V^{\prime}(p)^{2}+\frac{\mu_{R}}{\sigma_{R}} \frac{1}{2} V(p)^{-\frac{1}{2}} V^{\prime \prime}(p)-t^{-1 \prime \prime}\left(\frac{V(p)^{\frac{1}{2}}}{\sigma_{R}}\right) \frac{1}{4 \sigma_{R}^{2}} V(p)^{-1} V^{\prime}(p)^{2} r_{f} \\
& +t^{-1 \prime}\left(\frac{V(p)^{\frac{1}{2}}}{\sigma_{R}}\right) \frac{1}{4 \sigma_{R}} V(p)^{-\frac{3}{2}} V^{\prime}(p)^{2} r_{f}-t^{-1 \prime}\left(\frac{V(p)^{\frac{1}{2}}}{\sigma_{R}}\right) \frac{1}{\sigma_{R}} \frac{1}{2} V(p)^{-\frac{1}{2}} V^{\prime \prime}(p) r_{f} \\
V^{\prime \prime}(p)= & \frac{\frac{\mu_{R}}{\sigma_{R}} \frac{1}{4} V(p)^{-\frac{3}{2}} V^{\prime}(p)^{2}+t^{-1 \prime \prime}\left(\frac{V(p) \frac{1}{2}}{\sigma_{R}}\right) \frac{1}{4 \sigma_{R}^{2}} V(p)^{-1} V^{\prime}(p)^{2} r_{f}-t^{-1 \prime}\left(\frac{V(p)^{\frac{1}{2}}}{\sigma_{R}}\right) \frac{1}{4 \sigma_{R}} V(p)^{-\frac{3}{2}} V^{\prime}(p)^{2} r_{f}}{\left[\frac{\mu_{R}}{2 \sigma_{R}} V(p)^{-\frac{1}{2}}-t^{-1 \prime}\left(\frac{V(p)^{\frac{1}{2}}}{\sigma_{R}}\right) \frac{1}{2 \sigma_{R}} V(p)^{-\frac{1}{2}} r_{f}\right]} \\
= & \frac{2 \sigma_{R}^{2}}{\left(\mu_{R}-t^{-1 \prime}(t(q)) r_{f}\right)^{2}}\left[1+\frac{t^{-1 \prime \prime}(t(q)) t(q)}{\left(\mu_{R}-t^{-1 \prime}(t(q)) r_{f}\right)} r_{f}\right]
\end{aligned}
$$

Then, a sufficient condition for $V^{\prime \prime}(p)>0$ is $t^{-1 \prime \prime} \geq 0\left(t^{\prime \prime} \leq 0\right)$. Clearly, $V^{\prime \prime}(p)>0$ if $t(q)=q$. 
Finally, characterize $V^{\prime \prime \prime}(p)$ :

$$
\begin{aligned}
0= & V^{\prime \prime \prime}(p)\left[\frac{\mu_{R}}{2 \sigma_{R}} V(p)^{-\frac{1}{2}}-t^{-1 \prime}\left(\frac{V(p)^{\frac{1}{2}}}{\sigma_{R}}\right) \frac{1}{2 \sigma_{R}} V(p)^{-\frac{1}{2}} r_{f}\right]+ \\
& V^{\prime \prime}(p)\left[-\frac{\mu_{R}}{4 \sigma_{R}} V(p)^{-\frac{3}{2}} V^{\prime}(p)-t^{-1 \prime \prime}\left(\frac{V(p)^{\frac{1}{2}}}{\sigma_{R}}\right) \frac{1}{4 \sigma_{R}^{2}} V(p)^{-1} V^{\prime}(p) r_{f}\right]+\frac{\mu_{R}}{\sigma_{R}} \frac{3}{8} V(p)^{-\frac{5}{2}} V^{\prime}(p)^{3} \\
& -\frac{\mu_{R}}{\sigma_{R}} \frac{1}{2} V(p)^{-\frac{3}{2}} V^{\prime}(p) V^{\prime \prime}(p)-t^{-1 \prime \prime \prime}\left(\frac{V(p)^{\frac{1}{2}}}{\sigma_{R}}\right) \frac{1}{8 \sigma_{R}^{3}} V(p)^{-\frac{3}{2}} V^{\prime}(p)^{3} r_{f} \\
& +t^{-1 \prime \prime}\left(\frac{V(p)^{\frac{1}{2}}}{\sigma_{R}}\right) \frac{1}{4 \sigma_{R}^{2}} V(p)^{-2} V^{\prime}(p)^{3} r_{f}-t^{-1 \prime \prime}\left(\frac{V(p)^{\frac{1}{2}}}{\sigma_{R}}\right) \frac{1}{2 \sigma_{R}^{2}} V(p)^{-1} V^{\prime}(p) V^{\prime \prime}(p) r_{f} \\
& +t^{-1 \prime \prime}\left(\frac{V(p)^{\frac{1}{2}}}{\sigma_{R}}\right) \frac{1}{8 \sigma_{R}^{2}} V(p)^{-2} V^{\prime}(p)^{3} r_{f}-t^{-1 \prime}\left(\frac{V(p)^{\frac{1}{2}}}{\sigma_{R}}\right) \frac{3}{8 \sigma_{R}} V(p)^{-\frac{5}{2}} V^{\prime}(p)^{3} r_{f} \\
& +t^{-1 \prime}\left(\frac{V(p)^{\frac{1}{2}}}{\sigma_{R}}\right) \frac{1}{2 \sigma_{R}} V(p)^{-\frac{3}{2}} V^{\prime}(p) V^{\prime \prime}(p) r_{f}
\end{aligned}
$$

Simplify:

$$
\begin{aligned}
0 & =V^{\prime \prime \prime}(p) \frac{1}{V^{\prime}(p)}-\frac{3 V^{\prime \prime}(p)^{2}}{V^{\prime}(p)^{2}}+\frac{V^{\prime \prime}(p)}{V(p)}+\frac{\mu_{R}}{4 \sigma_{R}} \frac{V^{\prime}(p) V^{\prime \prime}(p)}{V(p)^{\frac{3}{2}}}-t^{-1 \prime \prime \prime}\left(\frac{V(p)^{\frac{1}{2}}}{\sigma_{R}}\right) \frac{1}{8 \sigma_{R}^{3}} V(p)^{-\frac{3}{2}} V^{\prime}(p)^{3} r_{f} \\
V^{\prime \prime \prime}(p) & =\left[V^{\prime \prime}(p)\left[\frac{3 V^{\prime \prime}(p)}{V^{\prime}(p)^{2}}-\frac{1}{V(p)}-\frac{\mu_{R}}{4 \sigma_{R}} \frac{V^{\prime}(p)}{V(p)^{\frac{3}{2}}}\right]+t^{-1 \prime \prime \prime}\left(\frac{V(p)^{\frac{1}{2}}}{\sigma_{R}}\right) \frac{1}{8 \sigma_{R}^{3}} V(p)^{-\frac{3}{2}} V^{\prime}(p)^{3} r_{f}\right] V^{\prime}(p)
\end{aligned}
$$

Use the known expressions for $V^{\prime}(p)$ and $V^{\prime \prime}(p)$ :

$$
\begin{aligned}
\frac{V^{\prime \prime \prime}(p)}{V^{\prime}(p)} & =\frac{2 \sigma_{R}^{2}}{\left(\mu_{R}-t^{-1 \prime}(t(q)) r_{f}\right)^{2}}\left[1+\frac{t^{-1 \prime \prime}(t(q)) t(q)}{\left(\mu_{R}-t^{-1 \prime}(t(q)) r_{f}\right)} r_{f}\right]\left[\frac{1}{2 t(q)^{2} \sigma_{R}^{2}}+\frac{3 t^{-1 \prime \prime}(t(q)) t(q) r_{f}-\left(\mu_{R}-r_{f}\right)}{2 \sigma_{R}^{2} t(q)^{2}\left(\mu_{R}-t^{-1 \prime}(t(q)) r_{f}\right)}\right]+ \\
& +\frac{t^{-1 \prime \prime \prime}(t(q))}{\left(\mu_{R}-t^{-1 \prime}(t(q)) r_{f}\right)^{3}} r_{f}
\end{aligned}
$$

Thus, we see that:

1. When $t(q)=q$ (the "standard case"), it's clear that $V^{\prime}(p)>0, V^{\prime \prime}(p)>0$, and $V^{\prime \prime \prime}(p)=0$, as long as $\mu_{R}>r_{f}$ (which must hold in order for any positive investment in the risky asset to be preferred to investing solely in the risk-free asset). Hence, this fits neatly into the framework of the paper, and by the main result, all matching patterns are stable in this case (since $\left.V^{\prime \prime \prime}(p)=0\right)$.

2. When $0 \leq t(q) \leq q, t(q) \neq q, t^{\prime}(q)>0$ (so that there are positive intermediary costs associated with investment), then as long as investing some positive amount in the risky asset beats investing solely in the risky asset $\left(\mu_{R}>t^{-1 \prime}(t(q)) r_{f}\right)$, it is the case that $V^{\prime}(p)>0$. 
Moreover, if $t^{\prime \prime}(q)<0$, so that the amount actually invested in the risky asset is increasing with the amount spent on investing, but at a decreasing rate, then $V^{\prime \prime}(p)>0$ holds. Finally:

(a) As $t^{\prime \prime \prime}(q)$ becomes more negative $\left(t^{-1 \prime \prime \prime}>0\right), V^{\prime \prime \prime}(p)$ becomes positive: the more severe the intermediary costs $(t(q)$ is "very concave"), the larger the increase in variance associated with an increase in mean return. This is the case where $N A M$ is the unique matching pattern $\left(V^{\prime \prime \prime}(p)>0\right)$.

(b) As $t^{\prime \prime \prime}(q)$ becomes more positive $\left(t^{-1 \prime \prime \prime}<0\right), V^{\prime \prime \prime}(p)$ becomes negative: the less severe the intermediary costs $(t(q)$ is "not so concave"), the increase in variance associated with an increase in mean return increases less sharply. This is the case where $P A M$ is the unique matching pattern $\left(V^{\prime \prime \prime}(p)<0\right)$.

This somewhat stylized mapping suggests an interesting link between different curvatures of $V(p)$ and market completeness: when the market is complete and there are no constraints on diversification (the case $t(q)=q$ ), it is the case that $V^{\prime \prime \prime}(p)=0$ and all matchings are stable. When the market is "somewhat incomplete" / there are weak constraints on diversification, that is, $t(q)<q$ and $t^{\prime \prime}(q)<0$, but $t^{\prime \prime \prime}(q)>0$, so that intermediary costs do not increase too sharply as investment increases, it is the case that $V^{\prime \prime \prime}(p)<0$ and $P A M$ is the unique matching pattern. Finally, when the market is "very incomplete"/there are strong constraints on diversification, that is, $t(q)<q$ and $t^{\prime \prime}(q)<0$, but $t^{\prime \prime \prime}(q)<0$, so that intermediary costs increase sharply as investment increases, then it is the case that $V^{\prime \prime \prime}(p)>0$ and $N A M$ is the unique matching pattern.

\subsection{A.11: Individual Payoffs}

Under what more general conditions are the more risk-averse agents worse off after a policy like the one studied in Section 4 ?

Since this is meant to give an idea of the conditions, rather than to be an exhaustive proof, I focus on the "best case scenario for the most-risk averse agent" case. That is, I ask under which conditions the most risk-averse agent's highest possible equilibrium payoff pre-policy is higher than the most risk-averse agent's highest possible equilibrium payoff post-policy. Recall that this implies that the most risk-averse agent receives $v_{4}^{\text {pre }}=C E_{14}^{\text {pre }}$ pre-policy, when the unique matching is negativeassortative, and $v_{4}^{\text {post }}=C E_{44}^{\text {post }}$ post-policy, when the unique matching is positive-assortative (see Section 4).

Then, the question is, when do the following hold?

$$
\begin{aligned}
& u_{G 2,1}^{\text {pre }}>u_{G 2,1}^{\text {post }} \Leftrightarrow \\
& \left(C E_{31}^{N}-C E_{32}^{N}\right)+\left(C E_{22}^{N}-C E_{23}^{N}\right)+\left(C E_{13}^{N}-C E_{14}^{N}\right)+C E_{14}^{N}> \\
& \left(C E_{21}^{\text {post }}-C E_{22}^{\text {post }}\right)+\left(C E_{32}^{\text {post }}-C E_{33}^{\text {post }}\right)+\left(C E_{43}^{\text {post }}-C E_{44}^{\text {post }}\right)+C E_{44}^{P}
\end{aligned}
$$




$$
\begin{aligned}
u_{G 2,2}^{\text {pre }} & >u_{G 2,2}^{\text {post }} \Leftrightarrow \\
\left(C E_{22}^{N}-C E_{23}^{N}\right)+\left(C E_{13}^{N}-C E_{14}^{N}\right)+C E_{14}^{N}>\left(C E_{32}^{\text {post }}-C E_{33}^{\text {post }}\right)+\left(C E_{43}^{\text {post }}-C E_{44}^{\text {post }}\right)+C E_{44}^{P} & \\
\left(C E_{13}^{N}-C E_{14}^{N}\right)+C E_{14}^{N} & >\left(C E_{43}^{\text {post }}-C E_{44}^{\text {post }}\right)+C E_{44}^{P} \\
u_{G 2,3}^{\text {pre }} & >u_{G 2,}^{\text {post }} \Leftrightarrow \\
& \\
u_{G 2,4}^{\text {pre }} & >u_{G 2,4}^{\text {oost }} \Leftrightarrow \\
C E_{14}^{N} & >C E_{44}^{P}
\end{aligned}
$$

Reducing these conditions shows that the more risk-averse agents are made worse off by a policy which moves the spectrum of portfolio choices from $M^{\prime \prime}(p)=\varepsilon$ to $M^{\prime \prime}(p)=-\varepsilon$-that is, a policy which moves the spectrum of portfolio choices from negative-assortative matching just above the "indifference point" (where all matches are stable), to positive-assortative matching just below. This is exactly the kind of policy studied in Section 4: a policy which results more in a change in the risk trade-off across different portfolios than in risk levels.

Why is this the case? Again, focus on the case of normally-distributed returns $Y_{p} \sim N\left(p, p^{N}\right)$ from Section 4 to simplify the form of the joint certainty-equivalents. Then, the "indifference point" is at $N=2$ : if $Y_{p} \sim N\left(p, p^{2}\right)$, all matchings are stable, and:

$$
C E(x, y)-C E\left(x, y^{\prime}\right)=C E\left(x^{\prime}, y\right)-C E\left(x^{\prime}, y^{\prime}\right) \forall x, y, x^{\prime}, y^{\prime}
$$

Thus, for $N \in[2-\varepsilon, 2+\varepsilon]$ :

$$
C E(x, y)-C E\left(x, y^{\prime}\right) \approx C E\left(x^{\prime}, y\right)-C E\left(x^{\prime}, y^{\prime}\right) \forall x, y, x^{\prime}, y^{\prime}
$$

and the four conditions above effectively reduce to:

$$
C E_{14}^{N}>C E_{44}^{P}
$$

which holds, since $C E^{N} \approx C E^{P}$, and the joint certainty-equivalent is decreasing in risk aversion.

\subsection{A.12: Limited commitment}

While I leave a rigorous treatment of limited commitment in this setting to future work, I discuss some preliminary analysis here.

The basic elements of the two-period model with limited commitment are: 
1. At the beginning of each period, each member of a matched group (or as an individual, if in autarky) chooses a risky project.

(a) Assume that eternal autarky is the punishment for reneging, and that incomes are independent across time and people, since we know that when incomes are independent, autarky is never optimal. Thus, imposing autarky is in fact a punishment.

2. Basic timing of the game:

(a) A matched pair agrees to a return-contingent sharing rule for their pooled incomes at the beginning of pd. 1 .

(b) Each individual in a matched pair observes both income realizations, and so knows what the transfer specified by the rule is. Each can choose to uphold that transfer, or to renege (do something other than what the rule specifies). If at least one member reneges, then both members are consigned to autarky in pd. 2. (Assume no re-matching.)

(c) If both uphold the transfer, then the pair stays matched in pd. 2. Assume that in pd. 2 , pairs that successfully cooperated in pd. 1 can implement the efficient outcome in pd. 2 (for example, because they've successfully won each other's trust).

Then, what do we know about the equilibrium?

In pd. 2, for non-reneging pairs, we know that NAM conditions imply that the less risk-averse differentially prefer to bid more for the more risk-averse, while PAM conditions imply that the less risk-averse differentially prefer to bid more for the less risk-averse.

So, under NAM conditions, a less risk-averse person finds autarky in pd. 2 more costly with a more risk-averse partner than with a less risk-averse partner. Under PAM, a less risk-averse person finds autarky in pd. 2 more costly with a less risk-averse partner than a more risk-averse partner.

So far, imperfect commitment hasn't changed when PAM is optimal versus when NAM is optimal.

Now, think about the effect on pd. 1 choices. Because of limited commitment, in pd. 1, a matched pair can't always implement the efficient risk-sharing rule. The equilibrium sharing rule in pd. 1 for a matched pair $\left(r_{i}, r_{j}\right)$ is instead:

1. If the income realizations are such that the efficient risk-sharing rule happens to be such that both partners find $U$ (keep my own income realization) $-U\left(\frac{r_{j}}{r_{i}+r_{j}} Y_{p(i, j)}\right)<U\left(\frac{r_{j}}{r_{i}+r_{j}} Y_{p_{-} o p t i m a l}\right)-$ $U($ aut $)$, then the transfer is the efficient one.

2. If the realizations are outside of this interval (which will loosely be the case when one partner has a particularly good realization and the other has a particularly bad one), then the better off partner should make a transfer to her partner that makes her indifferent between paying that transfer and keeping everything and facing autarky in pd. 2. (Note that if reneging is better than cooperating, the optimal reneging is to keep your own realization.) 
Note that this rule is much less smooth than the efficient risk-sharing rule, which is a linear split of pooled output. Thus, introducing limited commitment means that the equilibrium split of pooled income is linear for some joint realizations (that is, realizations which are similar), and near-autarky for others (very dissimilar realizations).

More risk-averse people find autarky worse, and also dislike the volatility of the sharing rule in pd. 1 under limited commitment. So even though under NAM conditions, a more risk-averse person prefers a less risk-averse partner in pd. 2, the volatility of their corresponding pd. 1 sharing rule (and the fact that the less risk-averse person finds autarky a lesser threat than the more risk-averse person), means that more risk-averse people prefer to match with other more risk-averse people more strongly than they do in the absence of limited commitment. On the other hand, the less risk-averse especially value a more risk-averse partner, because a more risk-averse partner is willing to receive a small transfer and is also the best partner in pd. 2 (NAM conditions). However, limited commitment constrains the credible "bid" a less risk-averse partner can make in pd. 1. That is, a less risk-averse person would like to promise a more risk-averse person more than the transfer that makes her indifferent between honoring the transfer and keeping her own income and facing autarky in pd. 2, but she can't do this credibly. Thus, under limited commitment, we would expect positive-assortative matching to be more likely than when commitment is perfect.

\subsection{A.13: Background and Empirical Context}

The purpose of this section is to provide further empirical context for the model. First, I will discuss the substantial role played by informal insurance motivations in building relationships in risky environments with missing formal insurance and credit markets. Additionally, I will show that risk attitudes are a significant determinant of risk-sharing partner choice.

Next, I will provide evidence that there is a great deal of heterogeneity in risk aversion across individuals in a wide range of settings.

Finally, I will provide evidence of heterogeneity in the riskiness of activities available to individuals, as well as heterogeneity in the relative riskiness of these activities across different environments.

To fix ideas, it may be helpful to envision an agricultural setting, which captures nicely the key elements of the model. Much of the literature discussed in this section is drawn from an agricultural context, where landowners and farmers are heterogeneous in the extent of their risk aversion, and landowners must decide which farmers to work with. Different crops have different yield and profit distributions: some crops are very robust to drought but correspondingly tend to produce low yields on average ("safe" crops), while other crops have the potential for very high yields, but are extremely sensitive to rainfall and other inputs, and blight easily ("risky" crops). In addition to crop portfolio and plot locations, fertilizer and other inputs, irrigation, planting times, and general farming methods and technologies must also be chosen.

Furthermore, the yield and profit distribution of each crop varies across agroclimactic region. Different parts of the world experience different levels of rainfall, soil quality, irrigation, elevation, heat, and other such ecological characteristics, and this influences the stochastic yield and profit of 
each crop. It is no surprise, then, that equilibrium cropping methods, crop mixes, and contracting institutions vary so widely across region. A goal of this paper is to advance the understanding of these differences.

\subsubsection{Risk Attitude and Informal Insurance Relationships}

An abundance of work discusses the considerable role of informal insurance concerns in network formation. People rely on each other to smooth consumption risk and income risk in a wide variety of ways (Alderman and Paxson (1992), Morduch (1995)). A very prevalent consumption-smoothing technique between people is transfers and remittances, and much work has been done to study the nature of the transfers that can be sustained given a risk-sharing group, the shapes of equilibrium networks holding fixed some transfer rule, and who is empirically observed to make transfers with whom (Townsend (1994), Fafchamps and Lund (2003), Genicot and Ray (2003), Bramoulle and Kranton (2007), and Ambrus et al. (2013), to name a few). A general message these papers convey is that the need to manage risk in the absence of formal insurance institutions has huge effects on interpersonal relationships among the poor.

In fact, risk management can affect relationship formation in very specific ways. Rosenzweig and Stark (1989) show that daughters are often strategically married to villages located in environmentally dissimilar regions with minimally correlated farming incomes, for the purposes of consumption-smoothing; households exposed to more income risk are more likely to invest in longerdistance marriage arrangements. Ligon et al. (2002), Fafchamps (1999), and many others analyze a pure risk-sharing relationship between two heterogeneously risk-averse households who perfectly observe each other's income. Ackerberg and Botticini (2002) study agricultural contracting in medieval Tuscany, and find evidence that heterogeneously risk-averse tenant farmers and landlords strategically formed sharecropping relationships based on differing risk attitudes, motivated by risk management concerns. Hence, informal insurance motivations play a substantial role in the formation of relationships.

But how much do individuals care about the risk attitudes of potential partners when forming risk-sharing groups? Naturally, there are many other reasons people might match with each other, but the point of the model is to focus on one important determinant of risk-sharing relationship formation, and to study how equilibrium matching patterns shift along that dimension. Further-

more, there is a great deal of evidence that the risk attitudes of partners are indeed a primary determinant of risk-sharing partner choice. Ackerberg and Botticini (2002) provide empirical evidence supporting the presence of endogeneity of matching along risk attitude of landowners and sharecroppers in medieval Tuscany. In their data, they find that share contracts were associated with the safer crop of cereal, while fixed rent (residual claimancy) contracts were associated with the riskier crop of vines. They argue that this is the outcome of endogenous matching-risk-neutral tenants may have been assigned to the riskier crops, resulting in fixed rent contracts for vines, while risk-averse tenants may have been assigned to the safer crops, resulting in share contracts on cereals. 
Additional evidence for the importance of risk attitudes as a determinant of risk-sharing relationships is found in Gine et al. (2010) and Attanasio et al. (2012). Gine et al. (2010) run an experiment on small-scale entrepreneurs in urban Peru and allow joint liability groups to form endogenously in a microfinance setting. They find strong evidence of assortative matching along risk attitude. Attanasio et al. (2012) run a unique experiment with 70 Colombian communities. They gather data about risk attitudes and pre-existing kinship/friendship networks, and then allow individuals to form risk-sharing groups of any size. Attanasio et al. find that, when members know each other's risk types, and trust each other (family and friends are in the same group), conditioning on all other potential reasons for matching which they are able to account for (gender, age, geography), there is strong evidence of positive assortative matching along risk aversion.

To further emphasize the significant role of risk attitude in determining risk-sharing relationship formation, I use the dataset from Attanasio et al. (2010) to calculate the proportion of formed links that involved at least one family or one friendship tie, for each municipality. The mean of these proportions is 0.005 , or $0.5 \%$. Since it's possible that there were very few family and friend ties reported in the entire dataset to begin with, I also calculate the proportion of all possible links that could have involved at least one family or friendship tie, for each municipality. The mean of this number is 0.05 . Hence, this back-of-the-envelope calculation suggests that, in this setting, only about $10 \%$ of all possible risk-sharing relationships which could have involved a family or friendship tie, actually did involve such a tie. Hence, while one might expect kinship and friendship to be major influences in partner choice, there is strong evidence that risk attitude is the more prominent consideration when the partner is being chosen specifically for the purposes of informal insurance. In particular, the family and friendship tie is likely to influence the pool of potential partners (because individuals are less likely to know the risk attitudes of strangers, or to trust them), but the choice of partner from this pool for the purposes of insurance is primarily determined by risk attitudes.

\subsubsection{Heterogeneity in Risk Aversion}

The second key piece of the model is heterogeneity in risk attitudes across individuals. There is plenty of evidence that people are risk-averse and that they are heterogeneous in their risk-aversion. Experiments which elicit risk attitudes by asking subjects to choose from a set of gambles differing in riskiness find much variation in gamble choice. For example, Harrison et al. (2010) asked 531 experimental subjects drawn from India, Ethiopia, and Uganda to choose a gamble from a set of gambles varying in riskiness (a riskier gamble has higher mean but correspondingly higher variance), in a similar spirit as the seminal study by Binswanger (1980), and estimated the density of CRRA risk attitudes: 


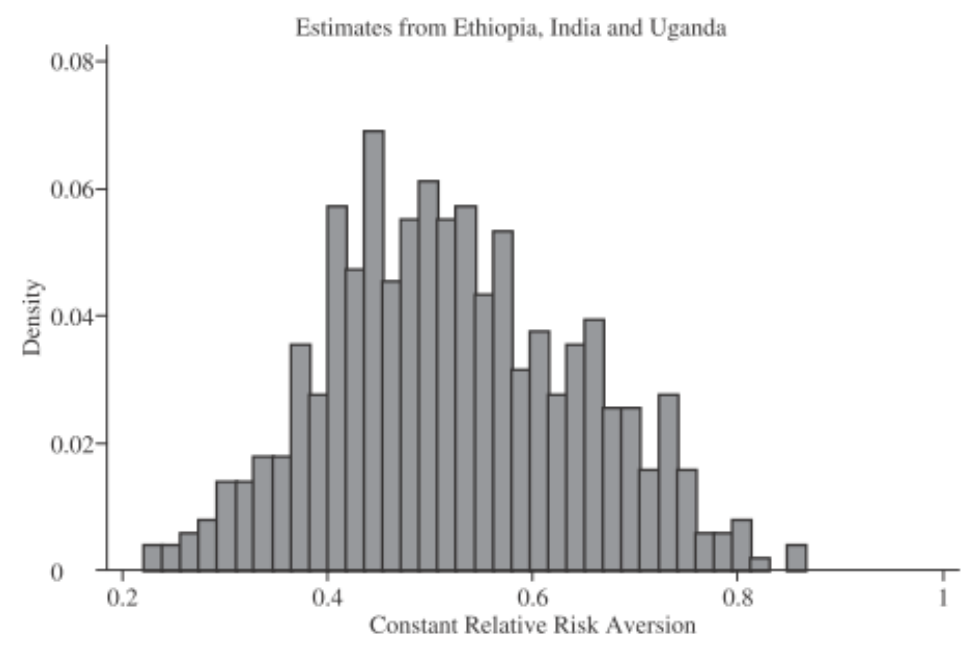

It's clear that there is a substantial amount of variation, and almost every point in the $[0,1]$ range is represented.

In another experiment involving over 2,000 people living in 70 Colombian communities, where $66 \%$ live in rural areas, Attanasio et al. (2012) observes the following distribution (gamble 1 is the safest gamble, while gamble 6 is the riskiest):

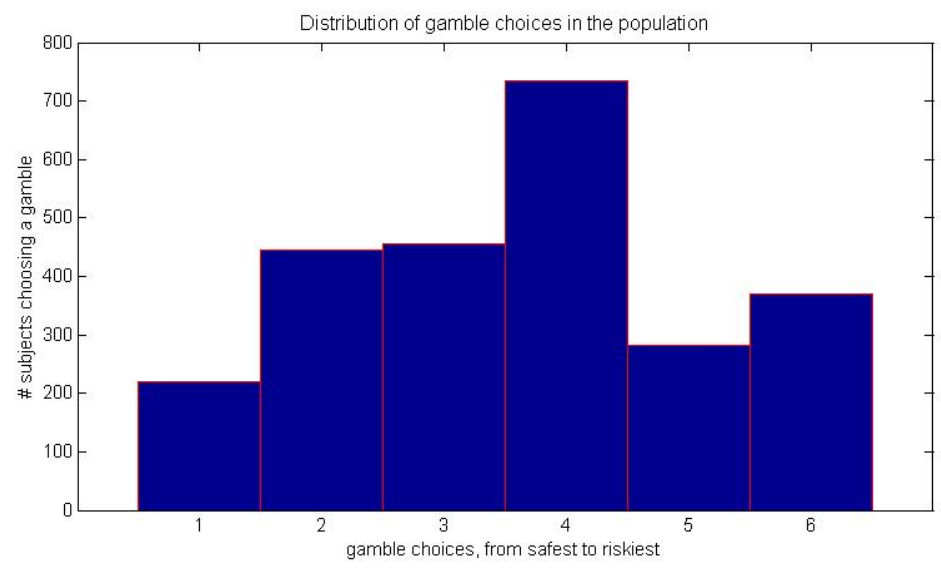

Chiappori et al. (2010) use two distinct methods to measure heterogeneity in risk preferences in Thai villages, where these villages are spread across several regions in Thailand. The first method is based off the co-movement of individual consumption with aggregate consumption, and the second is based off of optimal portfolio choice theory. Using both methods, they find substantial heterogeneity in risk attitudes in each village. Moreover, this heterogeneity varies across villages and regions. The following table reports the means of risk tolerance for each of 16 villages, and the test statistic for heterogeneity: 


\begin{tabular}{|c|c|c|c|c|c|c|c|}
\hline \multirow[b]{2}{*}{ village } & \multirow[b]{2}{*}{ households } & \multicolumn{3}{|c|}{ risk aversion $\gamma_{i}$} & \multicolumn{3}{|c|}{ risk tolerance $1 / \gamma_{i}$} \\
\hline & & mean & $\chi^{2}$ & p-value & mean & $\chi^{2}$ & p-value \\
\hline \multicolumn{8}{|c|}{ Chachoengsao } \\
\hline 2 & 13 & 2.00 & 277.29 & 0.0000 & 1.56 & 3543.60 & 0.0000 \\
\hline 4 & 21 & 0.79 & 78.44 & 0.0000 & 2.47 & 1646.42 & 0.0000 \\
\hline 7 & 6 & 0.98 & 6.69 & 0.3509 & 1.28 & 32.21 & 0.0000 \\
\hline 8 & 14 & 0.61 & 31.11 & 0.0053 & 5.11 & 7986.64 & 0.0000 \\
\hline \multicolumn{8}{|c|}{ Buriram } \\
\hline 2 & 18 & 0.62 & 12.54 & 0.8184 & 2.97 & 368.59 & 0.0000 \\
\hline 10 & 8 & 0.34 & 5.87 & 0.6618 & 4.02 & 147.64 & 0.0000 \\
\hline 13 & 10 & 0.41 & 14.27 & 0.1610 & 7.61 & 2255.00 & 0.0000 \\
\hline 14 & 15 & 0.84 & 73.55 & 0.0000 & 3.55 & 4209.49 & 0.0000 \\
\hline \multicolumn{8}{|c|}{ Lop Buri } \\
\hline 1 & 19 & 1.20 & 96.08 & 0.0000 & 1.36 & 1011.17 & 0.0000 \\
\hline 3 & 8 & 2.12 & 348.07 & 0.0000 & 1.33 & 3981.73 & 0.0000 \\
\hline 4 & 27 & 1.40 & 173.59 & 0.0000 & 1.29 & 2061.54 & 0.0000 \\
\hline 6 & 24 & 1.82 & 485.27 & 0.0000 & 1.29 & 3074.97 & 0.0000 \\
\hline \multicolumn{8}{|c|}{ Sisaket } \\
\hline 1 & 22 & 0.43 & 21.94 & 0.4633 & 3.78 & 457.10 & 0.0000 \\
\hline 6 & 34 & 0.78 & 117.07 & 0.0000 & 1.85 & 2010.67 & 0.0000 \\
\hline 9 & 22 & 0.76 & 33.96 & 0.0495 & 3.24 & 2141.48 & 0.0000 \\
\hline 10 & 13 & 0.47 & 9.68 & 0.7199 & 2.90 & 36.03 & 0.0006 \\
\hline \multicolumn{8}{|c|}{ pooled } \\
\hline- & 274 & 0.98 & 1358.43 & 0.0000 & 2.64 & 77568.89 & 0.0000 \\
\hline
\end{tabular}

Again, it is clear that there is widespread variation in the degree of risk aversion across households.

\subsubsection{Heterogeneity in Risky Activities and Settings}

Finally, agents in a given setting have a wide variety of investment options and household decisions to make, which vary in riskiness. For example, a farmer must choose a spatial distribution of his plots, what lumpy purchases to make (e.g. bullocks), and when and how to plant his crop. A microentrepreneur must decide what kind of business he wants to start. Parents must decide how to invest household resources, and whom their children will marry. Individuals face a diversity of choices, and how much diversity, as well as the relative riskiness of one decision compared to another, varies across settings.

For example, Rosenzweig and Binswanger (1993) consider the equilibrium crop portfolio choices of heterogeneously risk-averse farmers living in six ICRISAT villages located across three distinct agroclimactic regions in India. The first region is characterized by low levels of erratically distributed rainfall and soils with limited water storage capacity (this is the riskiest environment), the second region by similarly erratic rainfall and irrigation but better soil storage capacity, and the third region by low levels of more reliable rainfall with reasonable soil storage capacity (this is the safest environment). The principal crops grown are sorghum, pigeon peas, pearl millet, chickpeas, and groundnuts, and their yield distributions vary across environment. They show that differences 
in risk aversion do translate into differences in choice of risky investments. Individuals are influenced by risk-reduction when choosing income streams, particularly in response to limitations on ex post consumption-smoothing, and the degree to which they are influenced depends on their risk aversion.

Dercon (1996) also studies the variation in riskiness of agricultural investment decisions by heterogeneously risk-averse rural households. His data is drawn from Tanzania, a country with very underdeveloped credit markets (in 1989, only $5 \%$ of commercial bank lending went to the private sector, and less than $10 \%$ of this lending went to individual farmers). The UN Food and Agriculture Organization provides an interesting look at the vast heterogeneity in crop yield distributions and equilibrium crop mix across regions in Tanzania in 1998. The following table shows the area, yield, and production of each of five crops across ten agroclimactically heterogeneous regions in Tanzania $^{22}$ :

Table 2: Tanzania - 1998 Vuli Crop Production in Cereal Equivalent by Region

\begin{tabular}{|c|c|c|c|c|c|c|c|c|c|c|c|c|c|c|c|}
\hline \multirow[b]{2}{*}{ Region } & \multicolumn{3}{|c|}{ Maize } & \multicolumn{3}{|c|}{ Sorghum } & \multicolumn{3}{|c|}{ Paddy } & \multicolumn{3}{|c|}{ Millet } & \multicolumn{3}{|c|}{ Wheat } \\
\hline & \begin{tabular}{|c} 
Area \\
(0000 \\
ha)
\end{tabular} & \begin{tabular}{|c} 
Yield \\
(kg/ha)
\end{tabular} & \begin{tabular}{|c|} 
Production \\
(tonnes)
\end{tabular} & $\begin{array}{c}\text { Area } \\
\text { (c000 } \\
\text { ha) }\end{array}$ & $\begin{array}{c}\text { Yield } \\
\text { (kg/ha) }\end{array}$ & \begin{tabular}{|c|} 
Production \\
(tonnes)
\end{tabular} & $\begin{array}{c}\text { Area } \\
\text { (c000 } \\
\text { ha) }\end{array}$ & \begin{tabular}{|c|} 
Yield \\
$(\mathrm{kg} / \mathrm{ha})$
\end{tabular} & $\begin{array}{c}\text { Production } \\
\text { (tonnes) }\end{array}$ & $\begin{array}{c}\text { Area } \\
\text { (000 } \\
\text { ha) }\end{array}$ & $\begin{array}{c}\text { Yield } \\
(\mathrm{kg} / \mathrm{ha})\end{array}$ & $\begin{array}{l}\text { Production } \\
\text { (tonnes) }\end{array}$ & $\begin{array}{c}\text { Area } \\
\text { (c000 } \\
\text { ha) }\end{array}$ & \begin{tabular}{|c|} 
Yield \\
$(\mathrm{kg} / \mathrm{ha})$
\end{tabular} & $\begin{array}{c}\text { Production } \\
\text { (tonnes) }\end{array}$ \\
\hline Mara & 13.0 & 1700 & 22.1 & 20.0 & 1300 & 26.0 & 1.0 & 1600 & 1.6 & 0.5 & 1100 & 0.6 & 0.0 & 0 & 0.0 \\
\hline Arusha & 50.0 & 1600 & 80.0 & 10.0 & 1200 & 12.0 & 2.6 & 2300 & 5.98 & 3.4 & 1000 & 3.4 & 14.0 & 1700 & 23.8 \\
\hline Kilimanjaro & \begin{tabular}{|l|}
30.0 \\
\end{tabular} & 1500 & 45.0 & 1.5 & 1300 & 2.0 & 3.2 & 2500 & 8 & - & - & - & 1.2 & 1400 & 1.7 \\
\hline Tanga & 20.0 & 1500 & 30.0 & - & - & - & 2.1 & 1900 & 3.99 & - & - & - & 0.7 & 1000 & 0.7 \\
\hline Morogoro & 40.0 & 1400 & 56.0 & 8.0 & 1200 & 9.6 & 0.0 & 0.0 & 0.0 & 0.0 & 0.0 & 0.0 & 0.0 & 0.0 & 0.0 \\
\hline Mbeya & 20.0 & 1600 & 32.0 & 0.0 & 0.0 & 0.0 & 1.0 & 1800 & 1.8 & 0.0 & 0.0 & 0.0 & 0.0 & 0.0 & 0.0 \\
\hline Coast/DSM & \begin{tabular}{|l|}
$\mid 11.0$ \\
\end{tabular} & 1100 & 12.1 & - & - & - & - & - & - & - & - & - & - & - & - \\
\hline Kagera & 51.0 & 1500 & 76.5 & 2.7 & 1300 & 3.5 & 0.1 & 2000 & 0.2 & - & - & - & - & - & - \\
\hline Kigoma & 40,0 & 1800 & 72.0 & 3.0 & 1500 & 4.5 & 3,0 & 2000 & 6 & 0.1 & 1000 & 0.1 & - & - & - \\
\hline Mwanza & 100.0 & 1200 & 120.0 & 110.0 & 1100 & 121.0 & 10.0 & 1800 & 18 & 1.0 & 1000 & 1.0 & & & \\
\hline Total & 375 & & 545.7 & 155.2 & & 178.6 & 23.0 & & 45.6 & 5.0 & & 5.1 & 15.9 & & 26.2 \\
\hline
\end{tabular}

Unfortunately, this table excludes estimates of the variance of yield of each of these crops across regions. Dercon (1996) provides a discussion of this in his paper. He describes a multiplicity of soils and irrigation systems in Tanzania, which support different crops. Paddy, a crop which can yield a high return, is restricted only to specific soils and areas close to a river, and is the least drought and locust resistant. Despite the potential for high returns, only $11 \%$ of the total cultivation sample grew paddy. On the other hand, sorghum yields only a low-moderate return, but all soils can sustain it, and it is more resistant to drought and pests. Even though it had a lower mean return, it was grown by all but two households in the sample.

Uganda and Ethiopia are similar to Tanzania in the set of crops grown, though the actual crop mix grown differs due to differences in environmental conditions. An IFPRI report from 2011 estimating crop yields in Uganda provides a useful illustration of how the variance of crop yields differs across crops, and the typical relationship of the variance with the mean:

\footnotetext{
${ }^{22}$ Of course, in addition to levels and fluctuations of crop yields, farmers care about the levels and fluctuations of crop prices, as they care ultimately about the distribution of profits.
} 
Figure A.1-Average yield and yield range of selected unfertilized crops observed in on-farm surveys (farmer recall) in Uganda, by variety, type, and average of annual national yield estimates (1970-2000)

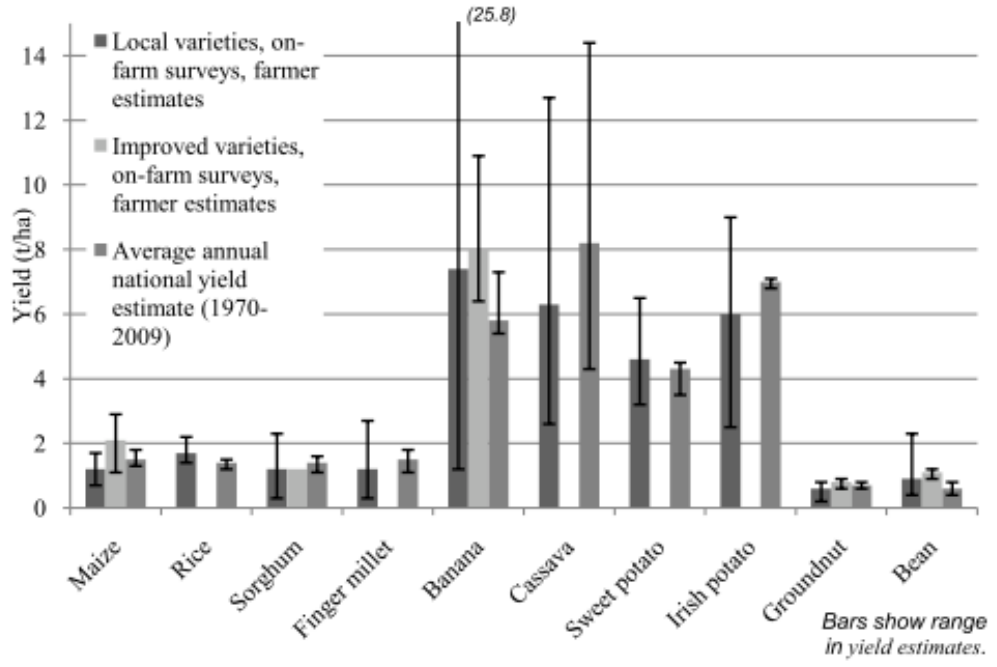

Source: Compiled by authors based on database of crop yields in Uganda.

There is a clear positive relationship between mean yield and variance of yield. Groundnuts have low mean yields and correspondingly low fluctuation of yields, making it a "safer" crop, while banana has much higher mean yields but correspondingly higher fluctuation of yields, making it a "riskier" crop.

Abebe et al. (2010) provide a similar graphic for Ethiopia:

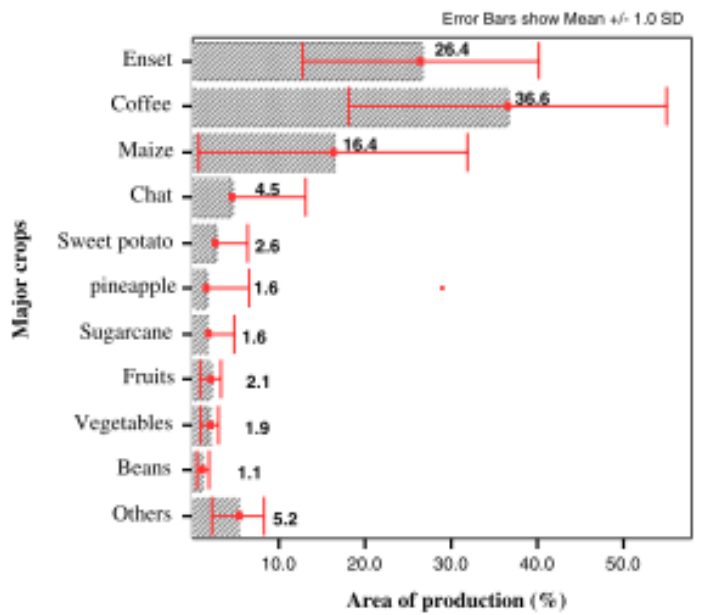

Fig. 3 Mean area share (in percentage of the farm area) of the major crops in farms. All 144 farms are used here. Error bars indicate one standard deviation

(Enset is a type of banana.)

Again, we get a general sense that higher mean yield crops have a higher variance of yield, while lower mean yield crops have a smaller variance of yield. Comparing across Uganda and Ethiopia, 
we see that maize is a safer crop relative to sweet potato in Uganda, but the opposite is true in Ethiopia. Thus, the same set of crops have very different yield distributions in different settings, and furthermore, each crop's relative riskiness with the other crops also varies across setting. 